\title{
Miniaturized Interferometric Sensors with Spectral Tunability for Optical Fiber Technology-A Comparison of Size Requirements, Performance, and New Concepts
}

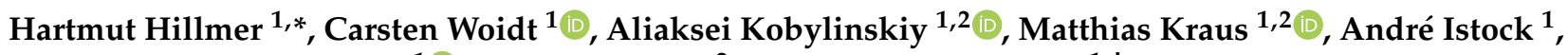 \\ Mustaqim S. Q. Iskhandar ${ }^{1} \mathbb{D}$, Robert Brunner ${ }^{2}$ and Thomas Kusserow ${ }^{1,+}$ \\ 1 Institute of Nanostructure Technologies and Analytics (INA) and Center for Interdisciplinary Nanostructure \\ Science and Technology (CINSaT), University of Kassel, 34132 Kassel, Germany; \\ woidt@ina.uni-kassel.de (C.W.); Aliaksei.Kobylinskiy@eah-jena.de (A.K.); Matthias.Kraus@eah-jena.de (M.K.); \\ istock@ina.uni-kassel.de (A.I.); iskhandar@uni-kassel.de (M.S.Q.I.); kusserow@ina.uni-kassel.de (T.K.) \\ 2 Applied Optics, Department SciTec, University of Applied Sciences Jena, Carl-Zeiss-Promenade 2, \\ 07745 Jena, Germany; Robert.Brunner@eah-jena.de \\ * Correspondence: hillmer@ina.uni-kassel.de \\ + Present address: Institute of Microsystems Technology, Hamburg University of Technology, \\ Eißendorferstr. 42, 21073 Hamburg, Germany.
}

check for updates

Citation: Hillmer, H.; Woidt, C.; Kobylinskiy, A.; Kraus, M.; Istock, A.; Iskhandar, M.S.Q.; Brunner, R.;

Kusserow, T. Miniaturized

Interferometric Sensors with Spectral Tunability for Optical Fiber Technology-A Comparison of Size Requirements, Performance, and

New Concepts. Photonics 2021, 8, 332. https: / / doi.org/10.3390/

photonics 8080332

Received: 28 June 2021

Accepted: 26 July 2021

Published: 13 August 2021

Publisher's Note: MDPI stays neutral with regard to jurisdictional claims in published maps and institutional affiliations.

Copyright: (c) 2021 by the authors. Licensee MDPI, Basel, Switzerland. This article is an open access article distributed under the terms and conditions of the Creative Commons Attribution (CC BY) license (https:// creativecommons.org/licenses/by/ $4.0 /)$.

\begin{abstract}
Optical interferometric sensors have acquired significant importance in metrology and information technology, especially in terms of their potential application in launching size, selectivity, sensitivity, resolution, spectral tuning ranges, efficiency, and cost. However, these demands are often contradictory and counteract one another, and are thus difficult to simultaneously fulfill during their interaction. This review focuses on a detailed comparison of seven different strongly miniaturized sensor concepts investigating the limits of these demands. For the visible and near-infrared spectral range, seven optical sensors were reviewed based on the following methodologies: classical optical transmission and reflection gratings, arrayed waveguide gratings, static Fabry-Pérot (FP) filter arrays, MEMS tunable FP interferometers, MEMS tunable photonic crystals, plasmonic filters, and fiber tip sensors. The comparison between the selected concepts concentrates on (i) the minimum space required for a particular spectral range, (ii) resolution, (iii) the integration in optical fiber technology, (iv) tunability to save space, (v) efficiency in using available light, (vi) multiplexing, (vii) miniaturization limits, and (viii) the potential of nanoimprint for cost reduction. Technologies for enhancing efficiency to obtain more available light and their applicability to the different methodologies were studied.
\end{abstract}

Keywords: miniaturized optical sensors; Fabry-Pérot interferometers; tuning range; MEMS; efficiency; nanoimprint; integration in fiber optics; multiplex advantage; photonic crystals; plasmonics

\section{Introduction}

Wearable interferometric sensors require a small size, high selectivity, and sensitivity if biomedical applications are envisaged. These three requirements often involve counterrunning demands and have previously been found to be difficult to satisfy in a single system. Such a limitation is also relevant in many other application fields of photonic methodologies: highly accurate metrology; health and environmental monitoring; food sensing; quality control in industrial fabrication; smart personal environments; and high data rate communication technologies. Wearable interferometric sensors are characteristically lightweight with a small footprint as a result of miniaturization, which at the same time enables low-cost, precision, and high efficiency. Photonic sensorics [1-10] enable extraordinary sensitivity, outstanding selectivity, and broad application fields [1] such as industrial production control, environmental trace gas detection, agriculture growth monitoring, medical prevention and medical diagnosis, and fiber optic communication technologies. Many of the optical sensors use fiber or optical waveguide technology and are already integrated in communication systems, with high 
potential for future integration in wearable interferometric sensors or in devices used for smart personal environments. However, a key challenge remains that of combining their outstanding performance in terms of resolution and efficiency while being miniaturized as much as possible at the same time.

In this review, methodologies of optical sensing in the near-infrared (NIR), visible (VIS), and ultraviolet (UV) spectral wavelengths ranges are introduced. This review also refines one of our recent papers [11], extending it toward integration into optical fiber technology and the improvement of the estimation of minimum size requirements, as well as formulates new insight into tunability efficiency.

A systematic overview of the different spectroscopic sensing principles is shown as a block diagram in Figure 1. In principle, the wide variety of sensor types can be categorized into different schemes. From a physical point of view, classification in accordance with the optical working principles is appropriate. For potential economic exploitation, classification with respect to robustness, complexity, recording and analyzing speed, potential applicability for high volume manufacturing, and the consideration of price aspects would be rather helpful. The block diagram shown here distinguishes in the first level between different optical working principles and focuses in the second level on sensor concepts that are particularly suitable for miniaturization and compatible with waveguide technology. The sensor concepts listed in the bottom row are considered in detail in this contribution.

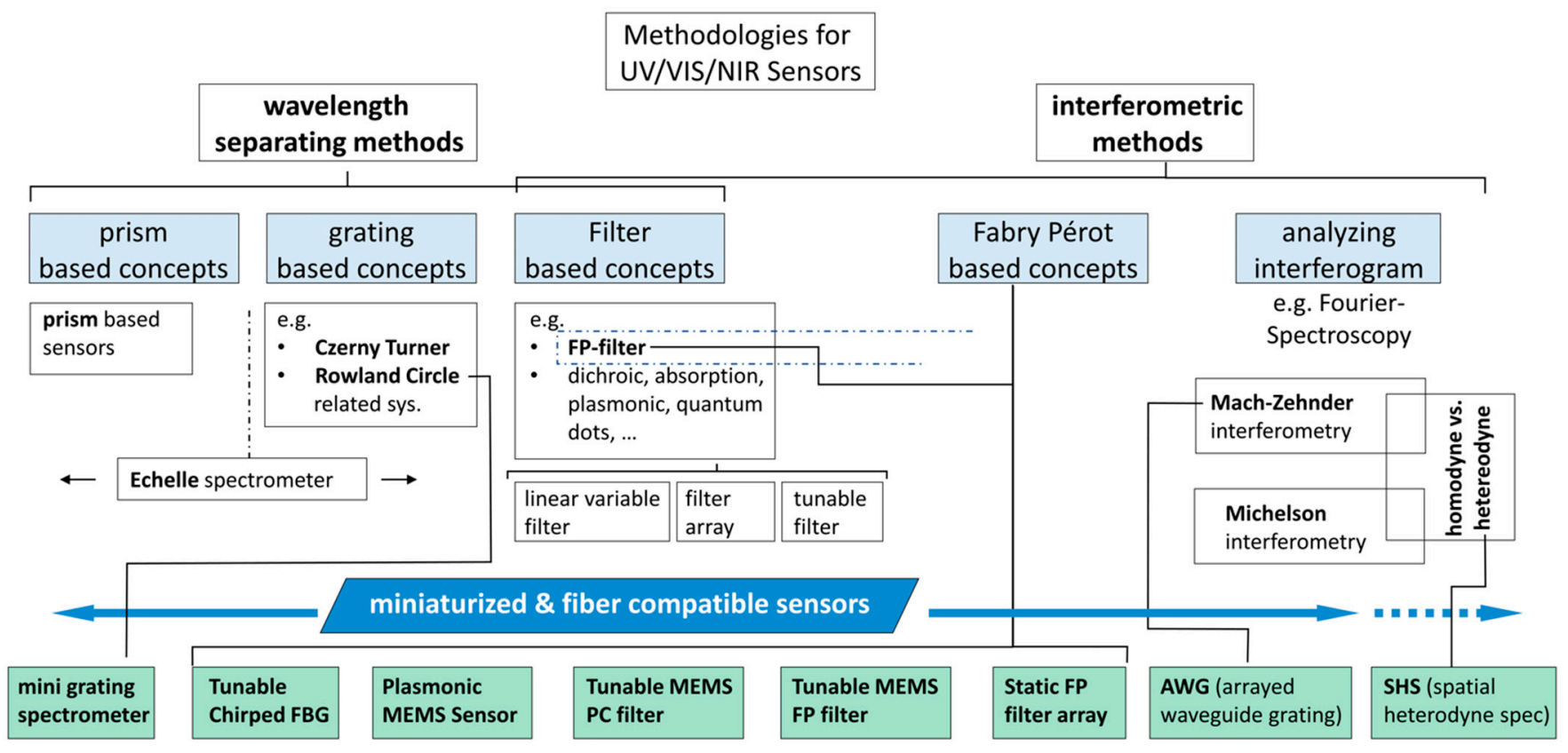

Figure 1. Block diagram of the sensor and spectrometer types considered in this review. This diagram should provide a general overview and an orientation throughout this extensive paper.

Traditional optical spectrometers [4,8-10] use prisms or gratings as dispersive or diffractive elements to dissolve the studied optical information into a spectrum (intensity versus wavelength plot). A transmission grating is depicted in Figure 2. As a general rule, a higher grating order means higher resolution; however, unfortunately, this also means a smaller intensity. To overcome this major disadvantage, the gratings are often blazed (exhibiting e.g., a saw-tooth structure) to transform the desired highest intensity from the zero order into higher diffraction orders [4]. This enables a rather high resolution and a relatively high intensity to be achieved at the same time. 


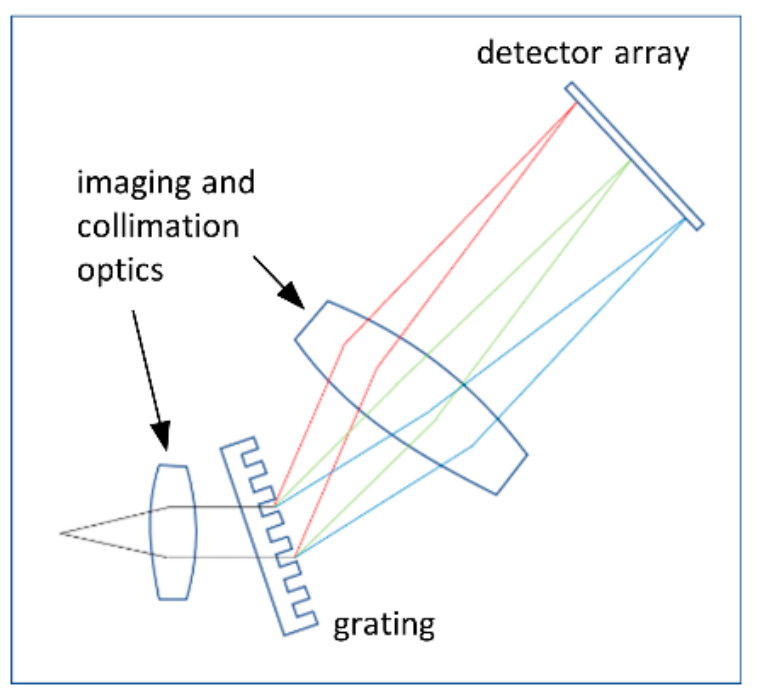

Figure 2. Schematic design of a transmission grating spectrometer with a symbolic grating shape.

There are different options to miniaturize the sensors, and Micro-Electro-Mechanical Systems (MEMS) represent one miniaturization option that enables wavelength tuning. In particular, FP and photonic crystal sensor systems or grating spectrometers can be miniaturized using MEMS. Actuation in MEMS-based devices was demonstrated for different material systems and wavelength ranges using different methodologies such as thermal, electromagnetic, piezoelectric or electrostatic actuation methodologies. Even in traditional grating spectrometers, MEMS can be applied to rotate the grating [12-15] and enable good performance.

More advanced methodologies use interferometry [3,4,16-20] for optical sensing based on the Mach-Zehnder, Fabry-Pérot, Michelson, and Sagnac principle. Additionally, there are many others which are closely related to these concepts. In the Michelson interferometer $[3,4]$ the incident light beam (input) is split into two parts that are reunified again at a later stage before reaching the photodiode sensor as the output beam. Precisely delaying one of the two beams generates a distinct phase shift, though a proper calibration has to be completed before using the interferometric sensor. Next, the interferogram (intensity as a function of the varying phase delay) is converted into the wavelength range, thus, delivering the spectrum of the input. The smaller the considered light wavelength, the higher mechanical precision required to obtain the phase shifts. Therefore, the involved mechanics is less complex in the NIR compared to the VIS range. Fourier spectroscopy [3] uses the Michelson interferometer in the NIR, in which the interferogram is the Fourier transform of the input signal [3].

The input beam is also separated into two branches in the Mach-Zehnder interferometer $[19,20]$. Likewise, the two waves propagating through the branches experience different optical path lengths (defined as the product of refractive index and physical length). The two beams are then reunified, and the varying phase shifts between the two beams result in different corresponding intensities of the output beam. Integrated Mach-Zehnder interferometers can be implemented using ridge or embedded waveguides. Today, integrated Mach-Zehnder interferometers are often applied as modulators or sensors and were demonstrated in many various materials such as $\mathrm{Si}, \mathrm{InP}, \mathrm{LiNbO}_{3}$, dielectric materials, inorganic glass, organic glass and polymers. By integrating a plate capacitor to one of the waveguide branches, the effective refractive index of that waveguide branch can be tuned via the capacitor voltage (i.e., the variable electrical field), making use of the electrooptic effect. Tuning the voltage produces an interferogram, and Fourier transforming the interferogram gives the spectra. A very important variant of the Mach-Zehnder interferometer is the derived arrayed waveguide grating (AWG) [21-27]. The AWG and Mach-Zehnder interferometers are perfectly adapted for use in fiber optical systems. There- 
fore, the AWG represents a significant waveguide-based sensor type and a basic device in dense wavelength division multiplex (DWDM) systems for present high-speed and high bitrate optical data and telecommunication systems. More than 100 waveguide branches of tailored lengths can be combined in an AWG. These interferometric devices are also named phased arrays. Yoshikuni et al. have contributed pioneering work in the field of AWGs for fiberoptic telecommunication in the $1.5 \mu \mathrm{m}$ communication bands, in which channel spacings of $50 \mathrm{GHz}$ were achieved [22-24]. AWG consist of $M$ waveguides (related to $M$ fiberoptic DWDM channels) of different optical path lengths which are introduced not by the effective refractive index, but by the varying physical lengths. The individually tailored lengths lead to distinct phase shifts at the output of the different waveguides. The waveguide couples the waves out into a free beam section in which (mode) confinement is only given by a vertical waveguide structure. At the opposite end of the free beam section, light constructively interferes only at specific positions. Another $M$ waveguides are beginning exactly at these positions, representing the output ports. The waveguides are spectrally ordered. Therefore, each individual output waveguide only guides light in an interval of $\Delta \lambda i$ with a central wavelength $\lambda i$ (vacuum wavelength), where $i=1 \ldots M$. These Mach-Zehnder and AWG sensors constitute a second interferometric sensor design.

The third sensor design, based on the Fabry-Pérot (FP) principle [4,28-58], uses also interferometric methodologies and is well suitable for fiberoptic sensors. Although no beam splitters are involved and only two transparent dielectric parallel mirrors are present, the FP interferometer involves multipath interference and is highly complex. The important optical element embedded between the two mirrors is the FP cavity. Inside the FP interferometer cavity, some wavelengths are dimensioned in a way that they can constitute standing light waves, and thus fulfilling the FP condition. For a vacuum (air) cavity and two perfectly flat metal mirrors embedding the cavity, the condition can be described by a simple formula: a multiple of half of the vacuum wavelength equals the cavity length. These explicit standing wavelengths are the cavity modes. However, in real conditions which have to be considered in interferometric sensorics, the modes (standing waves) penetrate into the dielectric mirrors. Even by replacing the vacuum wavelength in the FP condition with the wavelength in the medium (vacuum wavelength divided by the cavity refractive index), it is still a very rough approximation. This phenomenon is detailed later in Section 10. The wavelength of each specific mode (standing wavelength) passes the interferometer nearly unattenuated (almost $100 \%$ of the input intensity). It is quite common to refer to FP interferometers as FP filters, where the modes are also defined as FP filter lines. The reason for a specific wavelength passes the filter unattenuated and unreflected is due to constructive interference of all multipath waves at the FP output. All the remaining wavelengths which do not constitute modes experience destructive interference at the output. They are reflected by the FP filter and show constructive interference in the reflected beam. By shifting one of the mirrors precisely parallel along the optical axis, the corresponding spectrum, i.e., a spectral tuning of the wavelength, is obtained. Therefore, this interferometer also constitutes a spectrometer. By applying MEMS technology, wavelength tuning can be obtained by displacing one or both mirrors using MEMS actuation [37-57].

Adding optical substructures in the nanometer range onto membranes or cantilevers of MEMS offers further options to optimize the optical sensor assets. As the substructure size is roughly a factor of 100 below the typical dimensions of MEMS membranes or cantilevers, an integration of substructures is not affecting the general contour or functionality of these MEMS sensors. Often, 1D or 2D photonic crystal (PC) structures are integrated into membranes to enable specific guided mode resonances (GMR) $[59,60]$. In this case, the released MEMS layer is a slab waveguide with light waves incident in normal direction to the interfaces of the slab. Without integrating a PC structure, the free space mode is not able to couple to a guided mode in the slab. In contrast, it will be reflected and transmitted at each interface, constituting a typical thin-film spectrum. If a grating pattern with a period in the range of the desired wavelength range is added, this will lead to resonant coupling 
of a part of the incident wave into a leaky mode inside the slab. Because of its leaky nature, the mode will couple back-out of the slab-into both vertical directions and superimpose with the residual incident and transmitted free space mode (known as continuous mode). This results in a filter resonance line with Fano characteristics, both in transmission and reflection [61]. If the resonant coupling conditions are tailored appropriately, line shape and spectral width are influenced. Narrow linewidths are accomplished by applying low coupling strengths, while broadband reflections can be obtained using strong coupling and applying an overlap of several individual resonances. Sensors using the GMR can be strongly miniaturized [62] since they replace vertical periodic patterns of distributed Bragg reflectors (DBR) by horizontal pattern inside a single membrane. This means that they translate full FP filters into a narrowband Fano resonance of a PC slab. However, their fabrication and application are a real challenge due to their strong angle dependency. Therefore, use of very flat membranes or cantilevers is crucial, but this effect also restricts the acceptable angular spectrum of the incident light beam. As a consequence, the spot diameter and related divergence which can be used are restricted.

If the axial symmetry of $90^{\circ}$ is disturbed in the PC-based sensors or in the sensor lattice itself, altered conditions are obtained for in- and out-coupling along the $\mathrm{x}$ - and $\mathrm{y}$-direction, thus enabling further polarization selectivity of the sensor element. In the simplest case, this is obtained by a line grating (1D PC). A higher degree of control on coupling properties is achieved by 2D PC patterns with elements owning elliptical [63-65] or keyhole [66] shapes. Please note that the $\mathrm{x}$ - and $\mathrm{y}$-coordinates are spanning the in-plane directions. An alternative to enable polarization selectivity is using sub-wavelength structures. In this case, the interaction with the incident waves is defined by the effective refractive index method. Appropriate designs of the pattern disrupt the $90^{\circ}$ symmetry, leading to different effective indices for transverse magnetic (TM) and transverse electric (TE) polarization, and subsequently resulting in structural birefringence [67].

Nano-optic effects may be also used to substitute the dispersive elements of sensors. Surface-plasmon-polaritons (SPP) reveal resonances and interact efficiently in the optical nearfield. However, they experience the same critical trade-off between resolution of grating spectrometers and their ability for downscaling because they are based on the angular dispersion of the spectrum as well. In addition, the standard Kretschmann method of excitation makes downscaling quite difficult due to the required bulky prisms. The problem was addressed using a metallic grating coupler for surface plasmons positioned on a scanning MEMS cantilever, and then to read out the influence on the photodiode current of an integrated photosensor [68]. An alternative to improve the resolution of miniaturized spectrometers is achieved via the super prism effect in PC structures. The dispersion properties of a periodic $2 \mathrm{D}$ pattern can be designed to be much more pronounced than those of a 1D grating if the shape of the photonic bands is appropriately tailored close to the bandgap [69]. Nonetheless, it was shown that with 1D structures in form of chirped, resonant, or general layer stacks, a strong super prism effect is possible as well [70].

Continuing with this general overview of optical sensing, methodologies and instrumentation, the focus is now placed on the miniaturization potential of grating spectrometers. Concerning size, optical grating spectrometers used in the NIR, VIS, and UV span a length between several $\mathrm{mm}$ and a few meters. The optical resolution $\Delta \lambda / \lambda$ of the grating spectrometer is obtained by multiplying the diffraction order $n$ with the number of illuminated grating periods $N$, approximately. If we have to miniaturize the grating spectrometer, we are forced to reduce $N$ since it is impossible to reduce the grating period (the application defines the wavelength range we have to consider). In addition to the resolving power of the grating, the pixel sizes and the path lengths determine the resolution of the spectrometers, as can be seen in Figure 2. Thus, we can only reduce the size of the grating, and this means to reduce the total number of grating lines $N$ and to shorten the optical path length inside the spectrometer. Decreasing $N$ reveals a strong and negative impact on the spectral resolution. The first two photos (from left) in Figure 3 display the mini transmission grating spectrometer PEBBLE from the company Ibsen Photonics, Denmark [71] and the mini 
reflection grating spectrometer C10988MA-01 from the japanese company Hamamatsu Photonics, in which that of Hamamatsu reveals stronger miniaturization. In the second column (from left) in Figure 3, data from the two smallest grating spectrometers, C12880MA and C14384MA [72], are included. Currently, the C14384MA-01 and the PEBBLE are most probably the smallest grating-based optical mini spectrometers available in the market. The corresponding package dimensions of the C14384MA-01 are located in the sub-centimeter range. Scaling down this spectrometer could only be accomplished at the expense of the grating size, i.e., to work with a rather limited number of grating lines $N$. For the spectral range of $540-1050 \mathrm{~nm}$, the datasheets of the spectrometer reveal full width at half maximum (FWHM) of 17-25 nm (equivalent to linewidths) and corresponding resolutions $\Delta \lambda / \lambda$ of $42-56$. This sensor type makes the most efficient use of available light compared to all other sensor methodologies, and it is compatible with fiberoptic systems since it is also available with fiber pigtails. However, if strong miniaturization is required, the achievable resolution is often not sufficient: As already mentioned, grating spectrometer resolution and is strongly decreasing with shrinking size. Luckily, there are many alternative methodologies providing high resolution independent of size, and some of them will be discussed and compared in this review.

Concerning wavelength dispersing options for fiberoptic, sensor principles which (i) are compatible to fiber technology, (ii) have high resolution, and (iii) reveal strong miniaturization potential are considered. The following alternatives are included (and summarized in Figure 3): Static FP filter arrays on complementary metal oxide semiconductor (CMOS) sensor arrays or charge coupled devices (CCD) [28-37], MEMS tunable FP filter arrays [38-57] on photodetector (PD) arrays, AWG structures [21-27], MEMS tunable photonic crystal (PC) filters [62,73-77], plasmonic MEMS cantilevers [68] and thermally tuned chirped fiber Bragg gratings (FBG). Compared to the mini grating spectrometers mentioned above, all these alternatives reveal higher resolution, lower FWHM, and are much smaller in size. The content of Figures 1 and 3 will be considered throughout the whole review. Please note that there are other alternatives than those mentioned in Figure 3, such as MEMS grating spectrometers or Fourier spectrometers which were also strongly miniaturized in the past. However, these are beyond the scope of our paper. The focal points of our review are (i) to describe their method of integration into optical fiber systems, (ii) to show alternative fabrication technologies such as nanoimprint, (iii) to enlarge wavelength tuning behavior, (iv) to identify limits for optical sensor miniaturization, and (v) to envisage performance improvement and cost reduction.

This review emphasizes FWHM, resolution, and potential miniaturization limits with the focus on FP-type interferometers, and delivers various quantitative comparisons. The efficiency in using available light is discussed in detail and the different sensor methodologies are compared with respect to efficiency in this review. There is another recent and well elaborated review paper about miniaturized spectrometers [78] emphasizing the classification of spectrometer methodology and focusing on computational (reconstructive) and Fourier transform methodologies. It is more qualitative rather than quantitative with regard to miniaturization limits, since it only gives the extension in 1D. In contrast, our review gives the required area in 2D. Efficiency is not included in the scope of [78], but it is crucial for signal-to-noise ratio. Thus, our review and the review [78] ideally complement each other. 


\begin{tabular}{|c|c|c|c|c|c|c|c|c|c|}
\hline \multirow{2}{*}{\multicolumn{2}{|c|}{\begin{tabular}{|} 
Miniaturized spectrometers \\
for larger spectral ranges
\end{tabular}}} & $\begin{array}{c}\text { Mini } \\
\text { Spectrometer }\end{array}$ & $\begin{array}{c}\text { Mini } \\
\text { Spectrometer }\end{array}$ & AWG & $\begin{array}{c}\text { Static FP filter } \\
\text { array }\end{array}$ & $\begin{array}{l}\text { Tunable MEMS } \\
\text { FP filter }\end{array}$ & $\begin{array}{l}\text { MEMS tunable } \\
\text { PC filter }\end{array}$ & $\begin{array}{c}\text { Plasmonic MEMS } \\
\text { Sensor }\end{array}$ & $\begin{array}{c}\text { Tunable } \\
\text { Chirped FBG }\end{array}$ \\
\hline & & & & & & & & & \\
\hline \multicolumn{2}{|c|}{ Operation principles } & Transmission Grating & Reflection Grating & Mach Zehnder interf. & FP interferometer & $\mathrm{FP}$ interferometer & Photonic crystal PC & Plasmonic coupling & Grating phase shift \\
\hline \multicolumn{2}{|c|}{ Spectral range } & $\begin{array}{l}340-830 \mathrm{~nm} \text { [71] } \\
950-1700 \mathrm{~nm}[71]\end{array}$ & $\begin{array}{c}340-850 \mathrm{~nm} \text { [72] } \\
640-1050 \mathrm{~nm}[72]\end{array}$ & $\begin{array}{c}643-863 \mathrm{~nm}[26] \\
1530-1565 \mathrm{~nm}\end{array}$ & $400-1700 \mathrm{~nm}$ & $200-2000 \mathrm{~nm}$ & $1290-1340 \mathrm{~nm}$ & $550-1700 \mathrm{~nm}$ & $200-2000 \mathrm{~nm}$ \\
\hline \multicolumn{2}{|c|}{ Active tuning range } & - & - & - & - & $221 \mathrm{~nm} @ 1.5 \mu \mathrm{m}[56]$ & $30 \mathrm{~nm}$ & 300 nm @ $1.4 \mu \mathrm{m}$ [68] & $16.5 \mathrm{~nm} @ 1.5 \mu \mathrm{m}[79]$ \\
\hline \multirow{2}{*}{$\begin{array}{c}\text { FWHM } \Delta \Lambda \\
\text { typical values }\end{array}$} & VIS & $6 \mathrm{~nm}[71]$ & $13 \mathrm{~nm}[72]$ & $0.1 \mathrm{~nm}$ & $2.5 \mathrm{~nm} @ 500 \mathrm{~nm}$ & $2 \mathrm{~nm} @ 600 \mathrm{~nm}$ & - & - & - \\
\hline & NIR & $12 \mathrm{~nm}[71]$ & $20 \mathrm{~nm}[72]$ & $0.4-0.8 \mathrm{~nm}[24]$ & 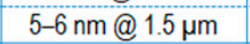 & $3 \mathrm{~nm} @ 1.5 \mu \mathrm{m}$ & - & - & - \\
\hline \multirow{2}{*}{$\begin{array}{l}\mathrm{FWHM} \Delta \Lambda \\
\text { record values }\end{array}$} & VIS & $\begin{array}{l}4.3 \mathrm{~nm} @ 480 \mathrm{~nm}[71] \\
4.2 \mathrm{~nm} @ 725 \mathrm{~nm}[71]\end{array}$ & $\begin{array}{c}12 \mathrm{~nm} @ 420 \mathrm{~nm} \\
{[72]}\end{array}$ & $0.05 \mathrm{~nm}[26]$ & 1 nm @ 500 nm & $1 \mathrm{~nm}$ single air-gap & - & - & - \\
\hline & NIR & 看 & $\begin{array}{c}17 \mathrm{~nm} @ 950 \mathrm{~nm} \\
{[72]}\end{array}$ & $\begin{array}{c}0.22 \mathrm{~nm} @ 1.5 \mu \mathrm{m} \\
{[22]}\end{array}$ & $5 \mathrm{~nm} @ 1.5 \mu \mathrm{m}[36]$ & $\begin{array}{l}0.1 \mathrm{~nm} \operatorname{lnP} / \text { mult air- } \\
\text { gaps @ } 1.5 \mu \mathrm{m} \text { [48] }\end{array}$ & $\begin{array}{c}0.08 \mathrm{~nm} @ 1.3 \mu \mathrm{m} \\
{[77]}\end{array}$ & $10 \mathrm{~nm} @ 1.3 \mu \mathrm{m}[68]$ & $\begin{array}{c}0.007 \mathrm{~nm} @ 1.5 \mu \mathrm{m} \\
{[79]}\end{array}$ \\
\hline Resolution VIS & $N \Delta \lambda$ & $100-170[71]$ & $35-50[72]$ & - & 500 & - & - & - & - \\
\hline Resolution NIR & $N \Delta \lambda$ & $\sim 100[71]$ & $42-56[72]$ & $7000 @ 1.5 \mu \mathrm{m}$ & $300[36]$ & $300-15000$ & $3000-10000$ & $100-150$ & 220000 \\
\hline \multicolumn{2}{|c|}{ Production challenge } & $\begin{array}{l}\text { Automation. } \\
\text { The rest are } \\
\text { proprietary } \\
\text { information }\end{array}$ & $\begin{array}{c}\text { proprietary } \\
\text { information; most } \\
\text { probably alignment } \\
\text { of PD array and } \\
\text { blazed grating }\end{array}$ & $\begin{array}{l}\text { to achieve effective } \\
\text { refractive index } \\
\text { homogeneities in the } \\
\text { individual ridge } \\
\text { waveguides }\end{array}$ & $\begin{array}{l}\text { perfect resist filling of } \\
\text { templates, residual } \\
\text { layer homogeneity or } \\
\text { effort in individual } \\
\text { etching and deposition }\end{array}$ & $\begin{array}{c}\text { flat membranes, } \\
\text { vertical stress gradient } \\
\text { compensation }\end{array}$ & $\begin{array}{l}\text { QD spatial positioning } \\
\text { and adjusting its } \\
\text { spectral line, e-beam } \\
\text { lithography serial } \\
\text { writing }\end{array}$ & $\begin{array}{c}\text { flat cantilever, stress in } \\
\text { metal layer, large angle } \\
\text { stroke }\end{array}$ & $\begin{array}{c}\text { fabrication of precise } \\
\text { chirp in fiber, positioning } \\
\text { and actuation of heater } \\
\text { element }\end{array}$ \\
\hline \multicolumn{2}{|c|}{ Nanoimprint applicable } & probably & no & yes & yes & yes & yes & yes & no \\
\hline \multirow{2}{*}{$\begin{array}{l}\text { Potential space } \\
\text { requirements to cover } 5\end{array}$} & $400 \mathrm{~nm}$ in vis & $43 \mathrm{~mm}^{2}$ & $200 \mathrm{~mm}^{2}$ & $90 \mathrm{~mm}^{2}$ & small $0.07 \mathrm{~mm}^{2}$ & small $0.06 \mathrm{~mm}^{2}$ & - & - & - \\
\hline & $500 \mathrm{~nm}$ in NIR & $43 \mathrm{~mm}^{2}$ & $46 \mathrm{~mm}^{2}$ & $50 \mathrm{~mm}^{2}$ & small $0.09-1 \mathrm{~mm}^{2}$ & small $0.05 \mathrm{~mm}^{2}$ & small $0.33 \mathrm{~mm}^{2}$ & medium $9.5 \mathrm{~mm}^{2}$ & large $300 \mathrm{~mm}^{2}$ \\
\hline \multirow{2}{*}{ package size } & in vis range & medium & large & large & small & small & - & - & - \\
\hline & in NIR range & medium & medium & large & small & small & small & medium & very large \\
\hline \multicolumn{2}{|c|}{ Current and tendative price } & $600 €$ PEBBLE vis & $500 € \quad$ C12880MA & high & low & high & high & medium & medium \\
\hline \multicolumn{2}{|c|}{ Yield } & $100 \%[80]$ & high & medium & high & medium & medium & medium & high \\
\hline \multicolumn{2}{|c|}{ Efficiency in available light } & $\begin{array}{c}\text { very high } 70-80 \% \\
{[80]}\end{array}$ & very high & medium & weak, can be boosted & weak, can be boosted & weak, can be boosted & weak, can be boosted & medium \\
\hline
\end{tabular}

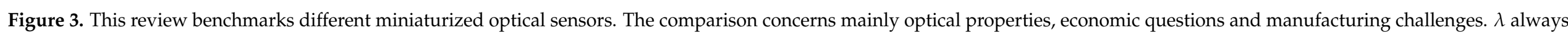

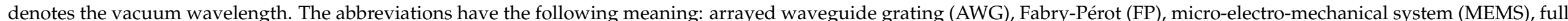

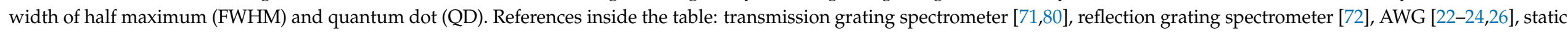
FP filter [36], tunable MEMS FP filters [48,56], MEMS tunable PC filter [76], plasmonic MEMS sensor [68], tunable chirped FBG [79]. 
The review is organized as follows: Section 2 deals with FP filter methodologies, microspectrometers based on FP filter arrays, and our nanospectrometer based on static FP filter arrays which is fabricated by nanoimprint. The digital fabrication methodology for cavity arrays of different heights is demonstrated for the technological fabrication of 3D nanoimprint templates by digital etching in Section 3. This methodology is applied for the fabrication of static FP filter arrays in the VIS spectral range and is also demonstrated in Section 3. Here, a single nanoimprint is demonstrated over three DBR stacks of different design wavelengths and thus different total heights. Section 4 includes experimental results of static FP filter arrays in the VIS range. In Section 5, the design an FP filter array on a detector array with mounted fiber is shown. Section 6 deals with the question to make the most of available light. A methodology is demonstrated which partially compensates for the low efficiency in using available light. This weakness is characteristic for many high resolution optical sensors which have the ability of being strongly miniaturized without loss in resolution. Efficiency boosting is demonstrated by spectral preselection and shown in a laboratory demonstrator. Section 7 briefly describes differences in fabrication of static FP filter arrays if we switch from the VIS to the NIR spectral range. Section 8 describes single air-gap MEMS tunable FP filters for the VIS and NIR spectral range. Section 9 presents methodology, fabrication and characterization of InP multiple airgap MEMS tunable sensors for the NIR. Section 10 provides a broader view on the limits of MEMS tunable FP filters regarding materials and geometric design. Section 11 contains an overview of further concepts for miniaturized optical sensors based on plasmonics, ring resonators, quantum dots, spatial heterodyning, and photonic crystals on fiber tips and in MEMS membranes. Section 12 estimates and illustrates potential space requirements for different sensor methodologies with maximum miniaturization. For the optical sensors shown in Figure 3, the potential space in the area is calculated according to the requirement to cover $400 \mathrm{~nm}$ in the VIS spectral range and $500 \mathrm{~nm}$ in the NIR for the case of maximum miniaturization. Section 13 deals with the limits of 2D and 3D nanoimprint lithography and discusses the strength of nanoimprint to reduce fabrication time and cost with regard to the six sensor types compared in Figure 3. Subsequently, the limits of wide wavelength tuning are summarized.

\section{Methodology of Static FP Sensor Arrays}

The static FP sensor array consists of an FP filter array on a photodiode CMOS array or a CCD array. In the literature, these static FP sensor arrays are also called microspectrometers or nanospectrometers, depending on whether or not nanoimprint technology is applied in the fabrication.

\subsection{Microspectrometers}

An FP filter interferometer is defined as a transparent resonance cavity layer embedded in between two highly reflective mirrors. Within the high reflective spectral range (stopband) defined by the DBRs, only one or several filter lines are allowed to transmit with almost $100 \%$ transmission. Each filter transmission line is characterized by its FWHM and its spectral transmission peak (maximum of the filter line). The position within the spectral stopband can be adjusted by a distinct choice of the cavity layer thickness. Please note that the term cavity height or cavity length are used as well in the literature. The longer the cavity, the longer the wavelength of the filter line. For the highly reflective DBRs, dielectric materials of low absorption loss are chosen. For each DBR, thin films are stacked with low and high optical refractive indices alternating in sequence. The higher the width of the stopband, the larger the contrast of refractive indices of the two chosen materials.

By combining an FP filter array and a detector array matching in size, a microspectrometer sensor is obtained. Miniaturizing these sensors does not reduce the spectral resolution as opposed to conventional grating-based spectrometers. These microspectrometers based on FP filter array [28-31] may require complicated fabrication steps for defining all the different heights of the filter cavities. Various methodologies were reported to generate the 
unequal cavity heights. Digital etching was used by Correia et al. [28,29] to fabricate 16 different FP cavity heights using four lithography and four corresponding etching steps (4 steps for 16 pixels). Digital deposition was applied by Wang et al. in 2007 [31] to define 128 different FP cavity heights with nine lithography and nine corresponding deposition steps ( 9 steps for 128 pixels). Digital masking [11] allows (i) digital etching if it is combined with a sequence of etching steps $[28,29]$ and (ii) digital deposition if it is combined with a sequence of deposition steps [31]. The higher the number of different cavity heights (being fabricated) is, the more filter transmission lines (pixels) can be included in an FP filter array. However, these high number of steps might not be cost-efficient in industrial fabrication.

\subsection{Nanospectrometers}

To significantly reduce the number of required fabrication steps by consolidating them into a single step, nanoimprint is used to define all required different cavity heights for a complete FP filter array [11,32-34]. Thus, nanoimprinted cavities are used with precisely defined heights between the two identical highly reflecting DBRs. From the usage of nanoimprint technology in their fabrication, we were introducing the term nanospectrometers-a combination of an FP filter array and its corresponding detector array-as shown in Figure 4a with a selection of four FP filters out of the whole array. The filter array comprises of two DBRs (shown in gray) and the cavities (shown in orange). The cavities were fabricated in a single nanoimprint step. Each individual filter cavity height defines the wavelength of a characteristic different narrow filter line. One option is to directly deposit the filter array on a detector array (black and magenta). In Figure 4a, each FP filter has one detector, constituting one pixel. However, each filter can also correspond to many detector pixels (e.g., 4, 9, 16, etc.). In Figure 4a, the right part displays the spectral transmission spectra of these four FP filters. Since they have the same DBR, they reveal the same spectral stopband widths (i.e., same spectral width of minimum transmission). However, the spectral positions of the filter lines vary due to the tailored different cavity heights. Within a single step, 3D nanoimprint allows fabrication of all different cavities (even 100,000 or more, theoretically). In the following, the static FP filter arrays are discussed in Sections 2-7 (fixed, i.e., static cavity heights), and the variable MEMS tunable FP filter arrays are considered in Sections 8 and 9.

a)

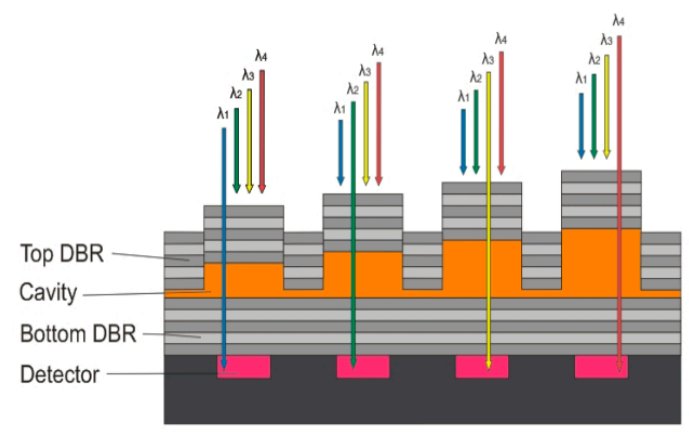

b)

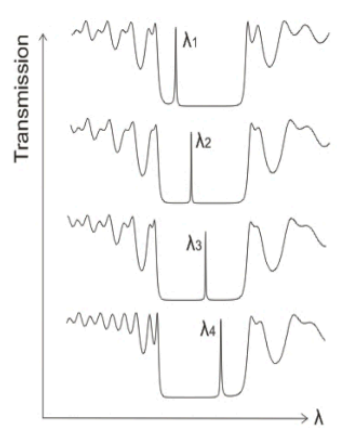

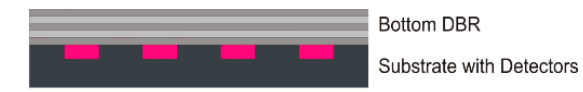

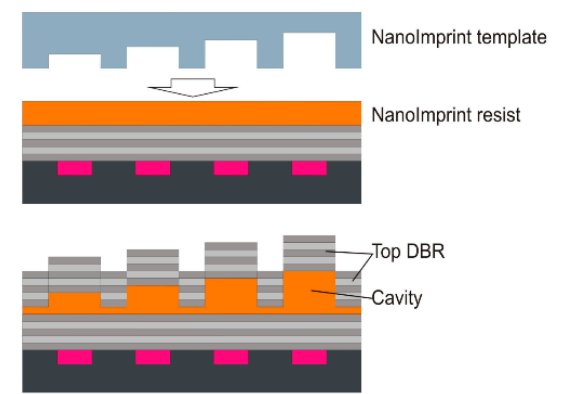

Figure 4. (a) Cross section Fabry-Pérot (FP) filter array with a detector array below constituting a nanospectrometer (left) and the related calculated transmission spectra (right); (b) The fabrication process uses Substrate Conformal Imprint Lithography (SCIL). Reprinted with permission from ref. [11]. Copyright 2021 MDPI.

Applying a single 3D nanoimprint step, 192 unequal cavity heights are defined with a single nanoimprint step, generating 192 pixels. This 3D nanoimprint does not require time-consuming digital etching [28,29] or digital deposition [31]. Since the nanoimprint template can be reused many times, this technology is cost-efficient. The digital etching is only used once to fabricate the 3D nanoimprint template, and not permanently during cavity fabrication. 
Figure $4 \mathrm{~b}$ illustrates the sequence of main fabrication steps from top to bottom. It starts with the deposition of the bottom DBR on the detector array. Next, the liquid cavity material (orange) is deposited by spin-coating. Subsequently, the 3D cavity structure is defined using a transparent 3D stamp (light blue) which is pressed into the cavity material and hardened via UV light. After lifting the stamp, the top DBR is deposited.

Nanoimprint is a process of shaping deformable materials by means of molding technology. Presently, many variants of nanoimprint technologies [81-84] are used to generate high resolution 2D structures. On the contrary, 3D nanoimprint is less common but essential for our nanospectrometer. It is challenging to accurately control the vertical dimension (3D) in addition to the lateral dimension. For mass production, a master template (as a positive), including in our case the checkerboard-type arranged mesa structures (various cavity structure heights), is replicated into many identical stamps (as a negative). The 3D nanoimprint is performed using one of these replicated stamps, generating the orange 3D cavity structures (again positive) simultaneously in a single step (Figure 4). Please note that the process described in Figure 4 is involving only a single DBR, thus, revealing a single stopband.

In the following section, three bottom DBR stacks which reveal different heights are imprinted in a single step to produce 192 different FP filters as a proof-of-principle. This imprints over the vertical steps located at the lateral borders of the DBR stacks, each of which has a different vertical extension.

\section{Static FP Filter Array Fabrication in the VIS Spectral Range Demonstrating a Single Nanoimprint over 3 DBR Stacks of Different Height}

\subsection{DBR Mirrors: Materials and Geometrical Issues}

Before fabrication, the filter array design has to be performed. The individual cavity heights were identified via simulations using "OpenFilters" [85] in the range of 26-215 nm. However, nanoimprint process is often associated with residual layers that has to be considered in the design. To keep the residual layer constant in lateral directions despite the different mesa volumes, four adjoining mesa heights are laterally grouped in a $2 \times 2$ submatrix consistently, where all sub-matrices of four mesas have the same combined volume. Thus, the lateral positioning of these cavities is done applying the volumeequalized design methodology [86] to ensure that the residual layer thickness is as constant as possible.

Since DBRs are used as highly reflecting mirrors, the cavity heights have to be designed in such a way that the transmission lines are within the spectral stopbands of the DBRs. If a DBR with a $100 \mathrm{~nm}$ stopband width is used, the different filter transmission lines must be spectrally located inside the stopband width of $100 \mathrm{~nm}$. In that case, the detectable range is a bit smaller than $100 \mathrm{~nm}$. As already mentioned, the DBR stopband width depends on the refractive index contrast of the used materials, which can be deposited for example via Plasma Enhanced Chemical Vapor Deposition (PECVD) or ion beam sputter deposition (IBSD) [34]. In the VIS spectral range, $\mathrm{SiO}_{2} / \mathrm{Si}_{3} \mathrm{~N}_{4}$ DBRs allows about $100 \mathrm{~nm}$ stopband width, and a $\mathrm{TiO}_{2} / \mathrm{SiO}_{2} \mathrm{DBR}$ enlarges the spectral stopband to about $200 \mathrm{~nm}$. It is important to mention that the interface quality of cavity and DBR mirror as well as the steadiness of the periods within a DBR are important for high optical quality of the sensor, by ensuring high filter line transmissions and low linewidths.

\subsection{Definition of 3D Nanoimprint Templates Using Digital Etching and Digital Deposition}

The fabrication of the 3D templates (corresponding to the design) is the most important part of 3D nanoimprint lithography. Since many different mesa height levels are required with height differences located in $\mathrm{nm}$ range and accuracies in sub-nm range, the fabrication of 3D templates is a challenge of enabling multiple mesa heights and accurate height differences in the nm range combined with accuracies in the sub-nm range. In comparison, it is by far less challenging to define $2 \mathrm{D}$ templates. A single step in e-beam lithography and subsequent etching are enough to fabricate $2 \mathrm{D}$ structures with a complex lateral pattern since a constant height can be obtained throughout the imprint area. However, fabricating 
3D templates is by far more challenging since the key requirements for the sensors are determined by the third dimension (height). The number of lithography and etching steps can be dramatically reduced if the heights are arranged in a digital way, allowing application of digital masking [34] during lithography, followed by a tailored etching step, respectively. More details can be found in [11], in which the fabrication of the master template (made of $\mathrm{Si}$ or GaAs in our case) is described.

The accuracy demand for the FP filter arrays is very low in lateral resolution and contrarily very high in vertical direction. A highly accurate etch depth control is required in the use of digital etching and lithography methodology for the 3D nanoimprint templates.

The digital lithography and etching methodology is used to define all the different checkerboard-like arranged mesa of different heights in the master template. As already mentioned, the master template is replicated to create stamps, thus transforming the different mesa into the required different cavity dimensions. Nanoimprinting with the stamps translates the stamp with its checkerboard-like cavities of different depths again into the different checkerboard-like arranged mesa morphology of different mesa heights.

As a comparison, Correia et al. [28,29] applied digital etching to directly fabricate the checkerboard mesa structure and Wang et al. [31] applied digital directly to deposit the checkerboard mesa structure. Such approach works as an initial demonstration of the principle; however, this would be not desirable for industrial production. A more efficient approach is using digital lithography and etching only to define the nanoimprint master template, which can be reused (replicated) multiple times; therefore it is well applicable for future mass production.

\subsection{Combining Three DBR Stopbands in the Fabrication Process of an FP Filter Array}

Often, the width of a single DBR stopband is not broad enough. In this case, combining several stopbands to extend to the usable total spectral range of the nanospectrometer is required. A proof-of-principle is demonstrated for this purpose, in which three spectrally neighboring stopbands are combined. 3D nanoimprint was performed across these three DBR stacks of different heights, and filter arrays were fabricated. In Figure 5, only three different FP filters out of many are depicted for each of three DBRs (with different stopbands). This results in nine individual cavities, displayed here as a part of a whole sensor device. Since the lower DBRs can be directly defined on a detector chip, expensive micro-mounting at later fabrication stage can be avoided.

For a DBR, the central wavelengths of the DBR stopbands are defined by the thicknesses of the thin quarter-wave films. A proof-of-concept with $\mathrm{SiO}_{2} / \mathrm{Si}_{3} \mathrm{~N}_{4} \mathrm{DBRs}$ fabricated by PECVD is shown, in which less than $120 \mathrm{~min}$ is required to deposit a single 9.5-period DBR in this material system. Structuring of the DBRs is performed by means of lithography, lift-off, and an etching process as described in detail in [11]. If the application requires more stopbands to enhance the total spectral range, this is also possible with additional lithography steps. The important point in fabricating sensors with multiple DBRs is simply to maintain a high fabrication quality of the devices even after repeating the process cycle several times.

According to Figure 5, the three bottom-DBRs with spectrally neighbored stopbands provide different vertical total heights of $224 \mathrm{~nm}$ and $188 \mathrm{~nm}$ for the step height (height difference) between bottom DBR 1 and bottom DBR 2 and the step height between bottom DBR 2 and bottom DBR 3, respectively. Using Substrate Conformal Imprint Lithography (SCIL) with flexible stamps, we performed 3D nanoimprint across these vertical steps. The flexible stamp combines hard-PDMS (polydimethylsiloxane) to ensure structure conformity and soft-PDMS connecting it to a flexible transparent carrier (i.e., thin glass or polymer), thus providing the large scale conformal property. In general, nanoimprint lithography enables large area imprinting up to 12 inch, and SCIL reaches 8 inch with resolutions below $10 \mathrm{~nm}$ [84]. The SCIL stamp can be reused for 500 prints using hard-PDMS and 600 prints using X-PDMS [84]. The tricky masking before depositing the next DBR is described in $[11,37]$ in detail. 


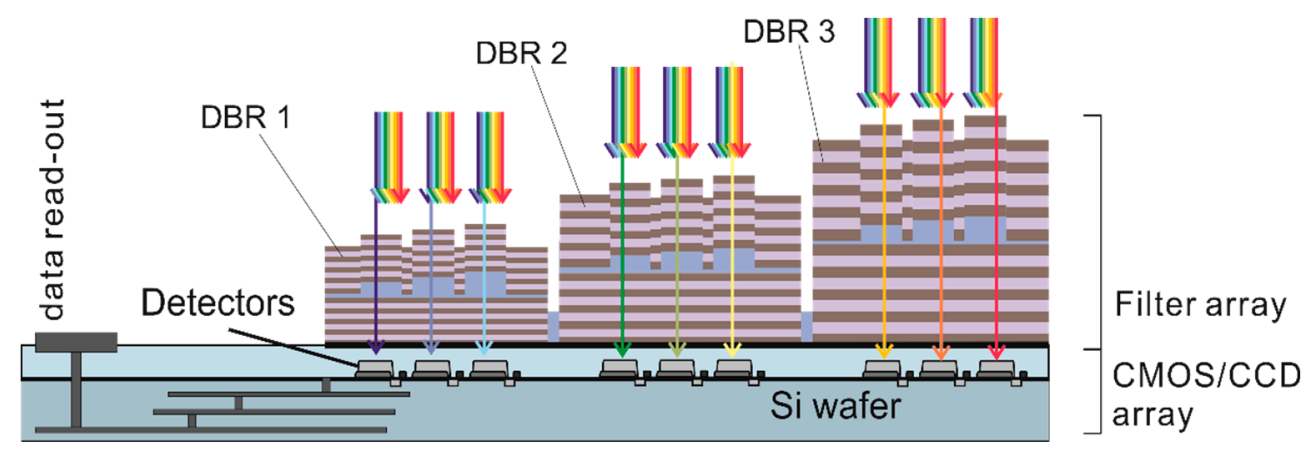

Figure 5. Cross section a nanospectrometer combining three different, spectrally neighbored DBRs in an FP filter array with a sensor array in CMOS or CCD technology. The definition of the blue cavity layer is achieved in just one nanoimprint step. The inclusion of nanoimprint for this sensor provides the term nanospectrometer, which we introduced for this sensor device. Shown here is the first option, namely by placing the FP filter array directly on the photodetector array including the processing integrated electronics. Reprinted with permission from ref. [11]. Copyright 2021 MDPI.

\subsection{The Complete Array: Lateral Arrangement of the FP Filters}

All FP filters of each of the three arrays are organized in a formation of $12 \times 12$ $(144)$ checkerboard. The central $64(8 \times 8)$ filters comprise 64 different cavity heights, while the residual 80 filters surrounding the central part are used as control elements for the nanoimprint quality and the residual layer thickness, only for research purposes. More details on this subject is provided in [11]. To estimate the minimum required space in Section 11, the lateral size of each mesa is considerably minimized. Each filter array containing 64 different cavity heights reveals 64 distinct transmission lines per array. A total of 192 different transmission lines were obtained by combining three stopbands. Please note that each of the 192 cavities has different heights and they were imprinted altogether via a single 3D nanoimprint process step. This enormously simplifies the fabrication process.

\section{Experimental Results of Static FP Filter Arrays in the VIS Range \\ 4.1. Transmission Spectra of Static FP Filter Arrays}

Optical spectra of all FP filter lines were recorded using a microscope spectrometer setup which includes a confocal microscope (Imager D1m Zeiss), a lateral active aperture manipulation, a halogen lamp, a photodetector, a lateral active aperture manipulation and a commercial grating spectrometer (HR 2000 Ocean Optics) with a resolution of $0.5 \mathrm{~nm}$ [35]. Figure 6 displays spectra of 192 filter lines of the FP filter array consisting of three DBRs (spectrally neighbored). The 192 filter lines cover a total spectral range of $163 \mathrm{~nm}$ (i.e., $507-670 \mathrm{~nm}$ ) with spectral increments of about $1 \mathrm{~nm}$ or below and without any gaps. The transmission intensities of the different filter lines vary due to an interplay between material absorption, linewidth variation, reflectivity changes, and further effects. The material absorption is higher for shorter wavelengths than that for longer wavelength since the strong material absorption of glass in the UV region is approached. This explains the strongly reduced transmission intensities with decreasing wavelength for each stopband. In addition, stopbands with larger central wavelength have thicker DBR stacks and thus suffer more from absorption. In contrast, stopbands with smaller central wavelength suffer less from absorption. This explains the trends measured in Figure 6. For details see $[11,37]$ 

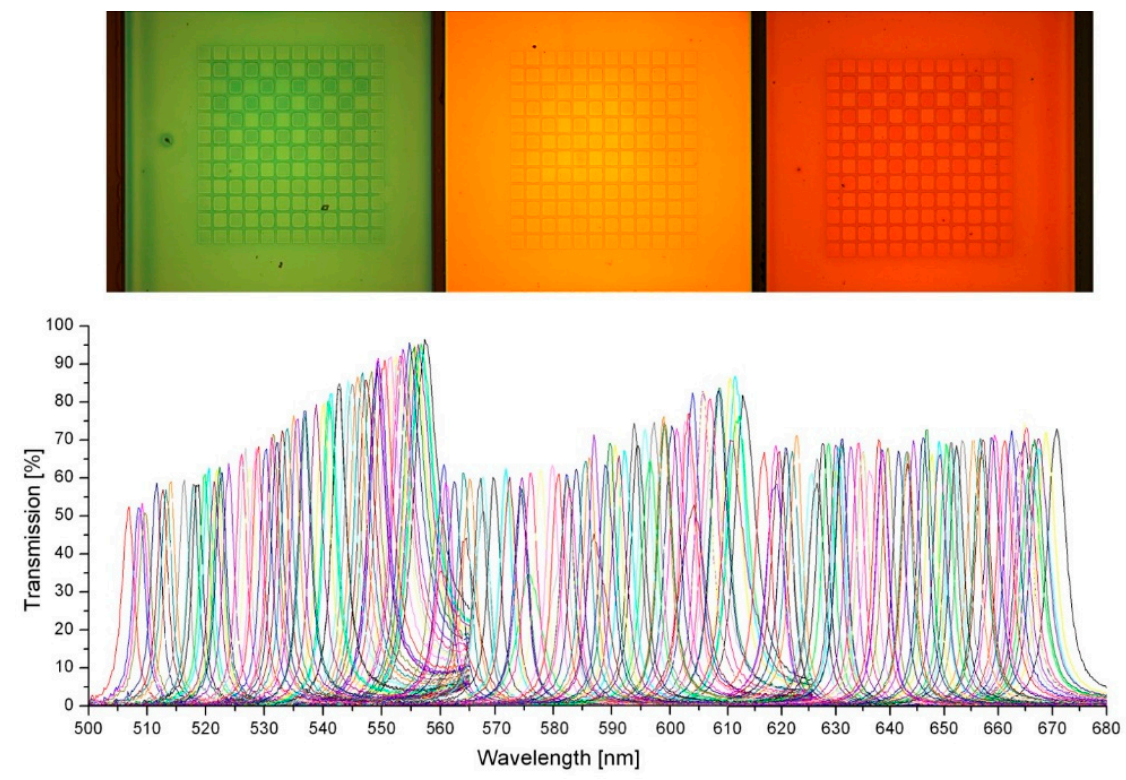

Figure 6. (Top) optical micrograph displaying three FP filter arrays and (bottom) related optical transmission spectra. Reprinted with permission from ref. [37]. Copyright 2018 Springer Applied Nanoscience.

\subsection{Interpretation of Experimental Results Concerning Linewidths}

The experimental linewidths (FWHM) presented in Figure 5 of [11] were measured in the FP filter array, as shown in Figure 6 in this review. The measured FWHM are found to be between 1.7 and $5 \mathrm{~nm}$. The closer we come to the borders of the stopbands, the higher the measured values. This observed strong variation of the FWHM as a function of wavelength is due to an interplay of many effects: reflectivity changes, spectral variation of material absorption interface roughness, and further effects.

The spectral reflectivity of each DBR is dependent on the precise spectral position inside its stopband, which is strongly influencing the dependence of FWHM on wavelength. The highest reflectivity exists at the center of the spectral stopband, leading to smallest FWHM at these spectral positions. As the filter lines come closer to the stopband borders, the lower the reflectivity, and thus the higher the FWHMs of the filter transmission lines. This is by far the most dominating effect and explains the main FWHM features that were observed experimentally.

Next, the role of interface roughness is discussed, first by considering one stopband in the VIS range. Assuming that the same interface roughness is occurring at the two interfaces between cavity and DBR for all the FP filters, the influence of interface roughness $\Delta l$ has a stronger influence on smaller cavity lengths $L$. For $L_{1}<L_{2}$, a larger relative cavity length fluctuation is obtained for smaller cavities $\Delta L_{1}=L_{1} \pm \Delta l$ than for longer cavities $\Delta L_{2}=L_{2} \pm \Delta l$. Increasing FWHM of a transmission line automatically leads to a reduction of the peak intensity. Since this effect is not clearly visible in the experiments, we conclude that the interface roughness is not very pronounced in our case. In addition, another effect is involved: the scattering probability increases with decreasing wavelength, since the averaging over interface roughness is more pronounced for larger wavelengths.

In our experiments, the lowest FWHM value of $1 \mathrm{~nm}[33,34]$ are observed; however, not in FP arrays as depicted in Figure 6, but in an FP filter processed individually. We estimate that smaller values down to $0.5 \mathrm{~nm}$ should be possible with 15.5 periods of $\mathrm{SiO}_{2} / \mathrm{TiO}_{2}$, using ultrapure $\mathrm{Si}$ and Ti targets and extended vacuum pumping.

\section{Static FP Sensor Array in a Fiber Technology System}

In our previous paper [11], a laboratory demonstrator is presented as a proof-ofprinciple. In the demonstrator, an FP filter array with optical bandpass filters was integrated into a commercial grayscale CCD camera and equipped with a telecentric lens. To 
implement the signal processing, a procedure similar to that described by Emadi et al. [87] is used. In [11], free beam optics was involved in remote sensing of fruit on a tree or bread inside an oven, whereas the focus in this review is on sensing integrated in fiber technology. Figure 7 presents the schematic design of a sensing system consisting of a nanospectrometer (FP filter array plus detector array), bandpass filters, and fiber input. The divergent light leaving the fiber is parallelized by an achromatic lens system. The bandpass filters block the spectral light outside the FP stopbands, which otherwise would also transmit, reach the Si detector array and increase the noise level. The electrical output and signal processing is not shown here. This was presented in our previous paper [11].

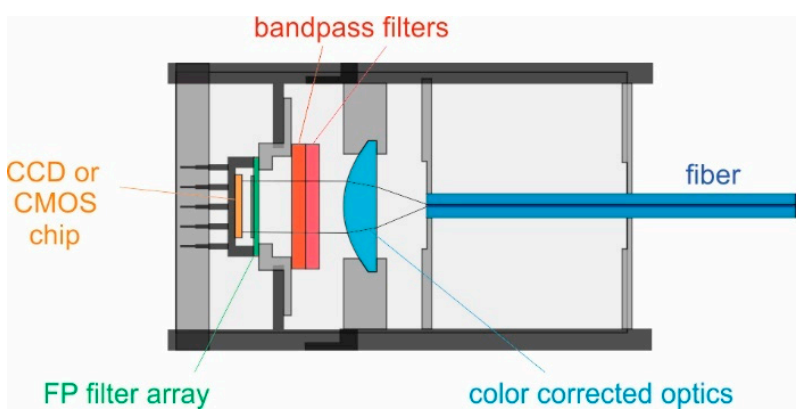

Figure 7. Schematic cross section of an FP filter array mounted parallel and in close vicinity of the Si detector array (CCD or CMOS). The divergent light of the fiber is made almost parallel by the lens system. In this option, the FP filter array is defined on a glass substrate which is aligned here in a bottom-up way on the CCD or CMOS photodiode array. As a comparison, in the first option shown previously in Figure 4 the FP filter array is defined directly on the detector array.

\section{Laboratory Demonstration of Efficiency Boosting by Spectral Preselection}

The benefits of the FP filter-based sensor concepts are their compactness with further miniaturization potential and their ability to detect a broad spectral range with high resolution. However, one considerable drawback of filter-based systems is their very low detection efficiency. Notwithstanding, this disadvantage is also typical for other interferometric or plasmonic sensor principles (Figures 1 and 3). This chapter presents a methodology for boosting the efficiency (making full use of available light), demonstrated for FP filter sensors. Moreover, it can also be applied to all methodologies suffering from the abovementioned disadvantage. In our previous paper [11], it was demonstrated in free beam optics methodology for remote optical sensing. In this paper, it is presented for fiber-based sensing systems.

The reason for this disadvantage is visualized in Figure 8a. The available "white" light to be analyzed is delivered by an optical fiber, where a parallel light bundle is subsequently produced by collimation optics. To record a complete spectrum, the light must be distributed over the entire area covered by the filters or filter arrays. Finally, the light arrives at the filter arrays or filter array groups (depicted as 1 to $N$ ). In this case, each narrowband filter receives the entire broadband spectrum, but only a small fraction of the incident light is transmitted through it and can be used for detection. This means that most of the analyzed light is reflected and lost for application. The narrower the transmission line of the filter, the greater the loss. As one solution to drastically improve the efficiency of filter-based systems, the spectral preselection concept was proposed [88]. Figure 8b demonstrates the basic principle of the preselection concept that comprises the spectral and spatial separation of the highly concentrated incoming light. In particular, the resulting partial spectra (subbands) are spatially separated and delivered to the corresponding filter arrays which only cover a limited spectral range. Each single filter acquires the increased intensity because the spectral preselection method concentrates the necessary wavelength region there, where it is most needed. As an example, Figure $8 \mathrm{c}$ shows the photo of the FP filter array [37] as one of the suitable filters for the described concept. 


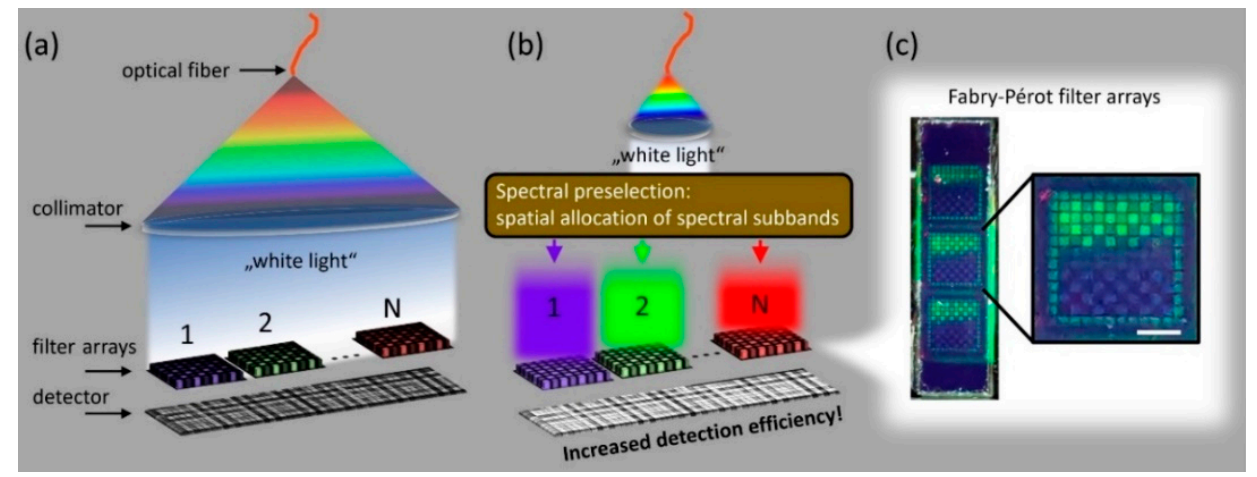

Figure 8. Basic principle of efficiency enhancement of filter-based spectrometers. (a) Typical case without spectral preselection: filter arrays are illuminated with broadband light. (b) Special optics "spectrally preselects" the incoming light to illuminate correspondent filter arrays. Thus, efficiency increase is achieved, and the filters transmit more light. (c) Photo of illuminated FP filter arrays (with scale bar of $200 \mu \mathrm{m}$, in gray).

In principle, there are various methods to achieve a spectral preselection, e.g., employing micro prisms, Köster prisms or dichroic beam splitters [88], which significantly differ in their complexity and spatial expansion. A suitable method ensures an optimized compromise between efficiency enhancement and minimum increase in complexity.

A very simple approach of the preselection concept can be realized using multiple dichroic longpass beam splitters arranged successively in a row and aligned to the filter arrays for the respective spectral subbands [89]. Figure 9a shows a photo of the manufactured preselection module superimposed with the schematic beam path. At first, the incoming light, provided by an optical fiber and collimated by appropriate optics, hits a deflecting prism which aligns the ray bundle with respect to the subsequent optical components. In more detail, the prism is equipped with a shortpass dichroic layer acting as a beam splitter that reflects longer wavelengths and transmits shorter wavelengths. The dichroic filter serves to separate the light that falls within the sensitivity range of the subsequent filter arrays from the wavelengths outside the stopband. In our case, wavelengths longer than $491 \mathrm{~nm}$ are reflected, which comprises all spectral ranges of the following FP filter arrays with dissimilar DBRs (521-571 nm, 576-630 nm, and 628-685 nm). Each of these applied dichroic splitter elements have a longpass characteristic. These beam splitters reflect the desired wavelength range toward the suitable filter array and transmit the residual wavelengths used for the subsequent splitters and filter arrays. The last element is also a dichroic mirror that transmits all wavelengths longer than the upper limit of the corresponding stopband.

The preselection module with the integrated FP filter arrays is combined to a mechanical adapter that allows for a connection to a CCD camera. Figure $9 \mathrm{~b}$ shows a photo of the front side of the preselection module and the mechanical adapter with all optical elements clearly visible. The comparison with the 1 Euro coin substantiates the compactness of the module $\left(17.5 \times 17.5 \times 7.8 \mathrm{~mm}^{3}\right)$. Figure $9 \mathrm{c}$ demonstrates the back side of the module with FP filter arrays. Figure $9 \mathrm{~d}$ shows a photograph of the final measurement setup with the closed module connected to a CCD camera and fed by the input light delivered by an optical fiber. With this approach, an efficiency enhancement by a factor larger than four compared to the used reference system $[11,89]$ was experimentally demonstrated.

As already mentioned, the example presented serves exclusively as a proof-of-concept. A sensor that is simultaneously optimized in terms of compactness, efficiency and spectral properties can be achieved through a joint, tailored development of detector, filter arrays and preselection setup. Although the introduction of preselection module moderately increases the overall effort for the spectral sensor, a good compromise between efficiency enhancement and increase in complexity is achieved. 


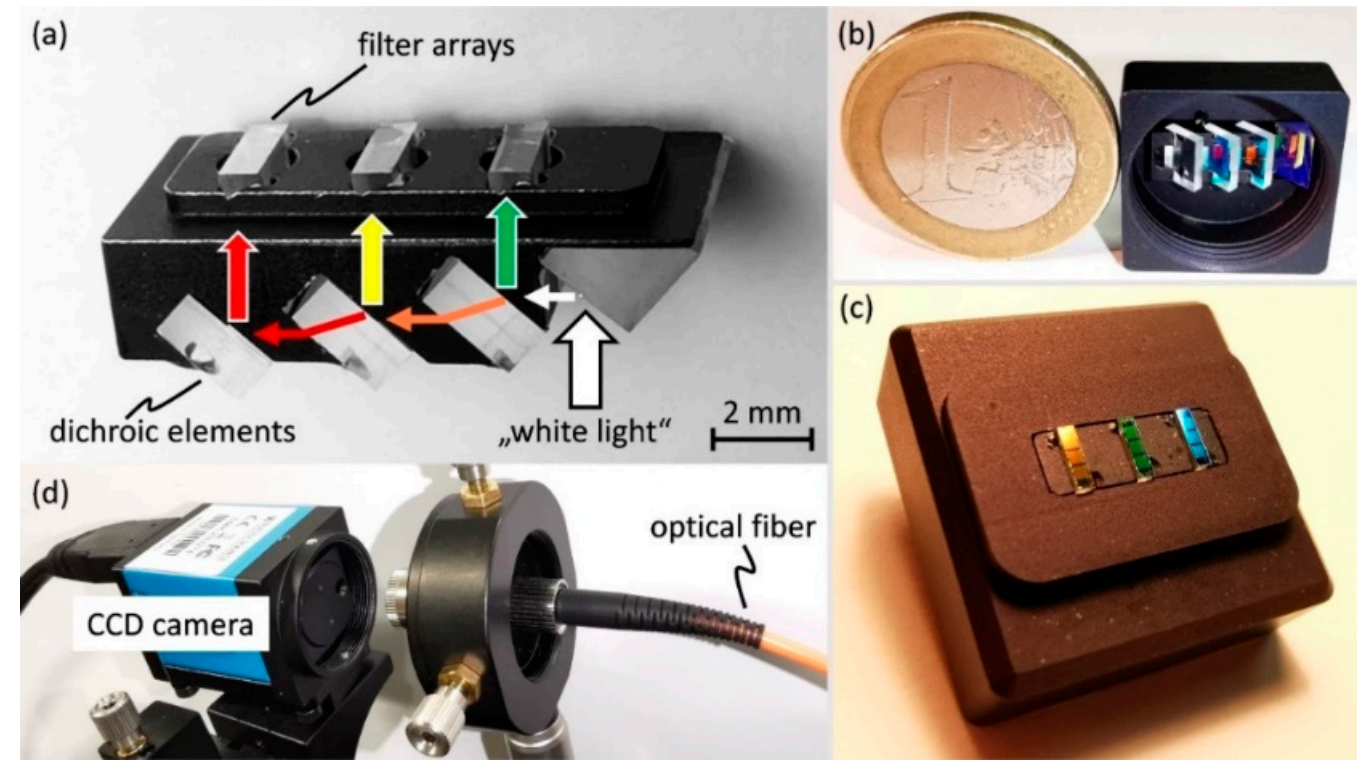

Figure 9. Efficiency increased module comprising dichroic beam splitters and FP filter arrays. (a) Photo of the preselection module with schematic beam path. $(\mathbf{b}, \mathbf{c})$ Photos of the module integrated in a housing. (b) Front side of the module displayed together with a coin (1EUR ) for size comparison. (c) Back side of the module, where the three glass substrates carrying FP filter arrays are visible. (d) Measurement setup: closed module connected to a CCD camera is illuminated by an optical fiber.

\section{Static FP Filter Arrays for the NIR: Fabrication and Characterization}

The near-infrared (NIR) spectral range is very interesting for sensing, e.g., for chemical analytics. This range was also considered in our previous paper [11] and is repeated briefly in this review. For the NIR spectral range, the FP filter arrays were designed in the 1.4-1.5 $\mu \mathrm{m}$ wavelength range, and 9.5 periods of $\mathrm{Si}_{3} \mathrm{~N}_{4} / \mathrm{SiO}_{2}$ were deposited by PECVD for the top and bottom DBR at $120{ }^{\circ} \mathrm{C}$ temperature. This avoids degradation of the polymeric cavity layer due to higher process temperatures. The mr-NIL210 resist was used to obtain cavity heights much larger than those used in Sections 3 and 4 fabricated for the VIS spectral range. Each filter element here is $40 \times 40 \mu \mathrm{m}^{2}$ in lateral dimensions, and the vertical height of the imprinted cavities ranges between 365 and $530 \mathrm{~nm}$. The experimental characterization [11] reveals high maximum transmission values up to $90 \%$ and average transmissions well above $70 \%$. The smallest FWHM $(4.7 \mathrm{~nm})$ is achieved at $1450 \mathrm{~nm}$. This provides an FP filter resolution $\lambda / \Delta \lambda$ of 300 , defined as filter transmission wavelength divided by the FWHM. Again, a comparison of experimental and simulated FWHMs had been performed. The description of the theoretical model calculations and the discussion of the FWHM variations were already described in Section 4.2. The surface roughness of layered heterostructures on the very thick resist mr-UVCur06 was investigated by an atomic force microscopy (AFM), revealing rms-values of $<4 \mathrm{~nm}$ for a single layer and $<8 \mathrm{~nm}$ for a double layer. This agrees with our FWHM observations since in layered heterostructures, this measured surface roughness is embedded in the heterostructure and transformed into interface roughness.

Finally, the influence of temperature changes on the structure and heat distribution is discussed. Having more interfaces in the heterostructure will shrink the overall thermal conductivity vertical to the interfaces. For more details see [36,90]. A good heat transfer to the heat sink is desirable since the ensemble of filter line wavelengths will shift with changing temperature. The dominating effect in the shifts results from the temperature dependence of the refractive indices of all involved materials. A much smaller effect is the influence of thermal expansion of all the layers in vertical direction. Such temperature changes for interferometric sensing application can be substantially suppressed either by implementation of Peltier elements that are commonly used in fiber optical communication 
systems, or using temperature sensors and data processing software to execute correction of temperature shifts, since this information will be captured in any case by the arrays of spectrally neighboring filters with a wide spectral span. Figures of spectra and FWHM can be found in [11].

Contrary to the static FP filter arrays in Sections 3-7, the MEMS tunable FP filters in the following sections are considered to have a single air-gap (Section 8) or multiple air-gaps (Section 9).

\section{MEMS Tunable FP Filters with a Single Air-Gap for the VIS and NIR Spectral Range}

In the range 300-1100 $\mathrm{nm}$, Si enables cheap and reliable detectors which can be integrated to powerful arrays in CCD or CMOS photodetector technology. Due to different designs, e.g., different channel geometries, they have a different spectral sensitivity. For sensorics in VIS spectral range, the combination of Si as detector material and static FP filter arrays (Sections 4 and 5) is a good and cheap solution. Therefore, MEMS tunable FP sensors in the VIS range are not as urgently required as in the NIR spectral range.

However, CMOS photodetector arrays or CCD arrays based on Silicon cannot be applied for the NIR spectral range of $>1.2 \mu \mathrm{m}$, since Si becomes transparent and is no longer sensitive for light in that range. Therefore, detector arrays such as InGaAs have to be used for the NIR instead. Considering the aspect of cost reduction of IR spectrometers, MEMS tunable spectral sensors are very attractive in the NIR applications. A large spectral range of $1.15-1.8 \mu \mathrm{m}$ (650 $\mathrm{nm}$ span) can be spanned using only three InGaAs photodiodes (an array of three photodiodes) and three related MEMS tunable filters that are neighbored in the spectrum. If each MEMS filter can be tuned over $220 \mathrm{~nm}$, three of them are enough to cover $650 \mathrm{~nm}$, with overlap. In the literature, various MEMS tunable filter designs were demonstrated in different material systems [38-58]. However, this review focuses on two MEMS concepts, namely the actuation of a single or several air-gaps.

Figure 10 depicts a MEMS tunable FP filter including a single air-gap. The lower DBR is directly connected to the substrate (not shown), which means that it remains flat and unactuated. The electrostatic actuation is displacing only the top DBR and varies the air-gap cavity height. All the incident wavelengths are reflected, except those which are able to constitute a standing wave in the cavity, and only this standing wave is allowed to transmit the filter. As already mentioned above, the following FP condition is only an approximation: The cavity height is a multiple of half of the wavelength in the cavity medium. Details on why it is only an approximation are given in Section 10.

However, this design has some disadvantages: (i) limited stopband widths and (ii) the rather bulky and stiff DBR stacks which require relatively long suspensions. The required large membrane displacements to allow large tuning ranges are only possible with such extended suspensions. If semiconductor DBRs (e.g., GaAs/AlAs) are used, the most elegant option is to n- and p-dope each of the two DBRs and to use electrostatic tuning by varying the applied voltage, as shown in Figure 10. Increasing the tuning voltage leads to a decreasing air-gap and decreasing filter wavelength (blue-shift).

If dielectric DBRs $\left(\mathrm{SiO}_{2} / \mathrm{Si}_{3} \mathrm{~N}_{4}\right.$ or $\left.\mathrm{SiO}_{2} / \mathrm{TiO}_{2}\right)$ are used, electrostatic actuation can also be applied, but this requires the specific definition of additional electrode layers since the light transmissive part of the filter must stay metal-free. In case dielectric mirrors thermal tuning is preferred, thin-film heaters are defined only on the suspensions. Increasing the tuning current through the thin-film heaters leads to increase of air-gap, thus increasing filter wavelength (red-shift). 

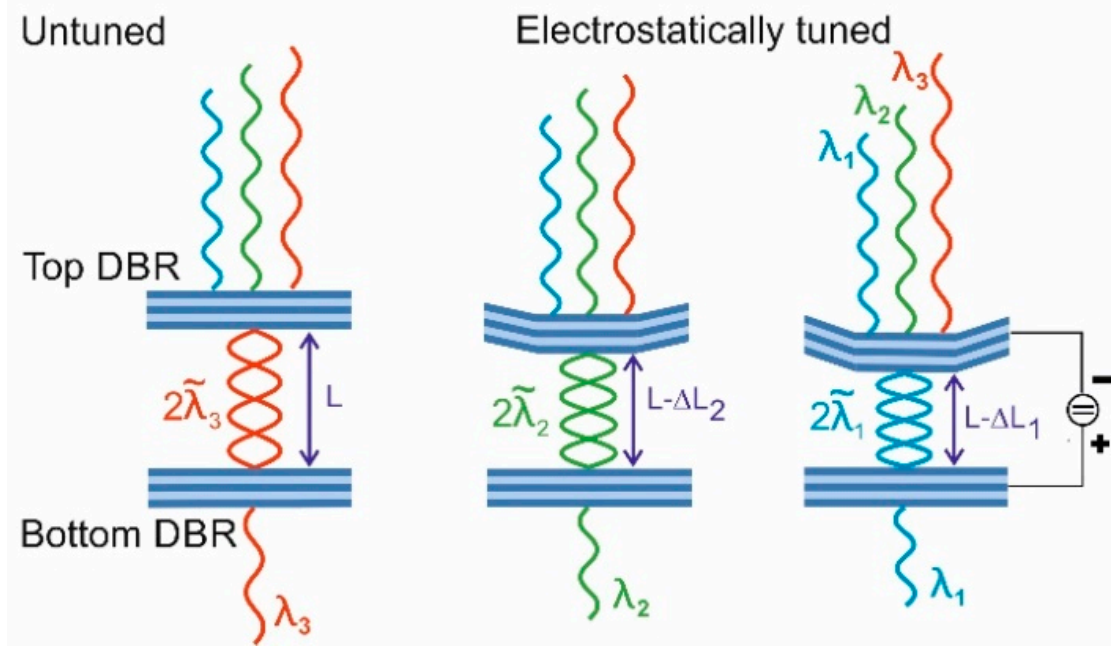

Figure 10. Single air-gap MEMS tunable FP filters with four times half-a-wavelength cavity $(4 \cdot \lambda / 2)$ and semiconductor DBRs.

Figure 11a depicts a cross section of a single air-gap FP filter with two $\mathrm{SiO}_{2} / \mathrm{Si}_{3} \mathrm{~N}_{4}$ DBRs, each with 12 periods of quarter-wave layers. The white part in between the two DBRs is the air-gap cavity with a thickness of $L=800 \mathrm{~nm}$. The absolute value of the electrical field (black line), shown as an overlay, is depicted on this multilayer structure. This visualizes the standing fundamental mode inside the interferometer for an air-gap cavity of $L=800 \mathrm{~nm}$ thickness. The electrical field clearly visualizes that all dielectric layers in the DBRs are quarter-wave layers. For the same cavity thickness of $L=800 \mathrm{~nm}$, the corresponding reflectance spectrum is displayed in Figure 11b (also in black color). Since the mirrors are dielectric and not conductive, thermal MEMS tuning is applied here. Figure $11 \mathrm{~b}$ displays corresponding reflectance spectra for different air-gap cavities of width L. In Figure 11b, a red-shift is observed with increasing tuning current, in accordance to electrostatic MEMS tuning methodology.
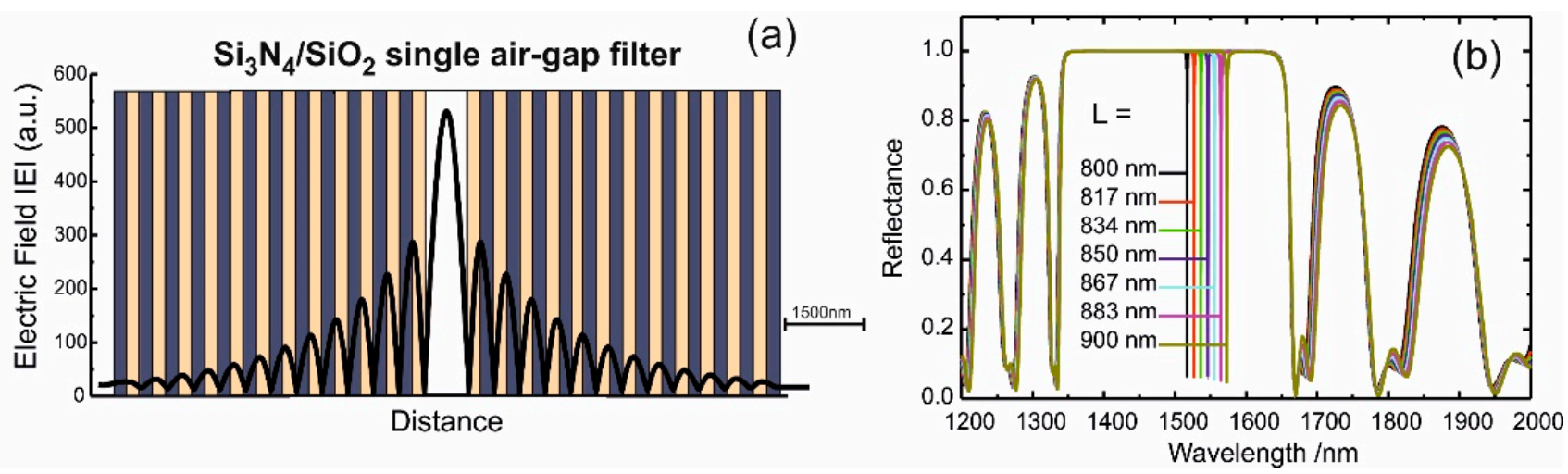

Figure 11. MEMS tunable FP filters with half-a-wavelength cavity and $\mathrm{SiO}_{2} / \mathrm{Si}_{3} \mathrm{~N}_{4}$ DBRs. (a) Cross section of the cavity embedded by dielectric DBRs together with the absolute value of the standing electrical field (black profile). (b) Corresponding reflectance spectra for different air-gap cavity widths $L$ addressed by MEMS tuning.

9. MEMS Tunable FP Filter Sensors in the NIR Range with Multiple Air-Gaps: Methodology, Simulations, Fabrication and Characterization

The abovementioned disadvantages resulting from thick DBR layer stacks and limited stopband widths can be overcome in the second airgap-based option. The second option uses multiple air-gap FP filters and is visualized in Figure 12. The cross section of the multiple air-gap FP filter is shown in Figure 12a, in which the larger central air-gap has 
the width $L$. In the DBRs, the blue InP layers are three-quarter-wave in optical thickness, and the white air-gaps in between are quarter-wave layers. This can be clearly seen from the absolute value of the standing electrical field, displayed in black. Figure $12 \mathrm{~b}$ depicts corresponding reflectance spectra for different central air-gap widths $L$ addressed by MEMS tuning. A blue-shift is observed with increasing tuning voltage, in accordance to electrostatic MEMS tuning methodology.
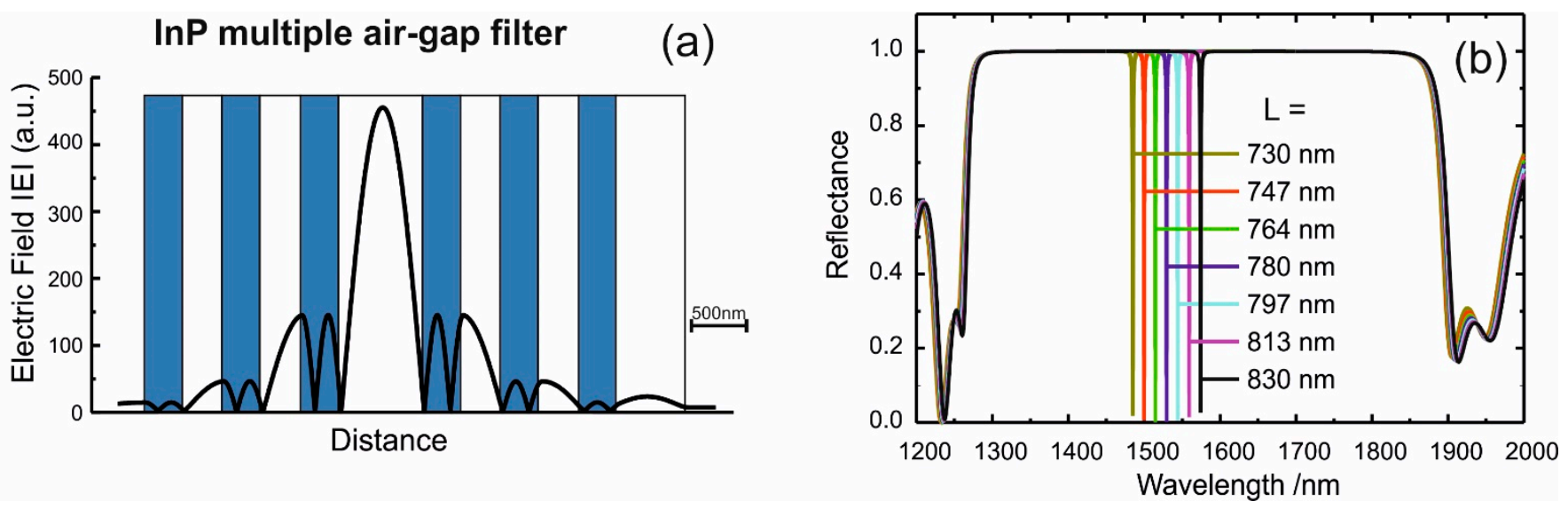

Figure 12. MEMS tunable InP multiple air-gap FP filter with half-a-wavelength central cavity. (a) Cross section of an InP multiple air-gap FP filter including the larger central air-gap of width $L$. The absolute value of the standing electrical field is displayed in black. (b) Corresponding reflectance spectra for different central air-gap widths $L$, which is obtained by MEMS tuning. In this case only $L$ is varied, all the $\lambda / 4$ air-gaps remain unchanged.

This multiple air-gap design enables large tuning ranges with a slim design and the resulting wide stopband widths, thus overcoming the disadvantages mentioned for the single air-gap design (Figure 10). Actuation of the mirrors and hence varying the cavity length is accomplished by p-doping the top DBR, n-doping the bottom DBR and applying a reverse bias. Including us, four groups have investigated vertical-cavity tunable filters based on micromachined InP/air-gap DBR using different approaches [42,45-57]. Each DBR consists of $3 \lambda_{\operatorname{InP}} / 4 \mathrm{InP}$ membranes and two $\lambda / 4$ air-gaps. The left part of Figure 13 shows a top view on an InP filter element with four supporting posts, four suspensions and the central top membrane. The right part of Figure 13 displays a cross section which is orientated according to the white broken line (left). Within the membrane and suspension posts, InGaAs served as sacrificial layers and is replaced by air upon removal. However, InGaAs within the supporting posts still remains as substantial layers.

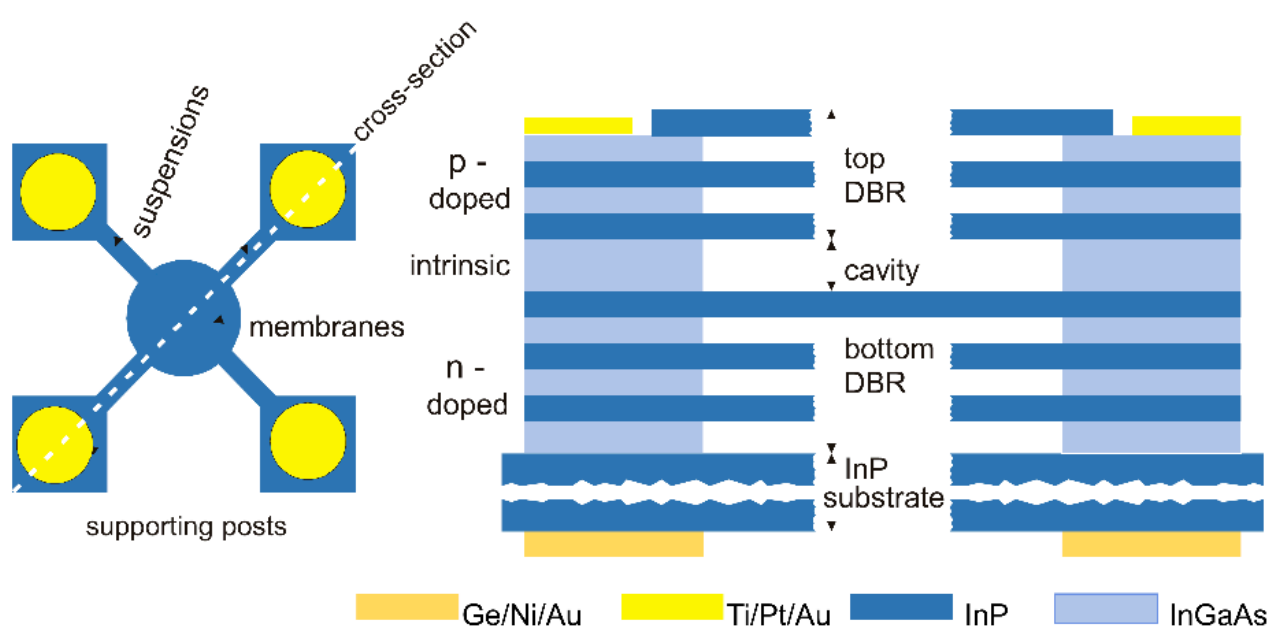

Figure 13. (Left) top view on an InP filter element showing four supporting posts with four contact 
pads (yellow), four suspensions and the top membrane (the topmost one out of the six membranes). The orientation of cross section on the right is indicated by the white broken line. (Right) cross section of the MEMS multilayer structure. InGaAs exists only inside the supporting posts. Between the suspensions and central membranes, InGaAs was serving as a sacrificial layer and had been selectively removed and replaced by air. The bottom contacts are shown in orange.

Doping of the DBRs considerably increases the tuning efficiency. Charging capabilities of metals is larger than that of semiconductors. The higher the doping level of semiconductors, the closer the semiconductor resembling a metal. Therefore, doped semiconductors are more suitable for electrostatic tuning than undoped. Reverse biasing is only shifting charges, and the current through the supporting posts is very small due to both very small areas and reverse biasing.

Metal organic chemical vapor epitaxy (MOCVD) is applied to grow the required latticematched multi heterostructure on [100] n-InP substrates: $\lambda_{\text {air }} / 4$ InGaAs layers alternating in the stack with $3 \lambda_{I n P} / 4 \mathrm{InP}$ membranes. The $\lambda_{\text {air }} / 4 \mathrm{InGaAs}$ layers act as sacrificial layers and are eventually etched, transforming into air-gaps. The top DBR located above the air-gap cavity is p-doped, and the bottom DBR layers located below the air-gap cavity is n-doped. First, the contacts are defined by lithography, evaporation and lift-off directly on the epitaxial structure. Then, $\mathrm{Si}_{3} \mathrm{~N}_{4}$ is deposited by PECVD, and lithography and reactive ion etching ( $5.1 \mathrm{sccm} \mathrm{Ar}, 3.5 \mathrm{sccm}$ CHF3, $6.7 \mathrm{~Pa}, 100 \mathrm{~W}$ ) is used to generate the $\mathrm{Si}_{3} \mathrm{~N}_{4}$ etch mask. Only the uncovered parts of the semiconductor surface are dry-etched in vertical direction using reactive ion etching $\left(20 \mathrm{sccm} \mathrm{CH}_{4}, 70 \mathrm{sccm} \mathrm{H}_{2}, 4.7 \mathrm{~Pa}, 200 \mathrm{~W}\right)$ to achieve an etch depth down to $\sim 5.5 \mu \mathrm{m}$. Use of a $\mathrm{Si}_{3} \mathrm{~N}_{4}$ mask instead of a resist mask reveals improved selectivity in the dry-etching process and avoids polymer deposition which is highly unsought on the side walls of the mesa. This process is non-selective and provides vertical side walls, and sharp edges. Then, the etch mask is removed by wet-chemical etching $\left(\mathrm{HF} / \mathrm{H}_{2} \mathrm{O}\right)$, producing pure semiconductor mesa. The supporting posts are masked by a protective layer to avoid underetching. To underetch the InP membranes, $\mathrm{FeCl}_{3} / \mathrm{H}_{2} \mathrm{O}$ is used to selectively remove the InGaAs sacrificial layers with an excellent selectivity of about 1000. The precise value depends on doping and temperature. This provides very smooth semiconductor/air interfaces. An etching time of 35 minutes at a temperature of $21{ }^{\circ} \mathrm{C}$ is used. It is important that the membranes and suspensions are underetched completely to ensure free vertical motion. The final structure after critical point drying and removal of the protection mask is depicted in Figure 13. A total of 500 filter elements were fabricated simultaneously on a wafer area of $1 \mathrm{~cm}^{2}$. The chance was used to fabricate multiple design variants differing in suspension lengths and membrane diameters on the same wafer. The filter variants have membrane diameters of $15-40 \mu \mathrm{m}$ with three or four suspensions of 10-80 $\mu \mathrm{m}$ length and $10 \mu \mathrm{m}$ width connecting the membrane to the supporting posts. Straight or bent suspensions and circular or square membranes were implemented.

Figure 14 displays the scanning electron microscope (SEM) images of filters with four suspensions. The optical quality of the two surfaces of each membrane is guaranteed by the high quality of the epitaxial heterointerfaces which is preserved in the selective chemical etching step, providing very low optical roughness. Our surface micromachining fabrication process demands no micro-mounting since the entire structure is fabricated in a sequence of process steps. Furthermore, a monolithic integration is obtained within the GaInAsP/InP material system, allowing the integration of photodiodes and verticalcavity surface emitting lasers. The central, light gray circular areas in Figure $14 a-c$ show unprotected regions to allow underetching of membranes and suspensions. The dark gray areas outside of the circular areas show protected regions. Figure $14 \mathrm{~d}$ provides a closer look on the suspension region, especially through the wider central air-gap that allows a view through the structure to the ground behind, marked with "*". The ground level in front is marked with "**". A single suspension has a width of $10 \mu \mathrm{m}$ (light blue). The top DBR consists of the layers 1,2, and 3 and the bottom DBR is made of layers 4,5 and 6. At the central area, a view on top of suspension 4 is possible (marked with number 4 in white). 
(a)

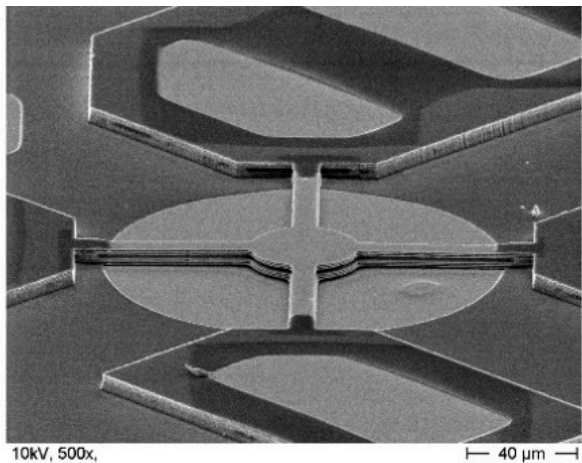

(c)

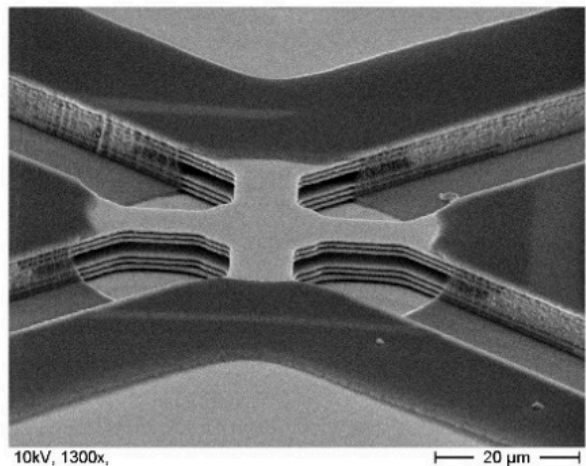

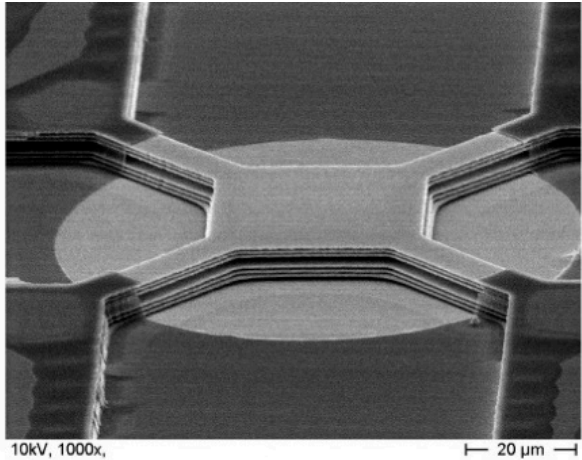

(b)

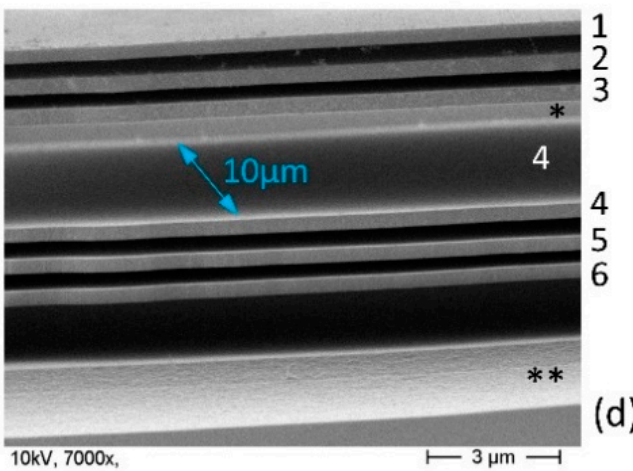

Figure 14. SEM micrographs of InP/InGaAs multiple air-gap MEMS tunable FP filters. Each DBR consists of three InP layers and two air-gaps. (a) $60 \mu \mathrm{m}$ long suspensions and a circular membrane with $40 \mu \mathrm{m}$ diameter. (b) Square membranes. (c) Short suspensions and small membranes. (d) Details of the suspension region. It allows a view through the structure to the ground behind marked with "*". The ground level in front is marked with "***".

Figure 15a displays an SEM micrograph top view of an InP multiple FP filter prior to the wet-chemical under-etching. The light gray circular region allows the etching solution to remove the sacrificial layers therein. The dark gray region outside prevents the underlying structures from being etched. Figure $15 \mathrm{~b}$ shows a detailed close-up image of the suspension and membrane region, allowing a view through the central air-gap. Figure $15 \mathrm{c}$ displays the filter line wavelength as a function of actuation voltage, and the corresponding reflectance spectra is shown in the inset. A very wide tuning range of $221 \mathrm{~nm}$ [56] for a variation of the actuation voltage of $0-28 \mathrm{~V}$ is measured for our MEMS filters. To the best of our knowledge, this is the largest tuning range measured for any $\mathrm{InP} /$ multiple air-gap DBR-based verticalcavity filter. The FWHM is very narrow (about $1 \mathrm{~nm}$ ) at lower actuation voltages; however, it broadens with increasing actuation voltage due to buckling of the membranes.

MEMS tunable filters were also fabricated using nanoimprint technology, successfully reducing the sacrificial layer to zero. A methodology proposed by Cheng et al. [91] was selected and adapted using selective curing in silica template. It was modified and transformed into our hybrid SCIL stamps [92]. Using UV-blocking metal layers in the otherwise transparent stamps, areas with non-cured nanoimprint resist could be generated. Therefore, cured hard resist remains only in the area of supporting posts, while other areas with non-cured resist show non-existent residual layers during resist removal. Being able to avoid residual layers was an important step forward for our SCIL 3D nanoimprint technology [92].

Figure 16 presents a $3 \mathrm{D}$ perspective view of the whole sensor attached to a fiber. A MEMS tunable FP filter is integrated with an InGaAs photodiode. The MEMS filter and the photodiode were grown within the same MOCVD epitaxial run on InP substrate. 

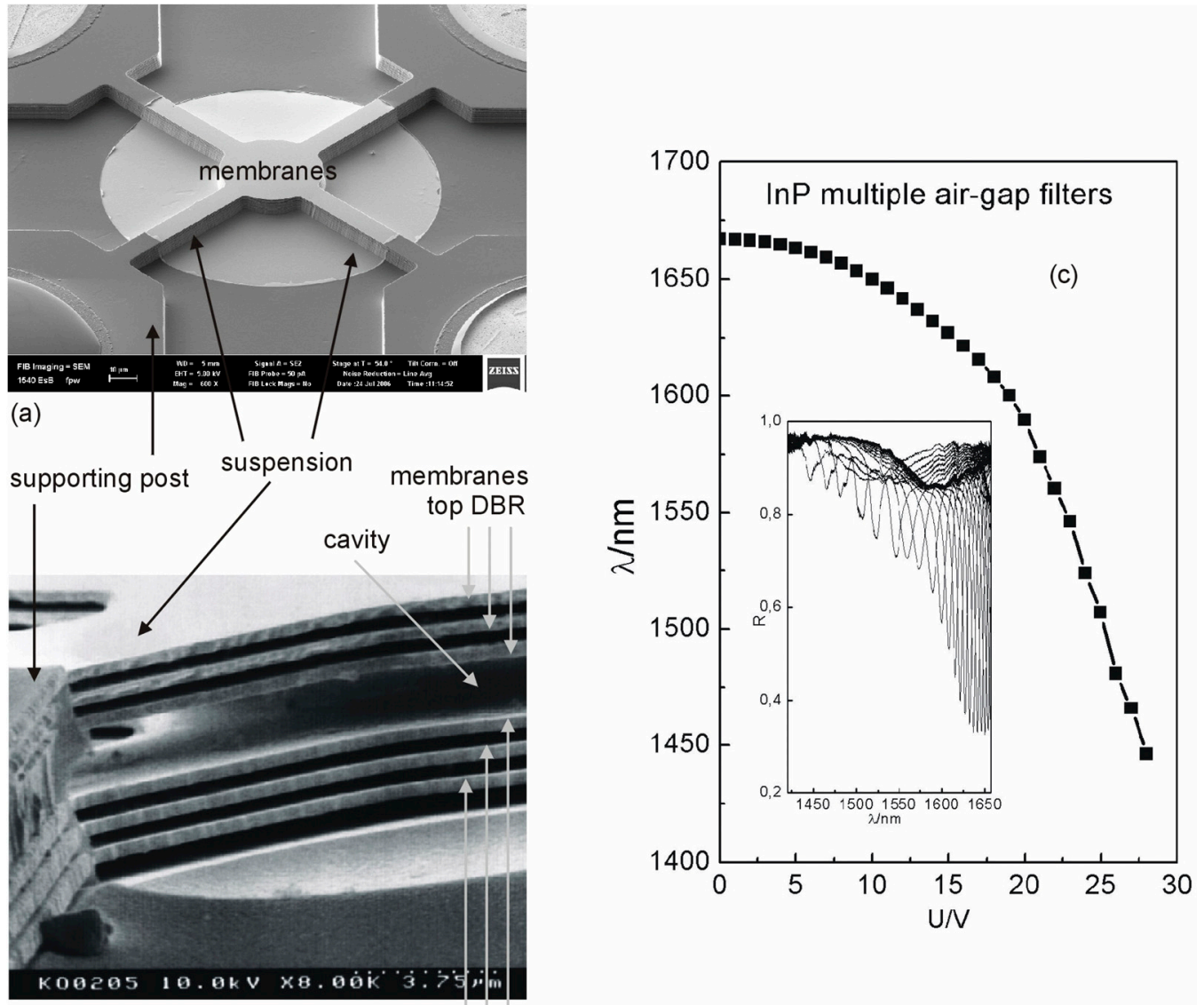

(b)

membranes bottom DBR

Figure 15. (a) SEM image in tilted top view on an InP multiple air-gap MEMS tunable FP filter with InP/InGaAs supporting posts with contacts on top; (b) SEM image in tilted side view, enabling a look through the central airgap between the suspensions; (c) Tuning characteristic showing the filter peak wavelength as a function of the actuation voltage. A selection of experimental reflection spectra $R(\lambda)$ is displayed in the inset. Various spectra are shown for different actuation voltages $U$. Reprinted with permission from ref. [11]. Copyright 2021 MDPI.

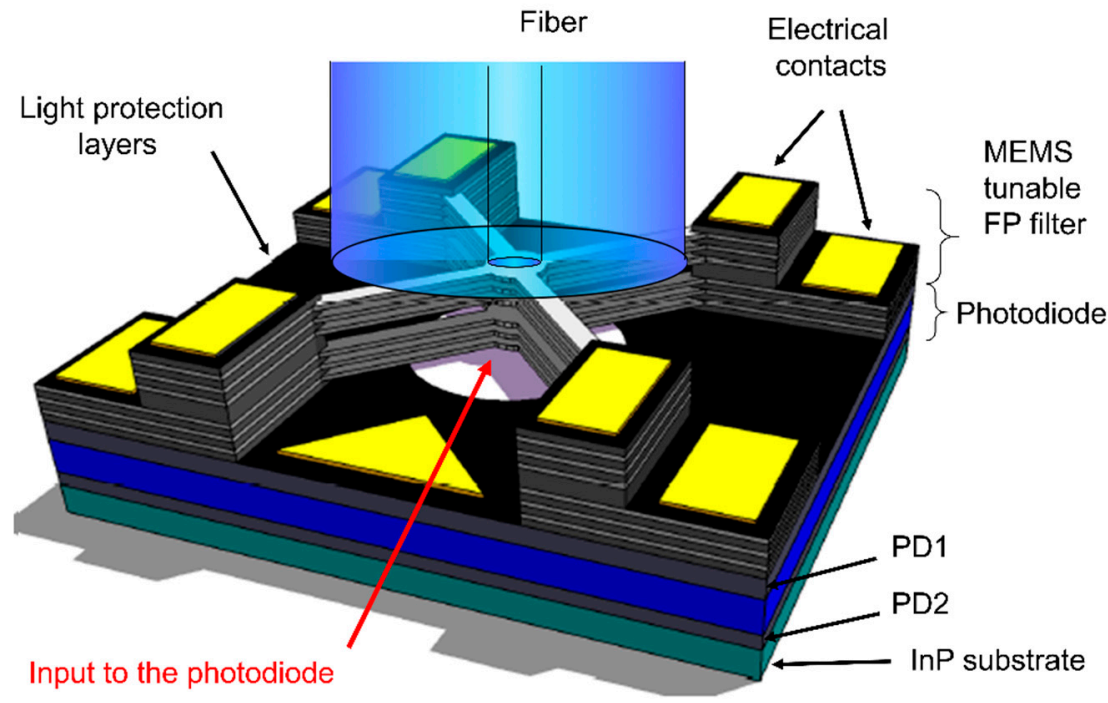

Figure 16. Interferometric sensor for the NIR spectral range, consisting of a MEMS tunable filter and 
a photodiode. The actuation voltage will be applied between the rectangular and triangular contact pads. The photodiode is biased via the two highly doped semiconductor layers PD1 and PD4, and the sensor signal is also picked off between PD1 (triangular contact pad) and PD4 (substrate contact). To allow light incidence only on the white area, the rest of the PD top is covered by light protection layers. The rectangular contact pads correspond to the round contact pads in Figures 13 and 15, as well as to the rectangular contact pads in Figure 14.

The InP material system enabling monolithic integration with InGaAs photodiodes is not only beneficial for NIR sensorics, but also very favorable for receiver systems to be used for NIR wavelengths tailored for telecommunication requirements of low fiber dispersion and low fiber absorption. Our lab demonstrator including MEMS tunable FP filters and InGaAs photodiodes in monolithic integration was working well in the laboratory practice. In addition, a packaged MEMS tunable filter with two fiber pigtails was demonstrated using the InP/multiple air-gap material system.

\section{Limits of Semiconductor and Dielectric Material Systems for MEMS-Based Sensorics: Geometry, FWHM, Tuning and Stopband Width}

In this section, further conceptional facts and details are presented as a continuation to Sections 8 and 9. A separate Section 10 was chosen for this purpose to avoid overloading and interruption of the common thought in the previous two sections.

The first question is, how do the membranes of the top and bottom DBR move under electrostatic actuation? Figure 17a shows the InP multiple air-gap FP filter without applied voltage (unactuated), and Figure $17 \mathrm{~b}$ displays the real situation when an actuation voltage is applied (actuated state). Because of applied voltage, charges are shifted. The charges accumulate mainly at the inner surfaces of the two central membranes. Therefore, the two central membranes are mainly actuated. Consequently, the outer membranes stay nearly uncharged and remain nearly unactuated. The outer membranes remain nearly unaffected due to shielding effects. Figure 17c displays a hypothetic situation, in which all the membranes of the top DBR are actuated in the same way and the whole bottom DBR moves in unity.

In Figure $18 \mathrm{a}, \mathrm{b}$ the difference in tunability between the two cases shown in Figure $17 \mathrm{~b}, \mathrm{c}$ are investigated. Performing detailed theoretical model calculations using the TransferMatrix method the respective spectra, tuning ranges and FWHM have been calculated. The comparison starts from the same unactuated situation (blue spectrum) and studies different actuation states $\Delta L=50 \mathrm{~nm}, 100 \mathrm{~nm}$ and $150 \mathrm{~nm}$ (the remaining central air-gap is $L-\Delta L$ ). The spectra corresponding to these tuning conditions are displayed in different colors. The hypothetic case is shown in Figure 17c actuating all membranes, and the realistic cases is displayed in Figure 17b actuating only the central membranes.

For the two cases, the wavelength tuning $\Delta \lambda$ of the filter line is plotted as a function of the central air-gap height difference $\Delta L$ in Figure 19. Moving all the membranes of each DBR leads to a higher MEMS tunability.

The tuning efficiency is $\Delta \lambda / \Delta L=0.92$ if all membranes are actuated, and it is otherwise $\Delta \lambda / \Delta L=0.8$ if only central membranes are actuated. In comparison, the obtained tuning efficiency in Figure $11 \mathrm{~b}$ is much smaller, i.e., $\Delta \lambda / \Delta L=0.56$ for $\mathrm{SiO}_{2} / \mathrm{Si}_{3} \mathrm{~N}_{4} \mathrm{DBRs}$. The multiple air-gap InP methodology is not only much better in MEMS tuning but also much more compact. The InP multiple air-gap FP filter reveals the smallest extension of the whole multilayer unit in the literature, with exception to photonic crystal membranes. Please note that the length scales of the two FP filters in Figures 11a and 12a are not identical. 

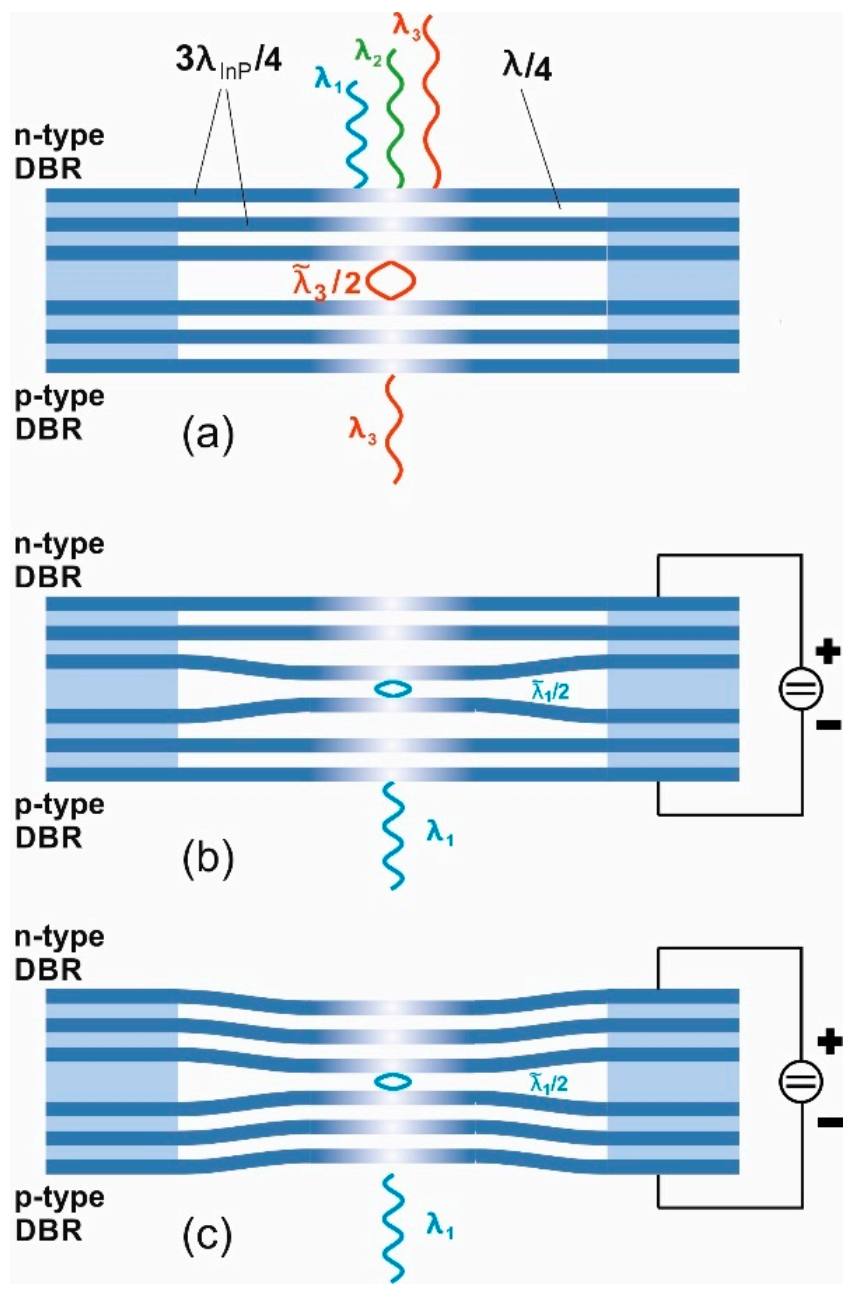

Figure 17. Schematic of MEMS tunable FP filters including multiple air-gaps and membranes and suspensions made of InP. The supporting posts are built of the complete InP/InGaAs multilayer stack, as grown by epitaxy. (a) Unactuated state; (b) Actuated state where only the two central membranes of the DBRs are actuated; (c) Actuation of all membranes (i.e., actuating the whole two DBR stacks). This tuning configuration is shown in Figures 12 and 18a. The meaning of $\widetilde{\lambda}$ used in $(\mathbf{a}-\mathbf{c})$ is explained hereinafter in this section.
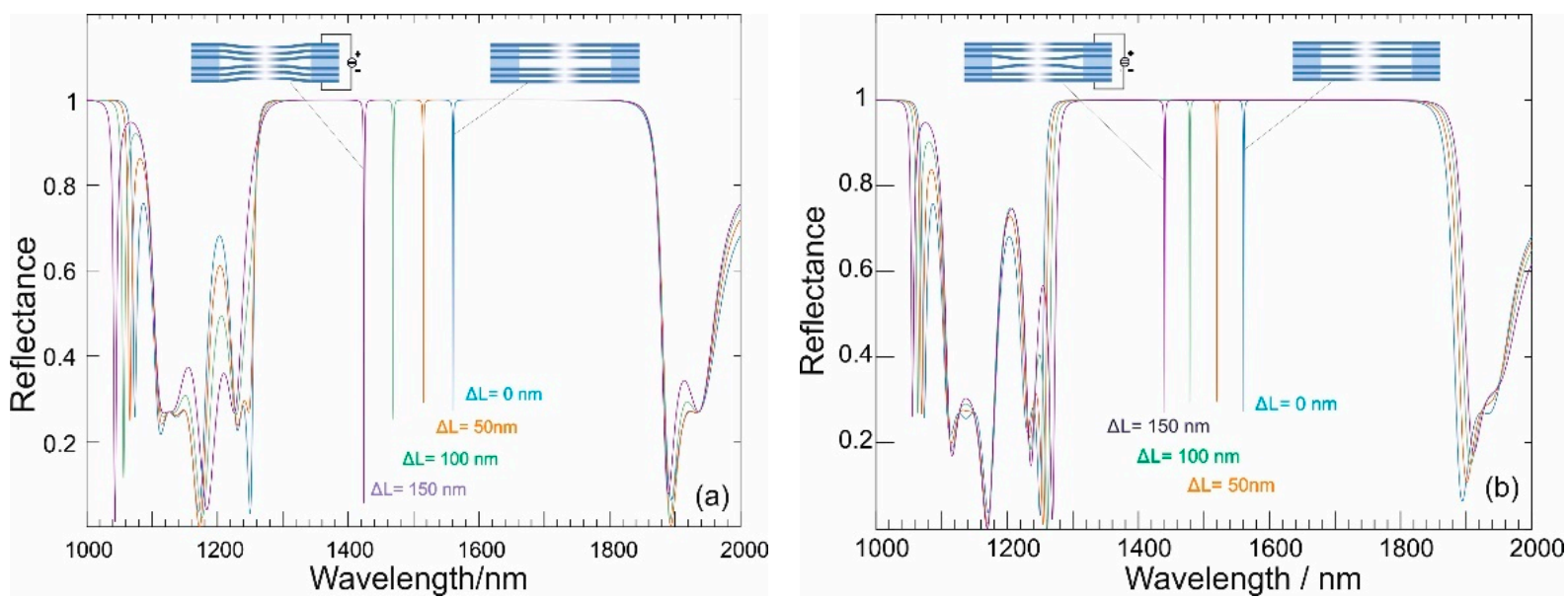

Figure 18. Electrostatic MEMS tuning of InP multiple air-gap filters, (a) actuating all membranes of the DBRs and (b) actuating only the two central membranes. 


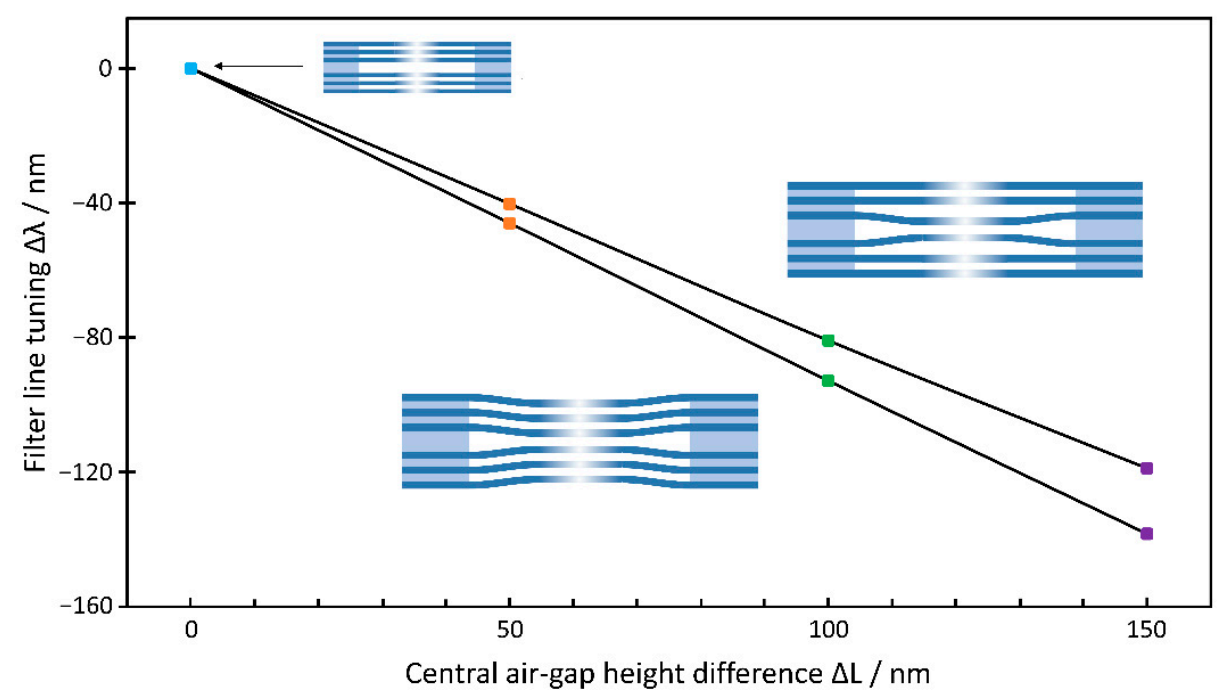

Figure 19. Electrostatic MEMS tuning of InP multiple air-gap filters with two different cases: Actuating all membranes of the DBRs (lower curve) and actuating only the two central membranes (upper curve). The colors of the symbols correspond to those used in Figure 18.

It is also worth noting that many misunderstandings are related to the often and commonly used term "half-a-wavelength cavity". The FP condition for two metallic mirrors states reads: the cavity length $L$ is a multiple of half of the wavelength in the medium. Figures 11a, 12a and 17a show half-a-wavelength cavities. Since the electromagnetic wave penetrates into the DBR mirrors, the figures show a feature which looks like half a wavelength inside the mode (Eigenfunction), but that is an effective wavelength $\widetilde{\lambda}$ and not the half vacuum wavelength. It is not the same, and it depends on the DBR mirrors. During the tuning of the length $L$, the nodes are no longer located at the interfaces between the layers as shown in Figures 11a and 12a. Considering the insufficient idea of half of the vacuum wavelength inside the cavity, the tuning efficiency $\Delta \lambda / \Delta L$ should be 1 , independent of the materials of the DBR. A detailed explanation can be found in one of our doctorate dissertations [93].

The FWHM are nearly identical in both cases compared in Figure 18. A possible explanation could be that the FWHM is dominantly related to the number of periods. Since the number of periods is equal in both cases in Figure 18, the FWHM remain nearly identical.

Figure 19 reveals that the difference in tuning of the two cases is $13 \%$. The wavelength tuning is dominantly related to changes in the airgap thicknesses. Therefore, the differences between the two cases are more pronounced in tunability rather than in the FWHM. However, quantifying $13 \%$, the difference is still relatively small. This might be due to the fact that the mode shapes adapt not only to the changes in the central air-gap but also to the changes of the two other air-gaps which are located next to the central air-gap. For the complex localization of nodes and interfaces during the tuning, please refer to [93].

Next, the limits of FWHM are studied for multiple air-gap InP filters and displayed in Figure 20. Theoretical model calculations based on the transfer-matrix method are used to simulate the spectra of InP multiple air-gap FP filter lines. Measured data for the spectral InP absorption coefficient is given as $\alpha_{I n P}=3.43 \mathrm{~cm}^{-1}$. The DBRs consist of $357 \mathrm{~nm}$ InP $\left(3 \lambda_{I n P} / 4\right)$ and $3675 \mathrm{~nm}$ air $\left(\lambda_{\text {air }} / 4\right)$ which are embedding the central air-gap of $L=815$ $\mathrm{nm}\left(0.53 \lambda_{\text {air }}\right)$. The red curve is simulated for DBRs with three InP membranes, resulting in FWHM of $1.01 \mathrm{~nm}$ and dip reflectance of 0.00043 at a dip wavelength of $1562.995 \mathrm{~nm}$. The black curve is simulated for DBRs with four InP membranes, resulting in FWHM of $0.112 \mathrm{~nm}$ and dip reflectance of 0.028 at a dip wavelength of $1562.906 \mathrm{~nm}$. 


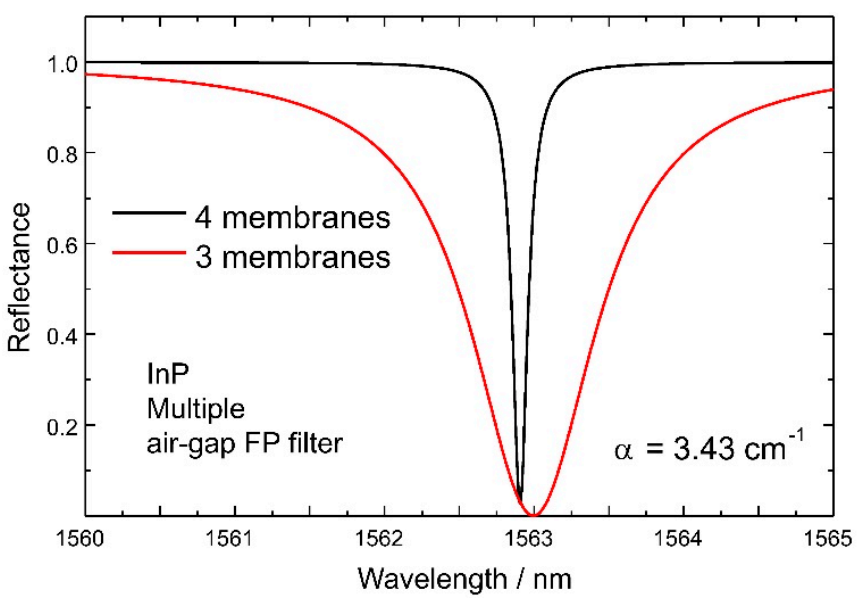

Figure 20. Transfer-matrix model calculations of InP multiple air-gap FP filter line spectra with DBRs including 3 and $4 \mathrm{InP}$ membranes each.

These low values could be confirmed experimentally with four InP membranes, in which a FWHM of $0.1 \mathrm{~nm}$ was measured at $1.55 \mu \mathrm{m}$. However, such a low value was only measured once from a single sample. Such linewidth broadening can only be avoided if the non-bending (buckling) of the central membrane areas is completely absent. Notwithstanding, the simulations and measurements showed what is possible. The optical resolution of the FP filter methodology is predicted to be around 15,000 in the best case.

Next, the maximum potential DBR characteristics are reviewed and shown in Figure 21. Applying transfer-matrix model calculations, transmission and reflectance spectra are calculated for different numbers of periods $p$ and the appropriate material absorption coefficients $\alpha$. The spectral variation of absorption $\alpha=\alpha(\lambda)$ is taken from experimental results. In the inset of Figure 21a, the spectral reflectance is shown for a $\mathrm{Si}_{3} \mathrm{~N}_{4} / \mathrm{SiO}_{2} \mathrm{DBR}(p=12$, $\lambda_{i} / 4$ layers, $\alpha=0$ ). The maximum spectral reflectivity $R_{\max }$ for $\lambda=1.55 \mu \mathrm{m}$ (see arrow) is extracted from all the spectra which were calculated for $\mathrm{Si}_{3} \mathrm{~N}_{4} / \mathrm{SiO}_{2}$ and $\mathrm{InP} /$ air-gap DBRs. $R_{\max }$ for $\lambda=1.55 \mu \mathrm{m}$ is plotted in Figure 21a as a function of the number of periods $p$. Numerous spectra ranging the absorption loss $\alpha=0,0.1,0.3,1,3,10,20$, and $100 \mathrm{~cm}^{-1}$ were calculated for $\mathrm{Si}_{3} \mathrm{~N}_{4} / \mathrm{SiO}_{2}$ DBR's. Please note that the values of $\alpha$ are determined by the technological fabrication and the appropriate process parameters. However, for ultra-pure semiconductor material, the optical loss dominantly is related only to the band structure in defect-free crystalline material of high quality. Therefore, low material loss can be guaranteed, and it is understood well in these crystalline materials. Figure 21a reveals that $R_{\max }$ of a DBR strongly grows with increasing $p$, but it saturates for higher values of $p$. The level of $R_{\max }$ saturation is strongly decreased with growing loss. As already mentioned, the extended spectral region of high reflectivity (i.e., the spectral plateau in the center, as shown in the inset of Figure 21c) represents the stop-band.

According to Figure 21a, the dielectric $\mathrm{Si}_{3} \mathrm{~N}_{4} / \mathrm{SiO}_{2}$ system $(\lambda=1.55 \mu \mathrm{m}, \Delta n=0.47$, $n_{\mathrm{Si} 3 \mathrm{~N} 4}=1.94, \alpha_{\mathrm{Si} 3 \mathrm{~N} 4}=\alpha_{\mathrm{SiO} 2}$ both varied, $n_{\mathrm{SiO} 2}=1.47$ ) yields $R_{\max }>0.998$ already for $p \geq 12$ if loss is neglected. For $\alpha_{\mathrm{Si} 3 \mathrm{~N} 4}=\alpha_{\mathrm{SiO} 2}=15 \mathrm{~cm}^{-1}$, a reflectivity of $99.8 \%$ is exceeded for $p>14$. In contrast, $99.8 \%$ reflectivity cannot be reached for $\alpha_{\mathrm{Si} 3 \mathrm{~N} 4}=\alpha_{\mathrm{SiO} 2}=20 \mathrm{~cm}^{-1}$ since $R_{\max }$ saturates at $R_{\max , \text { sat }}=0.997$. Please note that $\Delta n$ is much larger for $\mathrm{InP} /$ air-gap structures on InP substrates which was the motivation to use and study this system $(\lambda=1.55 \mu \mathrm{m}$, $\Delta n=2.167, n_{I n P}=3.167, n_{\text {Air }}=1, \alpha_{I n P}=3.4 \mathrm{~cm}^{-1}$ ) which provides a maximum reflectance $R_{\max }$ of 0.9998 for $p=4$ and yields $R_{\max }$ exceeding $99.8 \%$ if $p \geq 3$. To share further values, three $\mathrm{InP}$ /air-gap membranes embedded in air on both sides (in contrast to the previous structure no InP substrate is considered) reveal $R_{\max }=0.99993$ for $p=4.5$ and 0.9996 for $p=3.5$. This demonstrates that using an air "substrate" instead of an InP substrate reveals a much larger $R_{\max }$ already for smaller $p$ due to a larger refractive index contrast at the 
exterior ends of the DBR mirror. In summary, type and presence of a substrate strongly influence the optical data, especially for smaller number of periods $p$.
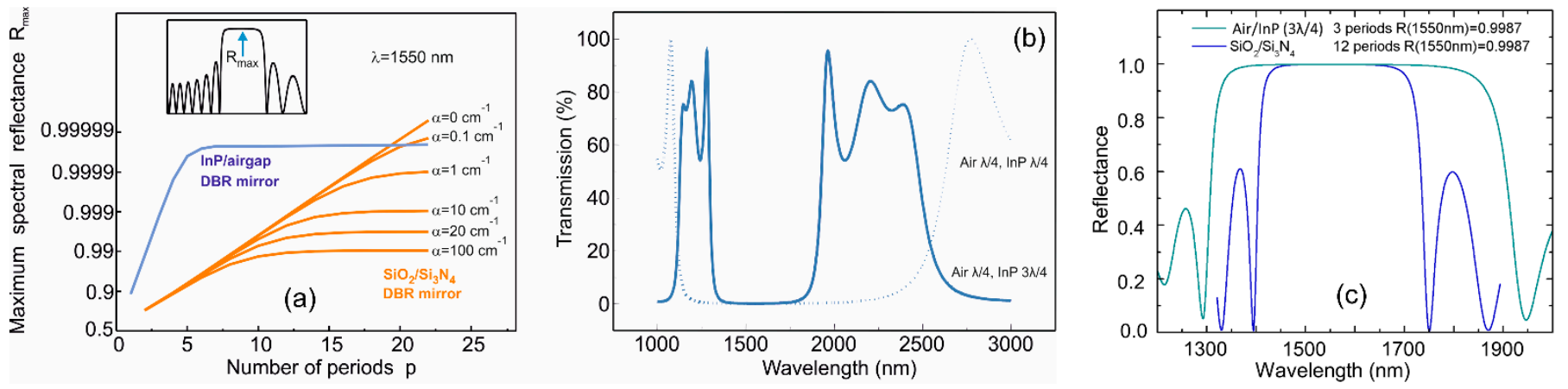

Figure 21. (a) Calculated maximum spectral reflectance $R_{\max }$ for $\mathrm{Si}_{3} \mathrm{~N}_{4} / \mathrm{SiO}_{2}$ DBRs (orange) as a function of the number of periods $p$ showing the absorption coefficient $\alpha$ as a variation parameter. In the case of the InP multiple air-gap DBRs (blue), $\alpha_{\text {InP }}=3.43 \mathrm{~cm}^{-1}$. The inset displays the calculated reflectance spectrum indicating the spectral position of the maximum spectral reflectance $R_{\max }$. (b) Transmission spectra for InP multiple air-gap DBRs. The dotted line shows a DBR structure including thinner InP layers $\left(\lambda_{\text {InP }} / 4\right)$ revealing a huge stopband of $1500 \mathrm{~nm}$. The full line shows a structure including thicker InP layers $\left(3 \lambda_{\text {InP }} / 4\right)$ revealing a smaller stopband of $500 \mathrm{~nm}$. (c) Reflectance spectra for $\mathrm{Si}_{3} \mathrm{~N}_{4} / \mathrm{SiO}_{2} \mathrm{DBRs}$ and InP multiple airgap DBRs.

In Figure 21b, the benefit is seen resulting from a very large refractive index contrast between 1 (air) and 3.167 (InP) existing between the two DBR materials for $\lambda=1.55 \mu \mathrm{m}$. This high contrast enables very large stopbands: $500 \mathrm{~nm}$ for the combination of $\lambda_{\text {air }} / 4$ with $3 \lambda_{\operatorname{InP}} / 4$, and $1500 \mathrm{~nm}$ for the combination of $\lambda_{\text {air }} / 4$ with $\lambda_{\operatorname{InP}} / 4$. Even the $3 \lambda_{\operatorname{InP}} / 4 \operatorname{InP}$ membranes is already sufficient to produce the stopband width exceeding the values of $\mathrm{Si}_{3} \mathrm{~N}_{4} / \mathrm{SiO}_{2}$ by far (Figure 21c).

Notwithstanding, none of the four groups fabricating InP multiple air-gap DBRs has achieved to successfully fabricate $\lambda_{I n P} / 4$ suspended InP membranes up until now. The main issue lies in the breaking of the suspensions and membranes during the drying process after selectively removing the InGaAs sacrificial layers. For this reason, the dotted profile in Figure $21 \mathrm{~b}$ remains a dream. Hopefully, improved future fabrication technologies with solvents implying less turbulences and improved drying processes can enable further progress on this challenging field. Thus, the full line in Figure $21 \mathrm{~b}$ dealing with $3 \lambda_{\operatorname{InP}} / 4$ thick membranes is the state-of-the-art in the present times.

Due to the arsenic carry-over in the epitaxial growth of the ... InP/InGaAs/InP ... heterostructure layer, the two interfaces are not identical. After finishing the InGaAs growth and switching to InP, traces of arsenic are still in the MOCVD reactor and are incorporated into the InP close to the interface. In contrast: after finishing the InP growth and switching to InGaAs, no arsenic can be carried back into the already finished InP layer. This arsenic carry-over leads to small stress gradients and subsequently buckling (bending) of the membranes and suspensions after removal of the InGaAs sacrificial layers. Completely flat membranes were obtained in our epitaxial growth after additional doping of arsenic in the opposite interface, and thus creating symmetric structures: arsenic-doped $\mathrm{InP} /$ undoped InP/arsenic-doped InP.

\section{Further Concepts for Miniaturization Based on Plasmonics, Ring Resonators, Quantum Dots, Spatial Heterodyning, and Photonic Crystals on Fiber Tips and in MEMS Membranes}

Beside the widely applied concepts of interferometric sensors beeing discussed in the previous sections, further options for miniaturization of sensors exist. In some of them, DBRs or even a complete FP filter are replaced by a single layer with 1D or 2D periodic patterns. Other concepts make use of different interaction effects such as plasmonic 
resonances or Raman scattering. In the following section, a short overview to examples of all these approaches is given.

\subsection{Sensors Based on Photonic Crystals in MEMS Membranes}

As already covered in the introduction, guided mode resonances allow either broador narrowband resonances by coupling a wave in and out of a slab waveguide, and then superimpose this resonant mode with the directly reflected and transmitted continuous mode, respectively. The result is a Fano shaped resonance line in the spectrum, where bandwidth and line shape are given by the coupling condition. The basic concept of coupling resonant and continuous modes is shown In Figure 22a, and an example of a single InP MEMS membrane with a square lattice of elliptical holes can be seen in Figure 22b.
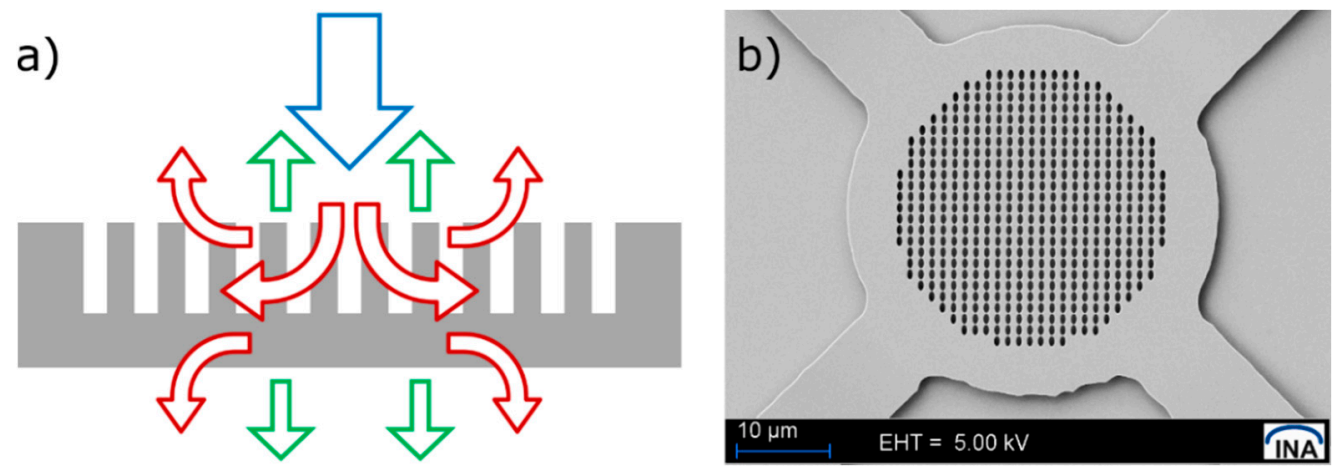

Figure 22. (a) Principle of a guided mode resonance. The incident wave (blue) is partly transmitted and reflected in form of continuous modes (green) and partly couples in and out the slab in form of a resonant mode (red). Superposition of both parts leads to the typical Fano line shape. (b) A MEMS membrane with elliptical air holes as base elements in a 2D square lattice, breaking the symmetry and leading to polarization dependent reflection and transmission.

Zobenica et al. [77] applied two parallel membranes including photonic crystals (PCs) and quantum dots (QDs), which were coupled evanescently. In this case, a single DBR is replaced by two PC layers. This idea was shown first in $[62,73]$. The graph in the third column from the right-hand side in Figure 3 depicts the design of the MEMS PC membrane. These devices reveal a very low FWHM of $0.08 \mathrm{~nm}$ for central wavelength at $1319 \mu \mathrm{m}$. The spectral filter lines could be tuned in the experiments across $30 \mathrm{~nm}$ by electrostatic MEMS actuation. However, it is challenging to adjust position and size of the QD for distinct wavelengths.

Using PC structures with MEMS tunable narrowband filters, polarization selectivity can be additionally implemented. This maintains the compactness of MEMS devices by introducing holes with pronounced elliptical symmetry in the PC structure of the top membrane in the top DBR [64]. Here, most commonly guided mode resonance structures [63,66] or structural birefringence [67] are applied. In both cases, the selective behavior of the filter device regarding electric field orientation of the incident wave is based on disturbing the $90^{\circ}$-symmetry by either line gratings or introducing elliptical base elements in a 2D PC.

\subsection{Nano-Optical Sensor Concepts}

A spectroscopic MEMS sensor using the excitation of surface plasmon-polariton (SPP) resonance on a cantilever was reported by Oshita et al. [68]. The device is fabricated from a SOI wafer, and a metallic grating is applied on top to provide the excitation condition for SPPs. In operation, the cantilever is oscillating at resonance frequency close to $400 \mathrm{~Hz}$ and provides the largest angular stroke. As the coupling condition for SPP to the metallic grating is strongly angle dependent, the signal recorded for half an oscillation period can be evaluated to get the spectral information. In the cited work, a spectral scanning range of $300 \mathrm{~nm}$ was achieved with a FWHM of approximately $10 \mathrm{~nm}$ or more. The spectral range 
is mainly limited by the properties of the materials and the grating. Further data are given in Figure 3 in the second column from the right-hand side.

Another approach, presented by Faraji-Dana et al., applies tailored dispersion properties of diffractive elements using a folded beam path in a thin glass plate where light is reflected at three specifically designed metasurfaces [94]. The authors showed a spectrometer covering $100 \mathrm{~nm}$ spectral range with $1.2 \mathrm{~nm}$ resolution and device volume of $7 \mathrm{~mm}^{3}$.

\subsection{Sensors with Links to Telecom Devices}

Strong ties exist between spectroscopic sensors and devices used in multiplexing systems of optical telecommunication systems. In both cases, the goal is to analyze spectrally encoded information, but the focus for telecommunication is mostly on spectral linewidth, whereas in spectroscopy a broad spectral range is often required as well. Transfer of concepts from one field to the other is generally beneficial, and the most common device types such as FP or AWG are frequently found in both fields. Two examples of spectroscopic sensors derived from telecom concepts are presented in the following.

Filters with extremely small FWHM were demonstrated by Li et al. [78] and are included in Figure 1 at the right-hand side. This device is based on a fiber Bragg grating (FBG) with linear chirp. Local heating at a defined position of the FBG introduces a change in refractive index and therefore a phase shift in the grating period. Phase shifts in periodic patterns lead to resonant conditions and high transmission for a certain wavelength. Such behavior can also be understood by looking at the cavity of a DBR FP filter in the same way. A $\lambda / 2$ layer in a periodic sequence of $\lambda / 4$ layers represents a phase shift in the layer structure as well. The device presented by Li et al. is tunable for $16.5 \mathrm{~nm}$ in the spectral range around $1.55 \mu \mathrm{m}$, with a FWHM of only $0.007 \mathrm{~nm}$.

The application of ring resonators is another path to achieve sub-nm spectral resolution. These devices are commonly used in telecom systems and are known to have very high $Q$ factors of more than 100,000, but also small free spectral ranges as they work in a high order. Nitkowski et al. showed such a spectrometer integrated to a microfluidic chip [95]. From the same group, an improved device combining a ring resonator, a diffraction grating and a waveguide array was presented later, with $0.05 \mathrm{~nm}$ FWHM in the near IR spectral range and a footprint of $2 \mathrm{~mm}^{2}$ [96]. Smooth sidewalls of the stripe waveguides and accurate dimensions of the ring structures are crucial, but the planar technology is well understood and commercially applied in the telecom field for many years already.

\subsection{Sensors with Computational Signal Evaluation}

For several recently developed spectrometer devices, such as the plasmonic MEMS sensor shown by Oshiita et al., computational post-processing of the acquired data are an essential part of the sensing principle. A very interesting and compact example in this direction is the nanowire-based spectrometer of Yang et al. [97]. The II-VI semiconductor of the nanowire is grown with gradually varying composition along the length of the wire, leading to a varying band gap. By choosing the appropriate base material, several spectral ranges can be addressed. An array of small electrodes is contacted to the nanowire and allows the measurement of a locally generated photocurrent. Evaluation of the signals is based on a pre-calibrated response function and an algorithm to extract the spectral data. The authors show results over a spectral range of $130 \mathrm{~nm}$ with FWHM of approximately $8 \mathrm{~nm}$ in the visible range. The device size is determined by the length of the nanowire and the dimension of the electrode array, both being on the order of $100 \mu \mathrm{m}$ or less.

\subsection{Sensors on the Fiber Tip}

A highly efficient way to integrate optical measurement methods with fiber systems is to use the optical fiber itself as sensor. Beside the small size given by the fiber diameter, the typical advantages of fiber optics, namely its mechanical flexibility and immunity to electromagnetic interference, make this type of sensors interesting for measurements in difficult locations and harsh environments. However, most of the sensors are not tunable 
or require additional electrical components at the opposite end of the fiber, thus increasing the overall size of the system again. Furthermore, the fabrication of fiber-based sensors can be quite challenging and will be addressed at the end of this discussion. Interaction with light waves can occur inside the fiber based on structures such as fiber Bragg gratings or on evaluation of scattering effects. In the following overview, a different approach where the sensor is fabricated on the tip of an optical fiber will be covered. In such a configuration, interaction with light waves occurs locally at the tip, and the fiber is merely used for guiding the wave to a detector.

As previously introduced, guided mode resonances (GMR) in 1D or 2D photonic slab waveguides enable very compact filter elements on MEMS devices. Tailoring the coupling strength between resonant and continuous mode allows for narrowband spectral properties, whereas breaking symmetries will lead to polarization dependent behavior. In a similar way, GMR filter elements can be implemented on the tip of optical fibers, as shown in Figure 23. A high refractive index thin film of $\mathrm{ZrO}_{2}$ was deposited by ion beam sputter deposition on the fiber tip and acts as a slab waveguide with $\mathrm{SiO}_{2}$ and air as surrounding materials. The periodic pattern, centered at the core of the single-mode fiber, was fabricated by FIB milling lithography. Nanoimprint lithography on a fiber tip is another possible fabrication method, in which the whole facet is covered and patterned in a single process step as shown by Tabassum et al. [98]. The fabrication is far simpler to implement than using sputter deposition and focused ion beam (FIB) milling, but it lacks the precise control of layer thickness or alignment to the fiber core.

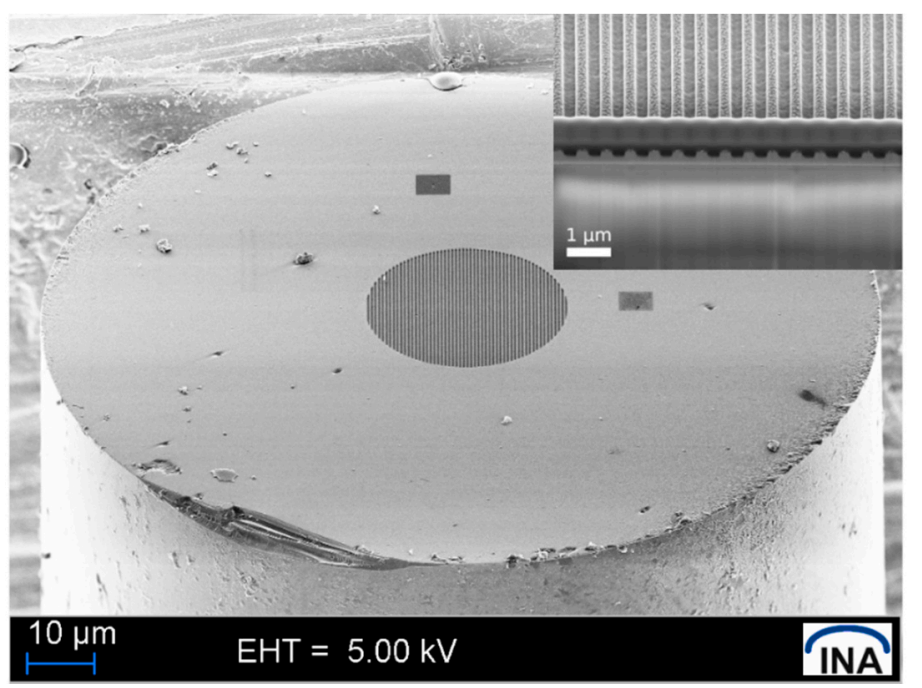

Figure 23. Tip of an optical fiber with GMR structure aligned to the core. The inset shows an FIB cross section of the $\mathrm{ZrO}_{2}$ slab waveguide layer deposited by ion beam sputter deposition and the 1D periodic pattern fabricated by FIB milling. The dark bar covering a part of the pattern is a locally deposited protection layer to avoid damage of the pattern during preparation of the cross section and to enhance contrast for imaging.

Several research groups showed FP filters on fiber tips, mainly for sensing applications in gases and liquids. Mostly, air cavities are implemented by splicing a short section of multi-mode fiber or capillary to a single-mode fiber. Ma and Wang showed that fabricating a MEMS mirror on the spliced segment by sputter coating and FIB milling and subsequent selective underetching leads to an FP with the single-mode fiber facet being the second mirror. Target application of this sensor was detection of $\mathrm{H}_{2}$ in gas atmospheres [99]. The combination of a single-mode fiber and a short photonic crystal fiber (PCF) segment with high index core and large surrounding air holes as fiber tip sensor was presented by Zhu et al. [100]. An FP cavity forms by reflections due to the mode mismatch at the splice and at the PCF to air interface and was applied to measure temperatures by tracing the optical path length variation resulting from thermal expansion and effective refractive 
index change. A similar pressure sensor fabricated of a short capillary spliced to a singlemode fiber and covered by a few-layer graphene sheet as flexible membrane and FP mirror was shown by Ma et al. [101]. Kilic et al. [102] presented an acoustic sensor working on the FP principle as well, but first they fabricated a PC mirror on a released MEMS membrane using the GMR effect with a broadband resonance. The silicon on insulator chip was then mounted with high-viscosity epoxy to the fiber tip, forming an air cavity between silicon membrane and a metal layer coated on the fiber facet. Monolithic fabrication of an FP cavity by FIB milling was reported by Alberts et al. [103] using an off-centered, partly metal coated reflector for application as refractometer in liquids.

Beside the interferometric principles for sensors, plasmonic fiber tip devices are also a viable option. Still, using surface plasmon polariton resonances is difficult due to the required excitation condition. De Maria et al. [104] showed, however, that polishing the fiber tip under the excitation angle and coating the surface with silver is a possible solution for this problem. More common is the application of localized plasmon polariton (LPP) resonance by exciting free electron oscillations in nano-sized particles or structures $[105,106]$. Since excitation of LPP resonances can be accomplished by free space modes, no extra preparation is required. A typical application is the enhancement of weak intensity signals in Raman spectroscopy using the local field enhancement around the metallic nano structures, known as surface enhanced Raman spectroscopy (SERS) [107]. Kostovski et al. showed that this method can be integrated into a fiber tip sensor using nanoimprint lithography and subsequent deposition of a metal layer [108]. Probes based on nanoscale fiber tips and with additional metallization [109] are applied widely for scanning near field optical microscopy. Plasmonic effects or optical antennas are used to characterize optical properties which are not accessible to other methods. For example, Burresi et al [110] report on probing the magnetic field of an optical wave by applying a metallic split ring aperture at a tapered fiber tip.

Further implementations of sensor on fiber tips exist. In some, the optical fiber is only used as signal path, whereas the interaction with a sample is based on non-optical effects. The fiber tip atomic force microscope, presented by Iannuzzi et al. is an example from this group of sensors [111]. As mentioned, the technology required for the fabrication of fiber tip devices can be challenging. This is due to the small and not perfectly flat surface of a fiber facet, which makes resist coating or lithography quite difficult. Nevertheless, several routes for possible processes have already been shown [112]. These include FIB milling, nanoimprint lithography, align \& shine photo lithography, two photon polymerization lithography, or mounting of chips fabricated by conventional methods.

\subsection{Spatial Heterodyne Sensors}

As a complement to the different, mainly FP-based sensor concepts discussed so far, the Spatial Heterodyne Spectrometer (SHS) is considered in the following section. The attractiveness of the SHS concept is based on the possibilities for tailoring its optical properties to specific user requirements in combination with its significant potential for miniaturization.

Essentially, the SHS is based on the configuration of a Michelson interferometer, where the mirrors in each arm are substituted by diffraction gratings [113]. The gratings are fixed in a tilted mount and do not require moving parts.

The basic working principle of SHS is schematically shown in Figure 24 (according to [114]). The incoming polychromatic wavefronts are separated by the beam splitter into two partial wavefronts, each striking on a respective grating. The orientation of the grating determines the Littrow wavelength, whose wavefront is diffracted exactly backward to the incident direction. All other wavelengths have different diffraction angles and propagation directions. At the detector, the superimposed light from both gratings is recorded. In detail, the periodicity of the interference pattern $\Lambda$ for a specific wavelength $\lambda$ depends on the half angle between intersecting wavevectors $\theta$ and the refractive index $n$ of the ambient medium. The entire recorded interferogram is composed by the contribution of all involved wavelengths. Finally, the interferogram must be converted to a conventional 
spectrum by Fourier transformation. The working principle allows very high spectral resolution for a small spectral bandwidth.

Applications for SHS range from space born projects e.g., satellite-based atmospheric temperature measurements [115] to Raman Spectroscopy of minerals [114]. Particularly impressive are the small dimensions that can be achieved. A SHS system was presented in [115], operating between $761.9 \mathrm{~nm}$ and $765.3 \mathrm{~nm}$ with a resolving power of about 8000 , and requiring only a volume of $38 \times 38 \times 27 \mathrm{~mm}^{3}$ in size. In the meantime, progress has also been made in the production technology of SHS. Yi et al. [116] reported on the fabrication procedure of monolithic interferometers for SHS systems with ultraviolet curing adhesive and commercial optical elements, which may open the door to high volume manufacturing.

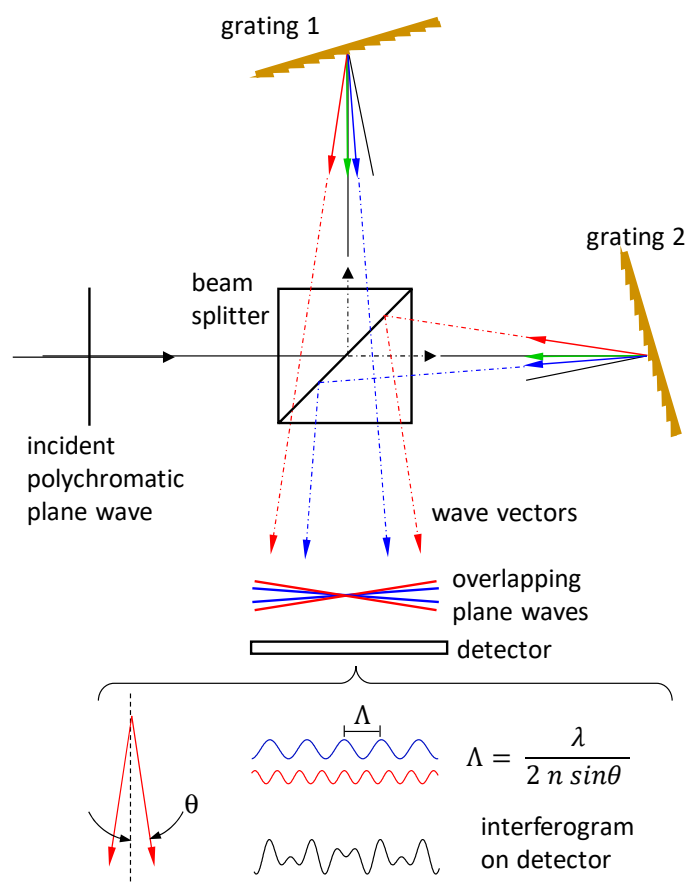

Figure 24. Illustration of the basic principle of spatial heterodyne spectroscopy (SHS).

\section{Estimation of Potential Space Requirement after Utmost Miniaturization}

Concerning grating spectrometers, the company Hamamatsu and Ibsen Photonics most probably came close to the miniaturization limits defined by such an optical resolution, which is still sufficient for some applications. In the case of our VIS and NIR FP filter arrays, the miniaturization potential in lateral direction was not used in our proof-of-principle. With $40 \times 40 \mu \mathrm{m}^{2}$ in lateral direction, the mesa is considerably very big, and therein lies a high miniaturization potential to make them smaller. In this section, the potential limits for miniaturization of all six sensor principles are estimated: AWG, static FP filter array, MEMS tunable FP filter arrays, plasmonic sensors, MEMS tunable PC filters, and chirped fiber Bragg gratings. To identify the miniaturization limits, best case scenarios (ideal conditions) and optimum vertical light incidence are considered. For the static arrangements (FP filter array, AWG), $\Delta \lambda=2 \mathrm{~nm}$ in the VIS range was chosen for the spectral step size (channel spacing) between spectrally neighboring transmission lines; and in the NIR range, a channel spacing of $\Delta \lambda=4 \mathrm{~nm}$. A spectral span of $400 \mathrm{~nm}$ in the VIS range and a spectral span of $500 \mathrm{~nm}$ in the NIR range has to be covered. Although a preliminary estimation was already performed in [11], here it is further complemented with inclusion of two additional sensor concepts and supplementary figures visualizing the lateral arrangements. 


\subsection{Static FP Filter Arrays Covering $400 \mathrm{~nm}$ in the VIS Spectral Range}

In Figure 25a, the minimum space requirements for a static FP filter array for the VIS spectral range is shown. To eliminate effects from borders within a single pixel, the lateral size of the square optically active mesa (orange) has to be approximatively $8 \lambda \times 8 \lambda$ or larger, which was derived from experiments and simulations. This means that $6 \mu \mathrm{m}$ side dimensions of the square mesa are sufficient for the wavelength range $\lambda=400-800 \mathrm{~nm}$. Choosing a spatial spacing of $4 \mu \mathrm{m}$ between the square mesa, results in a period of $10 \mu \mathrm{m}$ and reveals an area of $10 \times 10 \mu \mathrm{m}^{2}$ for each pixel. The material system $\mathrm{TiO}_{2} / \mathrm{SiO}_{2}$ for the DBRs allows stopband widths of $200 \mathrm{~nm}$, thus, 100 spectrally adjacent filter lines in each stopband are required. Two neighboring stopbands are required to span $400 \mathrm{~nm}$ (Figure 25a). To be on the safe side for mounting issues, empty frames of $50 \mu \mathrm{m}$ width surrounding both arrays are chosen. A space of $204 \times 358 \mu \mathrm{m}^{2}$ is required, resulting in a chip size of about $0.07 \mathrm{~mm}^{2}$. This is well compatible with commercial Si CCD detector arrays or Si CMOS detector arrays. All signal recording and processing electronics are located behind the layer containing the filters as depicted in Figure 25a, leading to a very compact layout.
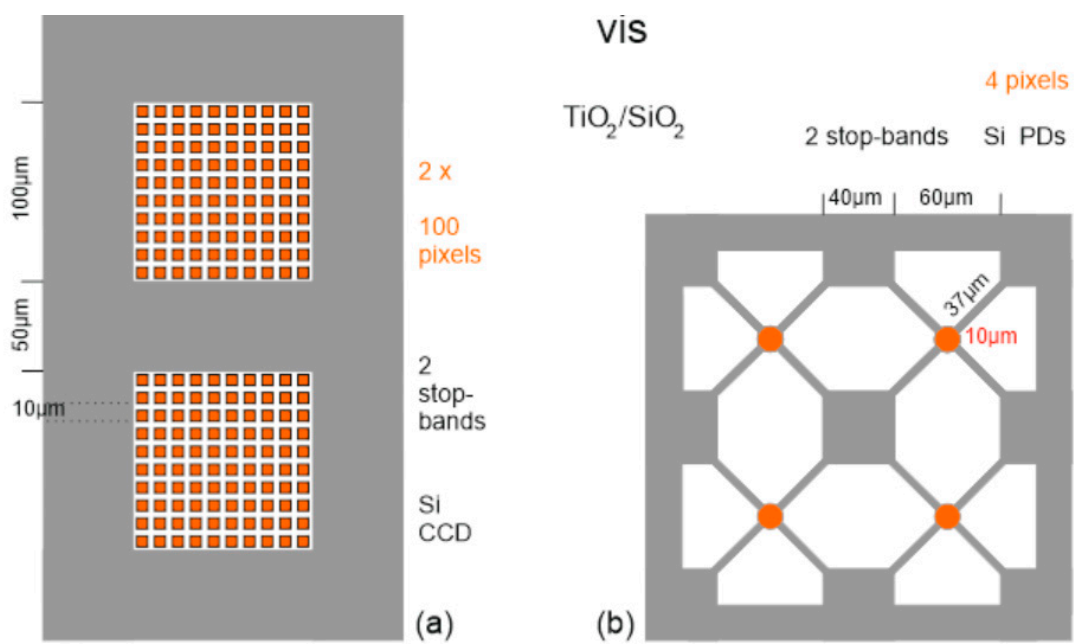

Figure 25. Space requirement to cover $400 \mathrm{~nm}$ in the VIS spectral range using two neighboring $\mathrm{TiO}_{2} / \mathrm{SiO}_{2}$ stopbands for the DBRs and PDs/CCDs in the Si material system. (a) Static FP filter array. (b) MEMS tunable FP filters.

\subsection{Static FP Filter Arrays Covering $500 \mathrm{~nm}$ in the NIR Spectral Range}

Since Si cannot be used for this purpose, other detector material such as InGaAs is required. However, photodiodes in the InGaAs material system are by far more expensive than $\mathrm{Si} C \mathrm{CD}$ or CMOS detector arrays. The company Hamamatsu offers linear InGaAs photodiode arrays which have stripe-like active areas (orange in Figure 26) of $15 \times 100 \mu \mathrm{m}^{2}$ which are followed by stripes of $10 \times 100 \mu \mathrm{m}^{2}$ as spacers (white in Figure 26). Therefore, the period of the spacer/stripe arrangement is $25 \mu \mathrm{m}$. For $\lambda=1500 \mathrm{~nm}$, this also fulfills the required $8 \lambda$ minimum pixel size. A minimum of 125 pixels are required for one $\mathrm{DBR}$ in the $\mathrm{TiO}_{2} / \mathrm{SiO}_{2}$ material system with a stopband of $500 \mathrm{~nm}$, which translates to $100 \times 3125 \mu^{2}$ (Figure 26). Considering an empty frame of $100 \mu \mathrm{m}$ width around the linear array, $300 \times 3325 \mu \mathrm{m}^{2}$ space are required, resulting in $1 \mathrm{~mm}^{2}$ chip size. Such approximated size excludes any signal processing electronics that still have to be integrated in this commercial solution. 


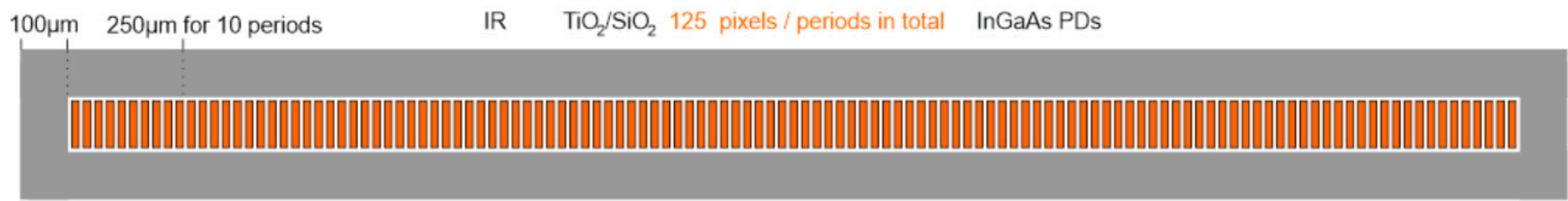

Figure 26. Space requirement to cover $500 \mathrm{~nm}$ in the NIR spectral range using commercial InGaAs PDs. Please note that this array is considerably scaled down in size compared to Figures 25 and 27.

This requires a huge amount of space. However, if tailored square photodiodes would be used, the required space would shrink considerably. However, this technology is not yet available on the market. Figure 27a displays an estimate for the required space for that case. To avoid border effects within a single pixel, the lateral size of the square optically active mesa (orange in Figure 27a) should also be approximately $8 \lambda \times 8 \lambda$ or larger, known from experiments and simulations. Thus, $13 \mu \mathrm{m}$ for both sides of the squares are sufficient for the wavelength range of $\lambda=1500 \mathrm{~nm}$. Using $4 \mu \mathrm{m}$ spatial spacing between the mesa, the period is $17 \mu \mathrm{m}$ in length. Thus, an area of $17 \times 17 \mu \mathrm{m}^{2}$ are required for each pixel. A single $\mathrm{TiO}_{2} / \mathrm{SiO}_{2} \mathrm{DBR}$ with a stopband of $500 \mathrm{~nm}$ already covers the required spectral range. Since the required 125 pixels are not practical for a rectangular array, 130 pixels are chosen (Figure 27a) and arranged in a $10 \times 13$ array. Applying empty frames of $50 \mu \mathrm{m}$ width surrounding arrays, a total of $274 \times 325 \mu \mathrm{m}^{2}$ space is required. This results in a chip size of about $0.09 \mathrm{~mm}^{2}$. For a very compact design, the complete signal processing electronics should be placed behind the sensor part as depicted in Figure 27a. However, such technology (corresponding to Si CCD or Si CMOS) has not yet been developed in the InGaAs/InP-based material system. Nevertheless, a hybrid solution with a Si CMOS chip behind the InP chip is also possible, as shown in Figure 5.
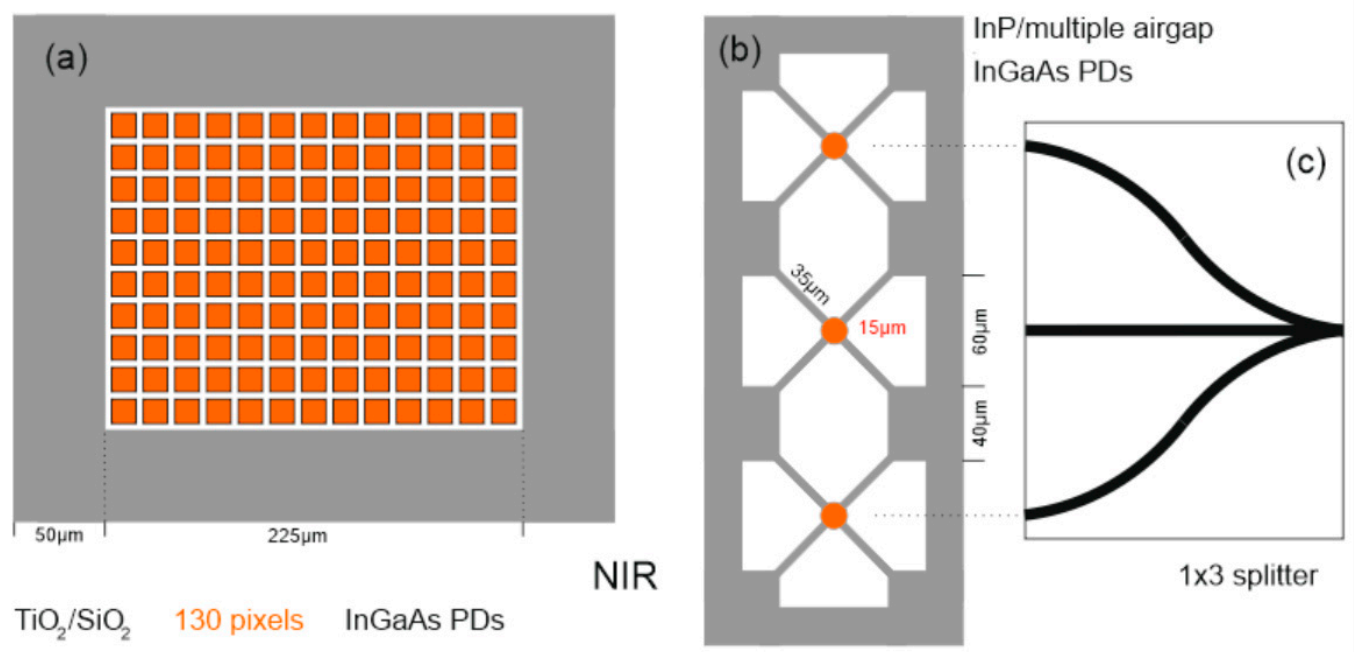

Figure 27. Space requirement to cover $500 \mathrm{~nm}$ in the NIR spectral range using tailored InGaAs PDs, not yet available on the market. (a) Static FP filter array with a single $\mathrm{TiO}_{2} / \mathrm{SiO}_{2} \mathrm{DBR}$. (b) MEMS tuneable FP filters in the InP multiple airgap system. (c) $1 \times 3$ splitter, schematically aligned to the 3 MEMS membranes.

\subsection{MEMS Tunable FP Filter Arrays Covering $500 \mathrm{~nm}$ in the NIR Spectral Range}

The InP/multiple airgap material system allows DBRs with a stopband width between $500 \mathrm{~nm}$ and $1500 \mathrm{~nm}$, depending on the InP layer thicknesses $\left(3 \lambda_{\operatorname{InP}} / 4\right.$ or $\left.\lambda_{\operatorname{InP}} / 4\right)$, respectively. Therefore, a single stopband is already enough to cover the required $500 \mathrm{~nm}$ wavelength span in the NIR spectral range. In the experiment, a tuning range of $221 \mathrm{~nm}$ was obtained. To be on the safe side, three MEMS tunable filters are used to cover the 
required $500 \mathrm{~nm}$. For our minimum space estimation, the following required elements are arranged according to Figure 27b: supporting posts spanning a lateral area of $40 \times 40 \mu^{2}$, suspensions with $35 \mu \mathrm{m}$ lengths, and circular membranes with $15 \mu \mathrm{m}$ diameters. The $15 \mu \mathrm{m}$ diameter is fully sufficient to cover the wavelength range between 1.2-1.7 $\mu \mathrm{m}$. As shown in Figure $27 \mathrm{~b}$, the central filter is sharing its supporting posts with the two neighboring filters. Applying empty frames of $20 \mu \mathrm{m}$ all around the three filters ensures that the optically active membrane (orange) is at least $50 \mu \mathrm{m}$ apart from the chip borders. This requires $340 \times 140 \mu \mathrm{m}^{2}$ space and reveals a chip size of $0.05 \mathrm{~mm}^{2}$. As demonstrated, the advantage in this concept is that the InGaAs Photodiode can be fabricated together with the MEMS filter within the same epitaxial step (monolithic integration), as depicted in Figure 16. Concerning fiber and waveguide optics, a $1 \times 3$ integrated waveguide splitter (Figure 27c) could be used to divide the light from the fiber and to guide it to the three active regions (shown in orange). Please note that the plane spanned in Figure $27 \mathrm{c}$ is perpendicular to the plane spanned in Figure 27b, and the three ends of the splitter are ending centrally to the three orange membranes. This is indicated by the two dotted lines relating Figure 27b,c. The fiber would be coupled to the right-hand side waveguide end of Figure 27c.

\subsection{MEMS Tunable FP Filter Arrays Covering $400 \mathrm{~nm}$ in the VIS Spectral Range}

The material system $\mathrm{TiO}_{2} / \mathrm{SiO}_{2}$ is chosen for the DBRs, allowing stopband widths of about $200 \mathrm{~nm}$. According to Figure 25b, the required elements are supporting posts with an area of $40 \times 40 \mu \mathrm{m}^{2}$, four suspensions with lengths of $35 \mu \mathrm{m}$, and circular membranes (orange) of $10 \mu \mathrm{m}$ diameter. This diameter is sufficient for application at $\lambda=400 \ldots 800 \mathrm{~nm}$. Please note that the dielectric material system exhibits much smaller tuning ranges due to the single air-gap MEMS in comparison to the slim InP multiple airgap system. Although they have $200 \mathrm{~nm}$ wide stopbands, four tunable FP filters (each with $100 \mathrm{~nm}$ tuning range) are required to cover the targeted $400 \mathrm{~nm}$ (Figure 25b). Neighboring filters share supporting posts which are located in between. As in Section 12.3, applying empty frames of $20 \mu \mathrm{m}$ all around the four filters ensures that the optically active membrane (orange) is at least $50 \mu \mathrm{m}$ apart from the chip borders. This requires $240 \times 240 \mu \mathrm{m}^{2}$ space and results in a chip size of $0.06 \mathrm{~mm}^{2}$. Similar to previous cases, the complete signal processing electronics is placed behind the sensor part depicted in Figure $25 \mathrm{~b}$ to make it very compact. Concerning fiber and waveguide optics, a linear arrangement of the four MEMS filters would be a better option (like in Figure 27b), and a $1 \times 4$ integrated waveguide splitter should be used to divide the light from the fiber to the four active regions (orange) as in Figure 27c.

It is important to note that in Sections 12.1-12.4, the photodetectors and the electronics are hidden behind the filters elements and not visible in Figures 25-27.

\subsection{MEMS Tunable PC Filter to Cover a Spectral Span of $500 \mathrm{~nm}$ in the NIR Range}

Zobenica et al. [77] measured a tuning range of $30 \mathrm{~nm}(1308-1338 \mathrm{~nm})$ and pointed out that there is a potential to extend it to $40 \mathrm{~nm}$. A wavelength span of $500 \mathrm{~nm}$ can be covered by combining 15 spectrally neighbored filters, supporting posts of $15 \times 50 \mu \mathrm{m}^{2}$, PC membranes with suspensions requiring in total an area of $15 \times 15 \mu \mathrm{m}^{2}$, and additionally the electronics. As discussed in Sections 12.3 and 12.4 the filters are considered with overlapping tuning ranges, just to ensure reliable operation. Similar to previous cases, here the neighboring MEMS filters share their supporting posts in between the filters. Considering the additional space for contacts of sensing and actuation diodes, the estimate yields $0.33 \mathrm{~mm}^{2}$ for the total chip size. The papers do not provide enough information concerning space for signal processing, electronics and contacts. However, extrapolating from what is visible in Figure 1c in [77], we assume that there might be a miniaturization potential of a factor of 6 , which is considered in our estimation.

\subsection{AWG Covering $500 \mathrm{~nm}$ in the NIR Spectral Range}

During his time at the company NTT, Japan, Y. Yoshikuni et al. [24] performed pioneering work on arrayed waveguide gratings (AWGs). His team demonstrated 64 channels with 
$50 \mathrm{GHz}$ frequency spacing for fiberoptic long-haul telecommunication at $1.55 \mu \mathrm{m}$. The corresponding sample was already downscaled and had lateral dimensions of $7 \times 3.6 \mathrm{~mm}^{2}$ [22-24]. At the time of publication, they also implemented the most complex InP photonic device integration in the world, including semiconductor optical amplifiers, photodiode arrays and several AWGs. On the basis of these early and also recent publications, a space requirement of $50 \mathrm{~mm}^{2}$ including 125 channels was estimated for the NIR spectral range.

\subsection{Plasmonic MEMS Cantilever Covering $500 \mathrm{~nm}$ in the NIR Spectral Range}

The device presented by Oshita et al. already shows a very broad tuning range of $300 \mathrm{~nm}$ in the NIR spectral range [68]. Increasing this parameter could be accomplished by a larger stroke of the deflection angle, but this will be limited by several constraints. Flatness of the cantilever during actuation is crucial as the coupling condition to a SPP is angle dependent and bending of the grating would lead to increased FWHM. Reducing this cantilever size will decrease the efficiency of light coupling to the grating considerably. There may be some room for improvement based on optimization of the geometric shape of the device. In the end, using a second device with different spectral tuning range to reach $500 \mathrm{~nm}$ spectral span seems to be the more promising option. In such implementation, two identical MEMS cantilevers would be fabricated, but different grating structures and possible different metals would lead to SPP resonance excitation in different spectral ranges for the same deflection angle. The required space of such a sensor system would be around $20 \mathrm{~mm}^{2}$ if no reduction in size of the single devices is considered.

\subsection{Locally Heated Chirped FBG to Cover a Spectral Span of $500 \mathrm{~nm}$ in the NIR Spectral Range}

Tunable filters based on a chirped FBG as reported by Li et al. [78] work only for a limited spectral span of 15-20 nm. Therefore, tuning over a range of $500 \mathrm{~nm}$ would require at least 30 individual chirped fibers. Since the outer diameter of a conventional single-mode fiber is only $125 \mu \mathrm{m}$, even an array of 30 fibers, positioned for example in a template of etched channels on a silicon substrate, would only require $5 \mathrm{~mm}$ in width. The length of the chirped FBG is given by the required number of periods and is in this case in the range of $50 \mathrm{~mm}$. The presented sensor is not yet optimized for miniaturization since the thermal actuation is based on a heating wire attached to a very bulky linear positioning stage. A possible approach that will not lead to any further increase of the device size would be to integrate an array of heater elements into the silicon wafer template. This would finally lead to a space requirement of $250 \mathrm{~mm}^{2}$ for the sensor alone, excluding other elements such as a photodiode, any further optical fibers for wave transport, coupling elements or control of the heater array.

\section{Where Are the Minimum Structure Size Limits in 3D Nanoimprint Lithography?}

The potential of 2D nanoimprint lithography for replication of extremely small structures was demonstrated by patterns with $5 \mathrm{~nm}$ lateral half-pitch [117], and most recently, even a reduction to $2 \mathrm{~nm}$ was reported [118]. The $5 \mathrm{~nm}$ dimension were imprinted with a III/V semiconductor stamp. It was grown by molecular beam epitaxy, subsequently cleaved and selectively etched on the cleaved side. Finally, the cleaved side represented the nanoimprint stamp [117]. An even more exotic stamp is a substrate with carbon nanotubes on top. $2 \mathrm{~nm}$ in lateral direction were demonstrated by imprinting with these carbon nanotubes [118]. In addition to experimental investigations, theoretical studies have also been performed by modeling the stamp-resist interface. The most relevant forces between the stamp surface and the nanoimprint resist surface, considering the individual atoms of both surfaces, were studied in sub-nanoscale using theoretical model calculations $[119,120]$. The result shown that the stamp could not be released from the cured and hardened imprint resist if lateral structure dimensions are below $0.5 \mathrm{~nm}$. This represents a theoretical limit for minimum lateral size dimensions for nanoimprint lithography. In general, the smaller the molecules used in the nanoimprint resist, the smaller the minimum structure dimensions 
which are obtainable via nanoimprint lithography. Typically, a monomer has a size of 1-2 nm, i.e., even for short-chained polymers, only a small space is remaining in the structure.

Many different template materials are possible, such as glass, Si or III/V semiconductors. Imprints can be directly performed using the template, as what was done in our experiments using GaAs templates. Alternatively, the master template (mother-structure) has also been replicated into polydimethylsiloxane (PDMS) serving as a daughter-structure which is used for the subsequent imprinting in mr-UVcur06. This resist is cured with UV light.

In the following discussion, an intuitive visualization is made in Figure 28 to illustrate the vertical mesa height differences in the sub-nm range in relation to the scale of resist molecules. For the purpose of simplicity, the influence of surface roughness is not included.

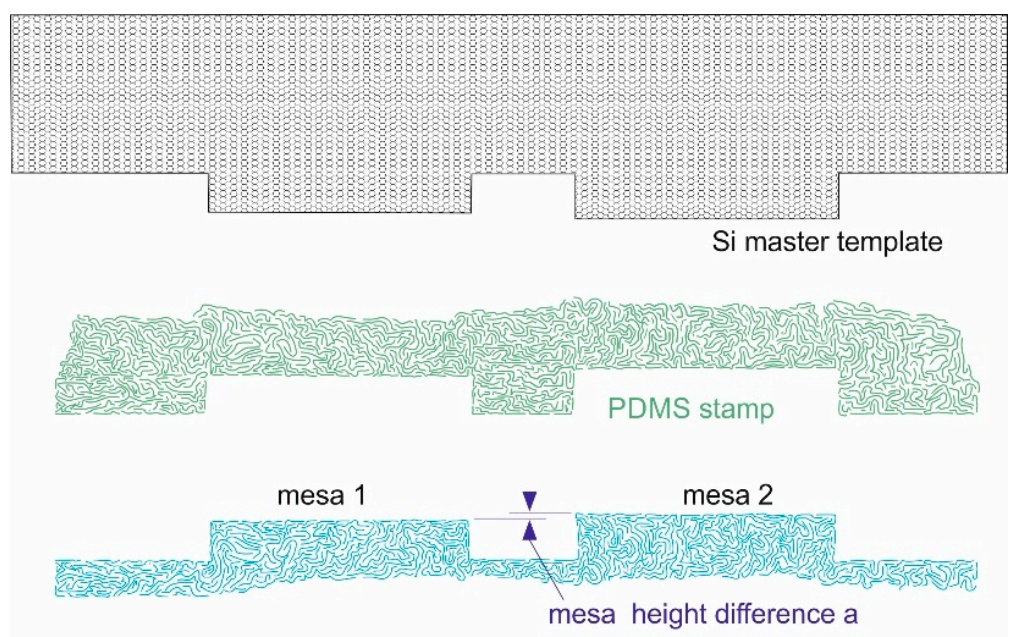

imprinted and hardened cavity structure

Figure 28. Cross sections of schematic surface profiles. The crystalline structure of the Si master template is visualized by the periodic honey-comb structure (top). The master template is replicated in PDMS and rotated by $180^{\circ}$, as shown in green (center). The schematic includes a possible arrangement of organic molecules in the PDMS. Subsequently, 3D nanoimprints were performed using this PDMS stamp. After imprinting the resist, hardening the resist and releasing the stamp the blue schematic surface is resulting (bottom). The figures do not have the same scale in vertical and lateral direction: differences exist in vertical mesa heights between $a=0.2 \mathrm{~nm}$ and $1 \mathrm{~nm}$, lateral mesa widths between $6 \mu \mathrm{m}$ and $40 \mu \mathrm{m}$, and vertical mesa heights between $10 \mathrm{~nm}$ and $300 \mathrm{~nm}$.

Our smallest height difference which was measured with an interference microscopy was $a=0.2 \mathrm{~nm}[33,34]$. On a first glance, this result might be in contradiction to the smallest lateral structure sizes of $0.5 \mathrm{~nm}$ which were predicted in the abovementioned theoretical model calculations $[119,120]$. However, there is no contradiction since the lateral dimensions of our mesa are in the range of $>6 \mu \mathrm{m}$ (see Section 12) or even $40 \mu \mathrm{m}$ in our proof-of-principle (Sections 2-4). The chains of the nanoimprint resist molecules will spread in lateral directions. This means that a very high vertical accuracy can be obtained if comparably large lateral dimensions can be tolerated or are even required as in our case. Furthermore, very high vertical resolution can be obtained if larger residual layers are acceptable or reservoir for excess resist are included in the design. Even if our technology which enables zero-residual layers is used [92], these reservoir technologies and volume equalizing technologies [86] have to be applied.

Measuring $a=0.2 \mathrm{~nm}[33,34]$ using an interference microscopy is executed by averaging over an area containing many polymer chains (diffraction-limited focal diameter). Please note that the exact same approach is applied in the optical sensor, therefore, this is a relevant value for the height differences.

A schematic FP array structures with two different mesa heights is displayed in Figure 25 to envisage vertical resolution limits in 3D nanoimprint lithography. The polymer 
chains of the nanoimprint resist reveal different lengths and shape, and they have a lateral thickness of approximatively $0.2 \mathrm{~nm}$. In lateral directions, the molecules can easily migrate and spread during the filling of the stamp (mold) with imprint resist. Due to these microfluidic aspects, our 3D nanoimprints could reveal vertical mesa height differences down to $0.2 \mathrm{~nm}$ since the lateral dimension is large enough to allow material migration and spreading. In summary, 2D nanoimprint technology uses constant vertical step heights and the smallest structure sizes in lateral directions were $2-5 \mathrm{~nm}$ in the experiments $[117,118]$ and $0.5 \mathrm{~nm}$ in theoretical model calculations [119,120], as already mentioned. In our 3D nanoimprint experiments, $0.2 \mathrm{~nm}$ in mesa height difference was obtained in structures with rather large lateral sizes in the range of several $\mu \mathrm{m}$, due to viable allow material migration and spreading. At the moment, it is hard to estimate the limits for 3D nanoimprint if ultra-small variations are involved in vertical and lateral dimension simultaneously, but it could be in the sub-nm range in all combined three directions.

\section{Can Nanoimprint Be Applied to Fabricate the Seven Sensor Types Compared Here?}

Today, a variety of molding technologies exist, such hot embossing, nanoimprint, injection molding and LIGA. The acronym LIGA consist of the German words Lithographie, Galvanik, Abformung, which means (X-ray) lithography, electroplating, molding [121] and is used worldwide. In nanoimprint technology, structures are replicated using a stamp with structure sizes between a few $\mathrm{nm}$ up to several $\mu \mathrm{m}$. In contrast, LIGA technology is replicating structures from a stamp generating structures between a few $\mu \mathrm{m}$ to several $\mathrm{mm}$ [121]. Even larger objects are replicated by injection molding ranging in size between several $\mu \mathrm{m}$ to the range of a few meters.

Using molding technologies, miniaturized grating spectrometers [72] can be generated to define curved mirrors, grating, cavities to insert detector arrays, guide elements and housing parts in a single step. To lower fabrication cost, molding is always a very good strategy. Micro-grating spectrometers were also replicated applying LIGA processes [122]. Using InGaInAs, waveguide structures were lithographically treated and etched to define transmission grating, curved mirrors and trenches for optical fibers [123].

In this last section, the question is raised whether nanoimprint can be applied in the fabrication of AWG sensors, miniaturized spectrometers, plasmonic MEMS sensors, static FP filter array sensors, MEMS tunable FP array, tunable chirped fiber Bragg gratings, and MEMS tunable PC arrays, all of which are shown in Figure 1. Several advantages of nanoimprint concerning cost, time, and effort were already presented and confirmed by results presented in the previous sections. The lithography included in manufacturing can be replaced by nanoimprint lithography in five out of the seven sensor types. However, in cannot be used in the fabrication of the classical grating spectrometers and the chirped fiber Bragg gratings. Moreover, nanoimprint might not be ideal for the precise positioning of the QDs within the PC structure [77]. We expect that the positioning of the QD would be arduous and might require, e.g., electron or ion beam lithography. Thus, nanoimprint lithography might not reveal clear advantages in manufacturing of MEMS tunable PC structures. However, it is highly desirable to use nanoimprint lithography for the fabrication of AWGs since it has clear advantages in replacing optical lithography in large scale production. Furthermore, nanoimprint is very desirable, but not absolutely necessary, in manufacturing MEMS tunable FP filter arrays. Most essential is it for static FP filter arrays. Using nanoimprint technology, the whole cavity structure including millions of FP filters (pixels) can be imprinted in a single step. Furthermore, the stamp/mold is reusable for many times. Theoretically, there is no limit in the number of FP filters (pixels) which can be defined within a single imprint step that generates all the different 3D cavities altogether [33-37]. Alternative methodologies have to use many more fabrication steps [28-31], which limits the number of pixels which can be manufactured in a realistic way. Among all the considered sensor types, the static FP filters make the most of the advantages of 3D nanoimprint lithography. Many FP filter arrays can be imprinted in a batch process at the same time, potentially resulting in a significant cost reduction. For 
repetitive processes in large scale (mass production), nanoimprint lithography reveals its characteristic superiority in saving time, cost and effort.

\section{Conclusions}

Limits of wide wavelength tuning, FWHM and stopband widths are investigated for the InP multiple air-gap system. Wavelength tuning efficiency for said sensor system is found to be the closest to 1 , and additionally with much smaller size compared to other systems and different sensor methodologies.

In our comparison, the tunable chirped fiber Bragg grating reveals by far the smallest FWHM of $0.007 \mathrm{~nm}$ at $1.5 \mu \mathrm{m}$. However, the space requirement is the largest in this case. The next lowest FWHM of typically $0.1 \mathrm{~nm}$ at $\lambda=1.3 \mu \mathrm{m}$ are measured in MEMS tunable PC filters. Potentially, the space requirement is also very small. However, in order to obtain larger spectral spans of e.g., $400 \mathrm{~nm}$, the combination of several neighboring spectral tuning ranges is required. For that purpose, the QD have to be adjusted and varied in all the PC crystal in a defined way. This might be quite challenging concerning the spectral adjustment and defined variation from array to array. On the other hand, AWGs also provide small linewidths, and the arrangement of several arrays next to each other is very easy. However, the fabrication of AWGs for the VIS spectral range is still an enormous challenge.

The typical linewidths measured for FP filter arrays are higher than the typical values of PC or AWG sensors. Although $0.1 \mathrm{~nm}$ linewidth could be achieved at $\lambda=1.5 \mu \mathrm{m}$ in an InP/multiple air-gap FP filter in a single case, it was not reproducible in tunable filters or in static FP filter arrays. Using the InP multiple airgap MEMS technology, very small linewidths are extremely challenging in the tunable technology, but on the other hand, it is very attractive in terms of scalability. Since various static FP filter arrays can be manufactured next to each other within a single 3D nanoimprint step, this technology enables lowest tentative price per spectral range for visible spectral ranges.

A chance to achieve small linewidths in MEMS tunable filters lies in the application of stable cavities. A small linewidth of $<0.15 \mathrm{~nm}$ was reported over the whole tuning range, although the tuning range is limited and only thermal tuning was applied [124].

The current version of the plasmonic MEMS sensor evaluates charge carriers induced by the SPP resonance into a diode structure and transforming this angle dependent current into the spectral information. At the moment this is limited to a rather high FWHM value of $10 \mathrm{~nm}$ or more. On the other hand, this concept is very promising due to its wide tuning range.

Thermally tuned chirped FBG shows narrow band filter lines with FWHM of $0.007 \mathrm{~nm}$ and can potentially be implemented in any working spectral range of an optical fiber. The small tuning rang of only $16.5 \mathrm{~nm}$ requires, however, an array of many individual chirped FBG to cover broader spectral spans.

The classical grating spectrometer is definitively the best in our comparison in terms of the efficiency in making most out of available light. However, the grating spectrometer suffers considerably from strongly reduced spectral resolution when downscaling the devices, whereas such limitations are not relevant for all the other sensor types compared in our review. In all these cases, the resolution is very high and independent from miniaturization. The AWG uses available light much more efficiently than the static and tunable FP filter arrays and the tunable PC filter array. The latter three own rather low efficiencies, but the efficiencies can be boosted by spectral preselection as shown in this review.

Fourier spectroscopy in the infrared spectral range is using the amount of light much more efficiently than grating spectrometers (Multiplex and Jacquinot advantages) in large set-ups. The disadvantage is that the miniaturization achieved up to now is by far less than that obtained for PC and FP-based sensors.

Nanoimprint can be applied to all the compared sensors, except the chirped fiber Bragg grating and the classical grating spectrometer. Transmission gratings could be fabricated by nanoimprint lithography. Notwithstanding, nanoimprint technology can only reveal its full potential in manufacturing static FP filter arrays. Here, 192 different filter lines were demonstrated using a single 3D nanoimprint step to define accurate and 
diverse 3D cavity layers. In a proof-of-concept, 192 spectrally different filter lines were successfully demonstrated, which is far better than the three different broad filter lines used in modern digital cameras. There are no limits on the principle to considerably increase these values in static FP filter arrays. At the same time, nanoimprint substantially reduces fabrication time, cost and effort.

\section{Patents}

For FP filter arrays, PC sensors and plasmonic sensors, the obvious disadvantage concerning less efficiency in using available light (in comparison to grating spectrometers) can be partly compensated by spectral preselection: R. Brunner, H. Hillmer and A. Gatto: Spektralsensor zur spektralen Analyse einfallenden Lichts, German Patent 2016, DE 102014 108138 B4. Recently, this concept has been proven, experimentally [11,89]. See Section 6.

Author Contributions: Conceptualization, H.H., T.K. and R.B.; Methodology, H.H., T.K., R.B., C.W., A.K., M.K. and M.S.Q.I.; Validation, A.K., C.W., A.I., T.K., M.S.Q.I. and H.H.; Formal Analysis, M.S.Q.I., A.K., M.K., H.H. and T.K.; Investigation, A.K., A.I., M.S.Q.I. and C.W.; Writing-Original Draft Preparation, H.H., T.K. and A.K.; Writing-Review and Editing, all authors; Visualization, H.H., T.K., A.I. and A.K.; Funding Acquisition, H.H. and R.B. All authors have read and agreed to the published version of the manuscript.

Funding: Financial funding by DFG, BMBF, HA and EU is gratefully acknowledged.

Institutional Review Board Statement: Not applicable.

Informed Consent Statement: Not applicable.

Data Availability Statement: No new data were created or analyzed in this study. Data sharing is not applicable to this article.

Acknowledgments: The authors thank S. Irmer, J. Daleiden, C. Prott, F. Römer, A. Hasse, Y. Shen, R. Zamorra, VR. Kolli, M. Strassner, D. T. Nguyen, N. Ahmed, N. Dharmarasu, T. Nurjahan, A. Friedrichsen, I. Kommallein, J. Krumpholz, D. Gutermuth, H. H. Mai, Waleed Al-Esayi, B. Laue, E. Förster, M. Bartels, M. Ababtain, I. Memon, A. Ullah, P. Lehmann, S. Hansmann, M. Verschuuren, M. Hornung and V. Daneker for stimulating discussions, technological processing and technical support.

Conflicts of Interest: The authors declare no conflict of interest. The funding agencies had no role in the design, execution, interpretation, or writing of the study.

$\begin{array}{ll}\text { Abbreviations } \\ \text { 1D } & \text { one-dimensional } \\ \text { 2D } & \text { two-dimensional } \\ \text { 3D } & \text { three-dimensional } \\ \alpha_{\text {InP }} & \text { absorption coefficient of InP } \\ \alpha_{S i 3 N 4} & \text { absorption coefficient of silicon nitride } \\ \alpha_{S i O 2} & \text { absorption coefficient of silicon dioxide } \\ \mathrm{AWG} & \text { arrayed waveguide grating } \\ \mathrm{CCD} & \text { charge coupled device } \\ \mathrm{CMOS} & \text { complementary metal oxide semiconductor } \\ \Delta \lambda & \text { wavelength tuning range, wavelength interval, wavelength difference } \\ \Delta \lambda_{\mathrm{i}} & \text { wavelength interval, wavelength spacing } \\ \Delta \lambda / \lambda & \text { optical resolution of an optical sensor or spectrometer } \\ \Delta l & \text { interface fluctuations } \\ \Delta L & \text { displacement of DBR mirrors, cavity tuning } \\ \Delta \mathrm{L}_{1}, \Delta \mathrm{L}_{2} & \text { cavity lengths fluctuations } \\ \Delta \lambda / \Delta L & \text { tuning efficiency } \\ \Delta n & \text { refractive index difference } \\ \mathrm{DBR} & \text { distributed Bragg reflector } \\ \mathrm{DWDM} & \text { dense wavelength division multiplex } \\ \mathrm{FBG} & \text { fiber Bragg grating }\end{array}$




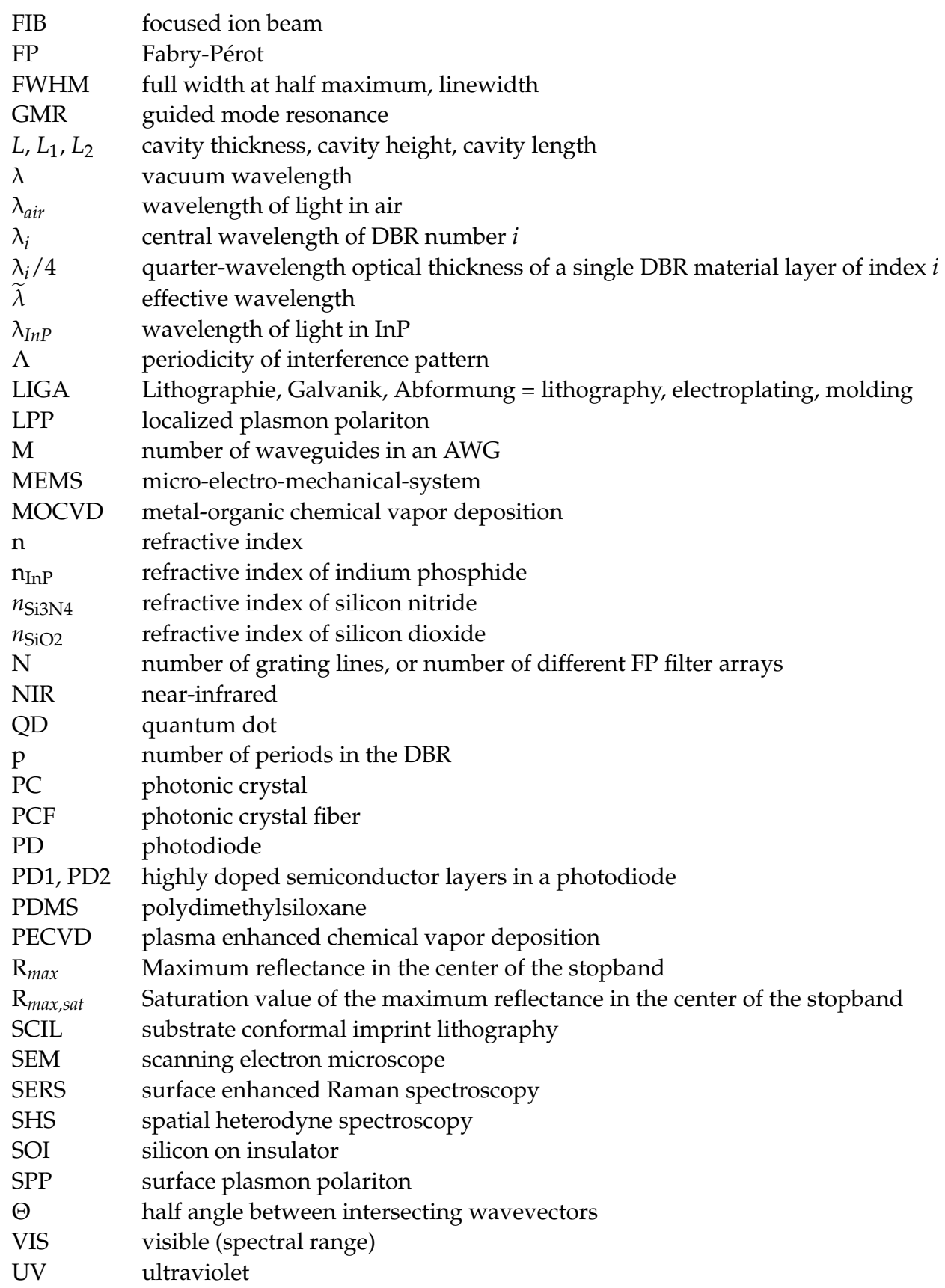

\section{References}

1. Lindon, J.C.; Tranter, G.E.; Koppenaal, D. Encyclopedia of Spectroscopy and Spectrometry, 3rd ed.; Academic Press: Cambridge, MA, USA, 2016; ISBN 978-0-12-803224-4.

2. Haken, H.; Wolf, H.C. Modern Methods of Optical Spectroscopy. In The Physics of Atoms and Quanta; Springer: Berlin, Heidelberg, Germany, 1996. [CrossRef]

3. Baeten, V.; Dardenne, P. Spectroscopy: Developments in Instrumentation and Analysis. Grasas Aceites 2002, 53, 45-63. [CrossRef]

4. Tkachenko, N.V. Optical Spectroscopy: Methods and Instrumentations, 1st ed.; Elsevier: Amsterdam, The Netherlands, 2006; ISBN 978-0-444-52126-2.

5. Dakin, J.P.; Chambers, P. Review of Methods of Optical GAS detection by Direct Optical Spectroscopy, with Emphasis on Correlation Spectroscopy. In Optical Chemical Sensors; NATO Science Series; II: Mathematics, Physics and Chemistry; Baldini, F., Chester, A., Homola, J., Martellucci, S., Eds.; Springer: Dordrecht, The Netherlands, 2006; Volume 224, pp. 457-477. ISBN 978-14020-4609-4.

6. Hodgkinson, J.; Tatam, R.P. Optical gas sensing: A review. Meas. Sci. Technol. 2013, 24, 012004. [CrossRef]

7. Rolinger, L.; Rüdt, M.; Hubbuch, J. A critical review of recent trends, and a future perspective of optical spectroscopy as PAT in biopharmaceutical downstream processing. Anal. Bioanal. Chem. 2020, 412, 2047-2064. [CrossRef] 
8. Neumann, W. Fundamentals of Dispersive Optical Spectroscopy Systems; Society of Photo-Optical Instrumentation Engineers (SPIE): Bellingham, WA, USA, 2013; PM242; ISBN 978-081-949-824-3.

9. Appenzeller, I. Optical-Range Grating and Prism Spectrometers. In Introduction to Astronomical Spectroscopy (Cambridge Observing Handbooks for Research Astronomers); Cambridge University Press: Cambridge, UK, 2012; pp. 81-126. [CrossRef]

10. Thorne, A.P. Dispersion and resolving power: Prism spectrographs. In Spectrophysics; Springer: Dordrecht, The Netherlands, 1988. [CrossRef]

11. Hillmer, H.; Woidt, C.; Istock, A.; Kobylinskiy, A.; Nguyen, D.T.; Ahmed, N.; Brunner, R.; Kusserow, T. Role of Nanoimprint Lithography for Strongly Miniaturized Optical Spectrometers. Nanomaterials 2021, 11, 164. [CrossRef]

12. Kenda, A.; Frank, A.; Kraft, M.; Tortschanoff, A.; Sandner, T.; Schenk, H.; Scherf, W. Compact High-Speed Spectrometers Based on MEMS Devices with Large Amplitude In-Plane Actuators. Procedia Chem. 2009, 1, 556-559. [CrossRef]

13. Tormen, M.; Lockhart, R.; Niedermann, P.; Overstolz, T.; Hoogerwerf, A.; Mayor, J.-M.; Pierer, J.; Bosshard, C.; Ischer, R.; Voirin, G.; et al. MEMS tunable grating micro-spectrometer. In Proceedings of the International Conference on Space OpticsICSO, Toulouse, France, 14-17 October 2008; Society of Photo-Optical Instrumentation Engineers (SPIE): Bellingham, WA, USA, 2008; p. 1056607. [CrossRef]

14. Huang, J.; Wen, Q.; Nie, Q.; Chang, F.; Zhou, Y.; Wen, Z. Miniaturized NIR Spectrometer Based on Novel MOEMS Scanning Tilted Grating. Micromachines 2018, 9, 478. [CrossRef]

15. Truxal, S.T.; Kurabayashi, K.; Tung, J.-C. Design of a MEMS Tunable Polymer Grating for Single Detector Spectroscopy. Int. J. Optomechatronics 2008, 2, 75-87. [CrossRef]

16. Harmon, K. Interferometers: Fundamentals, Methods and Applications (Physics Research and Technology); Nova Science Publishers: Hauppauge, NY, USA, 2015; ISBN 978-1-63483-692-0.

17. Hariharan, P. Basics of Interferometry; Academic Press: Boston, MA, USA, 1992; ISBN 978-012-325-218-0.

18. Andersson, P.O.; Edwall, G.; Persson, A.; Thylén, L. Fiber Optic Mach-Zehnder Interferometer Based on Lithium Niobate Components. In Integrated Optics; Springer Series in Optical, Sciences; Nolting, H.P.J., Ulrich, R., Eds.; Springer: Berlin/Heidelberg, Germany, 1985; Volume 48, pp. 26-28. ISBN 978-3-540-39452-5.

19. Zetie., K.P.; Adams, S.F.; Tocknell, R.M. How does a Mach-Zehnder interferometer work? Phys. Educ. 2000, 35, 46-48. [CrossRef]

20. Russel, J.; Cohn, R. Mach-Zehnder Interferometer; Book on Demand: Norderstedt, Germany, 2013; ISBN 978-551-267-842-8.

21. Smit, M.K.; van Dam, C. PHASAR-Based WDM-Devices: Principles, Design and Applications. IEEE J. Sel. Top. Quantum Electron. 1996, 2, 236-250. [CrossRef]

22. Kohtoku, M.; Sanjoh, H.; Oku, S.; Kadota, Y.; Yoshikuni, Y.; Shibata, Y. InP-based 64-channel arrayed waveguide grating with $50 \mathrm{GHz}$ channel spacing and up to $-20 \mathrm{~dB}$ crosstalk. Electron. Lett. 1997, 33, 1786-1787. [CrossRef]

23. Kohtoku, M.; Sanjoh, H.; Oku, S.; Kadota, Y.; Yoshikuni, Y. Polarization Independent Semiconductor Arrayed Waveguide Gratings Using a Deep-Ridge Waveguide Structure. IEICE Trans. Electron. 1998, E81-C, 1195-1204.

24. Yoshikuni, Y. Semiconductor Arrayed Waveguide Gratings for Photonic Integrated Devices. IEEE J. Sel. Top. Quantum Electron. 2002, 8, 1102-1114. [CrossRef]

25. Cvetojevic, N.; Jovanovic, N.; Bland-Hawthorn, J.; Haynes, R.; Lawrence, J. Miniature Spectrographs: Characterization of Arrayed Waveguide Gratings for Astronomy. Proc. SPIE Int. Soc. Opt. Eng. 2010, 77394H. [CrossRef]

26. Seyringer, D.; Sagmeister, M.; Maese-Novo, A.; Eggeling, M.; Rank, R.; Muellner, P.; Hainberger, R.; Drexler, W.; Vlaskovic, M.; Zimmermann, H.; et al. Technological verification of size-optimized 160-channel silicon nitride-based AWG-spectrometer for medical applications. Appl. Phys. B 2019, 125, 88. [CrossRef]

27. Muneeb, M.; Ruocco, A.; Malik, A.; Pathak, S.; Ryckeboer, E.; Sanchez, D.; Cerutti, L.; Rodriguez, J.B.; Tournié, E.; Bogaerts, W.; et al. Silicon-on-insulator shortwave infrared wavelength meter with integrated photodiodes for on-chip laser monitoring. Opt. Express 2014, 22, 27300-27308. [CrossRef]

28. Correia, J.H.; Bartek, M.; Wolffenbuttel, R.F. High-selectivity single-chip spectrometer in silicon for operation in visible part of the spectrum. IEEE Trans. Electron. Devices 2000, 47, 191-197. [CrossRef]

29. Correia, J.H.; de Graaf, G.; Kong, S.H.; Bartek, M.; Wolffenbuttel, R.F. Single-chip CMOS optical micro-spectrometer. Sens. Actuators 2000, 82, 191-197. [CrossRef]

30. Wolffenbuttel, R.F. MEMS-based optical mini- and microspectrometers for the visible and infrared spectral range. J. Micromech. Microeng. 2005, 15, 145-152. [CrossRef]

31. Wang, S.W.; Li, M.; Xia, C.S.; Wang, H.Q.; Chen, X.S.; Lu, W. 128 channels of integrated filter array rapidly fabricated by using the combinatorial deposition technique. Appl. Phys. B 2007, 88, 281-284. [CrossRef]

32. Hillmer, H. Optisches Filter und Verfahren zu Seiner Herstellung (Optical Filter and Its Fabrication Technology). Patent DE 10,2006,039,071, 19 April 2012.

33. Albrecht, A.; Wang, X.; Mai, H.H.; Schotzko, T.; Memon, I.; Hornung, M.; Bartels, M.; Hillmer, H. High vertical resolution 3D Nano-Imprint Technology and its application in optical nanosensors. Nonlinear Opt. Quantum Opt. 2012, 43, $339-353$.

34. Wang, X.; Albrecht, A.; Mai, H.H.; Woidt, C.; Meinl, T.; Hornung, M.; Bartels, M.; Hillmer, H. High resolution 3D nanoimprint technology: Template fabrication, application in Fabry-Pérot filter-array-based optical nanospectrometers. Microelectron. Eng. 2013, 110, 44-51. [CrossRef] 
35. Mai, H.H.; Albrecht, A.; Woidt, C.; Wang, X.; Daneker, V.; Setyawati, O.; Woit, T.; Schultz, K.; Bartels, M.; Hillmer, H. 3D nanoimprinted Fabry-Pérot filter arrays and methodologies for optical characterization. Appl. Phys. B Lasers Opt. 2012, 107, 755-764. [CrossRef]

36. Nguyen, D.T.; Ababtain, M.; Memon, I.; Ullah, A.; Istock, A.; Woidt, C.; Xie, W.; Lehmann, P.; Hillmer, H. 3D nanoimprint for NIR Fabry-Pérot filter arrays: Fabrication, characterization and comparison of different cavity designs. Appl. Nanosci. 2016, 6, 1127-1135. [CrossRef]

37. Shen, Y.; Istock, A.; Zaman, A.; Woidt, C.; Hillmer, H. Fabrication and characterization of multi-stopband Fabry-Pérot filter array for nanospectrometers in the VIS range using SCIL nanoimprint technology. Appl. Nanosci. 2018, 8, 1415-1425. [CrossRef]

38. Larson, M.C.; Harris, J.S. Broadly-tunable resonant-cavity light-emitting diode. IEEE Photonics Technol. Lett. 1995, 7, 1267-1269. [CrossRef]

39. Larson, M.C.; Pezeshki, B.; Harris, J.S. Vertical Coupled-Cavity Microinterferometer on GaAs with Deformable-Membrane Top Mirror. IEEE Photonics Technol. Lett. 1995, 7, 382-384. [CrossRef]

40. Vail, E.C.; Wu, M.S.; Li, G.S.; Eng, L.; Chang-Hasnain, C.J. GaAs micromachined widely tunable Fabry-Perot filters. Electron. Lett. 1995, 31, 228-229. [CrossRef]

41. Wu, M.S.; Vail, E.C.; Li, G.S.; Yuen, W.; Chang-Hasnain, C.J. Tunable micromachined vertical cavity surface emitting laser. Electron. Lett. 1995, 31, 1671-1672. [CrossRef]

42. Spisser, A.; Ledantec, R.; Seassal, C.; Leclercq, J.L.; Benyattou, T.; Rondi, D.; Blondeau, R.; Guillot, G.; Viktorovitch, P. Highly selective and widely tunable 1.55-mInP/Air-Gap micromachined Fabry-Perot filter for optical communications. IEEE Photonics Technol. Lett. 1998, 10, 1259-1261. [CrossRef]

43. Tayebati, P.; Wang, P.; Azimi, M.; Maflah, L.; Vakhshoori, D. Microelectromechanical tunable filter with stable half symmetric cavity. Electron. Lett. 1998, 34, 1967-1968. [CrossRef]

44. Peerlings, J.; Dehe, A.; Vogt, A.; Tilsch, M.; Hebeler, C.; Langenhan, F.; Meissner, P.; Hartnagel, H.L. Long resonator micromachined tunable GaAs-AlAs Fabry-Perot filter. IEEE Photonics Technol. Lett. 1997, 9, 1235-1237. [CrossRef]

45. Streubel, K.; Rapp, S.; Andre, J.; Chitica, N. $1.26 \mu m$ vertical cavity laser with two InP/air-gap reflectors. Electron. Lett. 1996, 32, 1369-1370. [CrossRef]

46. Le Dantec, R.; Benyattou, T.; Guillot, G.; Spisser, G.; Seassal, C.; Leclercq, J.L.; Victorovitch, P.; Blondeau, R. Tunable microcavity based on InP-Air Bragg mirrors. IEEE J. Sel. Top. Quantum Electron. 1999, 5, 111-114. [CrossRef]

47. Hillmer, H.; Daleiden, J.; Prott, C.; Römer, F.; Irmer, S.; Rangelov, V.; Tarraf, A.; Schüler, S.; Strassner, M. Potential for micromachined actuation of ultra-wide continuously tunable optoelectronic devices. Appl. Phys. B 2002, 75, 3-13. [CrossRef]

48. Daleiden, J.; Chitica, N.; Strassner, M.; Spisser, A.; Leclercq, J.L.; Victorovitch, P.; Rondi, D.; Goutain, E.; Peerlings, J.; Pfeiffer, J.; et al. Tunable InP/air gap Fabry Perot filter for wavelength division multiplex fiber optical transmission. In Proceedings of the 11th International Conference on Indium Phosphide and Related Materials (IPRM'99) (Cat. No.99CH36362), Davos, Switzerland, 16-20 May 1999; pp. 285-287. [CrossRef]

49. Strassner, M.; Daleiden, J.; Chitica, N.; Keiper, D.; Stålnacke, B.; Greek, D.; Hjort, K. III-V semiconductor material for tunable Fabry-Perrot filters for coarse and dense WDM systems. Sens. Actuators A Phys. 2000, 85, 249-255. [CrossRef]

50. Chitica, N.; Strassner, M. Room-temperature operation of photopumped monolithic InP vertical cavity laser with two air-gap Bragg reflectors. Appl. Phys. Lett. 2001, 78, 3935-3937. [CrossRef]

51. Irmer, S.; Daleiden, J.; Rangelov, V.; Prott, C.; Römer, F.; Strassner, M.; Tarraf, A.; Hillmer, H. Ultralow biased widely continuously tunable fabry-Perot filter. IEEE Photonics Technol. Lett. 2003, 15, 434-436. [CrossRef]

52. Daleiden, J.; Hillmer, H. Multiple air-gap filters and constricted mesa lasers-Material processing meets the front of optical device technology. Appl. Phys. B 2003, 76, 821-832. [CrossRef]

53. Hillmer, H.; Daleiden, J.; Irmer, S.; Römer, F.; Prott, C.; Tarraf, A.; Strassner, M.; Ataro, E.; Scholz, T. Potential of micromachined photonics: Miniaturization, scaling and applications in continuously tunable vertical air-cavity filters. In Laser Diodes, Optoelectronic Devices, and Heterogenous Integration; International Society for Optics and Photonics: Bellingham, WA, USA, 2003; Volume 4947, pp. 197-211. [CrossRef]

54. Prott, C.; Römer, F.; Ataro, E.O.; Daleiden, J.; Irmer, S.; Tarraf, A.; Hillmer, H. Modeling of ultrawidely tunable vertical cavity air-gap filters and VCSELs. IEEE J. Sel. Top. Quantum Electron. 2003, 9, 918-928. [CrossRef]

55. Römer, F.; Prott, C.; Irmer, S.; Daleiden, J.; Tarraf, A.; Hillmer, H.; Strassner, M. Tuning efficiency and linewidth of electrostatically actuated multiple air-gap filters. Appl. Phys. Lett. 2003, 82, 176-178. [CrossRef]

56. Hasse, A.; Irmer, S.; Daleiden, J.; Dharmarasu, N.; Hansmann, S.; Hillmer, H. Wide continuous tuning range of 221nm by InP /air-gap vertical-cavity filters. Electron. Lett. 2006, 42, 974-975. [CrossRef]

57. Römer, F. Charakterisierung und Simulation Optischer Eigenschaften von Mikromechanisch Abstimmbaren Filterbauelementen (Characterization and Simulation of Optical Properties of Micromachined Tunable Filter Devices); Kassel University Press: Kassel, Germany, 2006; ISBN 978-3-89958-196-6.

58. Williams, C.; Hong, N.; Julian, M.; Borg, S.; Kim, H.J. Tunable mid-wave infrared Fabry-Perot bandpass filters using phase-change GeSbTe. Opt. Express 2020, 28, 10583-10594. [CrossRef]

59. Magnusson, R.; Wang, S.S. New principle for optical filters. Appl. Phys. Lett. 1992, 61, 1022-1024. [CrossRef] 
60. Kanskar, M.; Paddon, P.; Pacradouni, V.; Morin, R.; Busch, A.; Young, J.F.; Johnson, S.R.; MacKenzie, J.; Tiedje, T. Observation of leaky slab modes in an air-bridged semiconductor waveguide with a two-dimensional photonic lattice. Appl. Phys. Lett. 1997, 70, 1438-1440. [CrossRef]

61. Hessel, O.; Oliner, A.A. A new theory of Wood's anomalies on optical gratings. Appl. Opt. 1965, 4, 1275-1297. [CrossRef]

62. Boutami, S.; Benbakir, B.; Letartre, X.; Leclercq, J.L.; Regreny, P.; Viktorovitch, P. Ultimate vertical Fabry-Perot cavity based on single-layer photonic crystal mirrors. Opt. Express 2007, 15, 12443-12449. [CrossRef]

63. Boutami, S.; Bakir, B.B.; Hattori, H.; Letartre, X.; Leclercq, J.-L.; Rojo-Rome, P.; Garrigues, M.; Seassal, C.; Viktorovitch, P. Broadband and compact 2-D photonic crystal reflectors with controllable polarization dependence. IEEE Photonics Technol. Lett. 2006, 18, 835-837. [CrossRef]

64. Zamora, R.; Benes, M.; Kusserow, T.; Hillmer, H.; Akcakoca, U.; Witzigmann, B. Optical characterization of photonic crystals as polarizing structures for tunable optical MEMS devices. In Proceedings of the 16th International Conference on Optical MEMS and Nanophotonics, Istanbul, Turkey, 8-11 August 2011; pp. 83-84. [CrossRef]

65. Kupec, J.; Akcakoca, U.; Witzigmann, B. Frequency domain analysis of guided resonances and polarization selectivity in photonic crystal membranes. J. Opt. Soc. Am. B 2011, 28, 69-78. [CrossRef]

66. Kilic, O.; Digonnet, M.; Kino, G.; Solgaaard, O. Controlling uncoupled resonances in photonic crystals through breaking the mirror symmetry. Opt. Express 2008, 16, 13090-13103. [CrossRef]

67. Kikuta, H.; Toyota, H.; Yu, W. Optical Elements with Subwavelength Structured Surfaces. Opt. Rev. 2003, 10, 63-73. [CrossRef]

68. Oshita, M.; Takahashi, H.; Ajiki, Y.; Kan, T. Reconfigurable Surface Plasmon Resonance Photodetector with a MEMS Deformable Cantilever. ACS Photonics 2020, 7, 673-679. [CrossRef]

69. Momeni, B.; Hosseini, E.S.; Askari, M.; Soltani, M.; Adibi, A. Integrated photonic crystal spectrometers for sensing applications. Opt. Commun. 2009, 282, 3168-3171. [CrossRef]

70. Gerken, M.; Miller, D.A.B. Multilayer Thin-Film Structures with High Spatial Dispersion. Appl. Opt. 2003, 42, 1330-1345. [CrossRef]

71. Ibsen Photonics A/S. OEM Spectrometers. Available online: https://ibsen.com/products/oem-spectrometers/ (accessed on 17 July 2021).

72. Hamamatsu Photonics, K.K. Mini-Spectrometers. Available online: https://www.hamamatsu.com/eu/en/product/opticalsensors / spectrometers/mini-spectrometer/index.html (accessed on 29 November 2020).

73. Suh, W.; Yanik, M.F.; Solgaard, O.; Fan, S. Displacement-sensitive photonic crystal structures based on guided resonance in photonic crystal slabs. Appl. Phys. Lett. 2003, 82, 1999. [CrossRef]

74. Notomi, M.; Taniyama, H.; Mitsugi, S.; Kuramochi, E. Optomechanical Wavelength and Energy Conversion in High-Q DoubleLayer Cavities of Photonic Crystal Slabs. Phys. Rev. Lett. 2006, 97, 023903-4. [CrossRef]

75. Midolo, L.; van Veldhoven, P.J.; Dündar, M.A.; Nötzel, R.; Fiore, A. Electromechanical wavelength tuning of double-membrane photonic crystal cavities. Appl. Phys. Lett. 2011, 98, 211120. [CrossRef]

76. Liapis, A.C.; Gao, B.; Siddiqui, M.R.; Shi, Z.; Boyd, R.W. On-chip spectroscopy with thermally tuned high-Q photonic crystal cavities. Appl. Phys. Lett. 2016, 108, 021105. [CrossRef]

77. Zobenica, Ž.; van der Heijden, R.W.; Petruzzela, M.; Pagliano, F.; Leijssen, R.; Xia, T.; Midolo, L.; Cotrufo, M.; Cho, Y.J.; van Otten, F.W.; et al. Integrated nano-opto-electro-mechanical sensor for spectrometry and nanometrology. Nat. Commun. 2017, 8, 2216. [CrossRef]

78. Yang, Z.; Albow-Owen, T.; Cai, W.; Hasan, T. Miniaturization of optical spectrometers. Science 2021, 371, 480. [CrossRef] [PubMed]

79. Li, S.; Ngo, N.; Tjin, S.; Shum, P.; Zhang, J. Thermally Tunable Narrow-Bandpass Filter Based on a Linearly Chirped Fiber Bragg Grating. Optics Letters 2004, 29, 29-31. [CrossRef]

80. Thomas Rasmussen (Technical Representative of the Company IBSEN Photonics Denmark). Personal communication, 1 July 2021.

81. Michel, A.; Ruprecht, R.; Harmening, M.; Bacher, W. Abformung von Mikrostrukturen auf prozessierten Wafern (Molding of microstructures on processed wafers). Ber. Des. Kernforsch. Karlsr. 1993, KfK 5171. ISSN 0303-4003. [CrossRef]

82. Chou, S.Y.; Krauss, P.R.; Renstrom, P.J. Nanoimprint lithography. J. Vac. Sci. Technol. B Microelectron. Nanometer Struct. 1996, 14, 4129-4133. [CrossRef]

83. Ji, R.; Hornung, M.; Verschuuren, M.A.; van de Laar, R.; van Eeckelen, J.; Plachetka, U.; Moeller, M.; Moormann, C. UV enhanced substrate conformal imprint lithography (UV-SCIL) technique for photonic crystals patterning in LED manufacturing. Microelectron. Eng. 2010, 87, 963-967. [CrossRef]

84. Verschuuren, M.A.; Megens, M.; Ni, Y.; van Sprang, H.; Polman, A. Large area nanoimprint by substrate conformal imprint lithography (SCIL). Adv. Opt. Technol. 2017, 6, 243-264. [CrossRef]

85. Larouche, S.; Martinu, L. OpenFilters: Open-source software for the design, optimization, and synthesis of optical filters. Appl. Opt. 2008, 47, C219-C230. [CrossRef] [PubMed]

86. Memon, I.; Shen, Y.; Khan, A.; Woidt, C.; Hillmer, H. Highly uniform residual layers for arrays of 3D nanoimprinted cavities in Fabry-Pérot filter array based nanospectrometers. Appl. Nanosci. 2015, 6, 599-606. [CrossRef]

87. Emadi, A.; Wu, H.; de Graaf, G.; Wolffenbuttel, R. Design and implementation of a sub-nm resolution microspectrometer based on a Linear-Variable Optical Filter. Opt. Express 2012, 20, 489. [CrossRef]

88. Brunner, R.; Hillmer, H.; Gatto, A. Spektralsensor zur Spektralen Analyse Einfallenden Lichts. Patent DE 10,2014,108,138 B4, 29 December 2016. 
89. Kobylinskiy, A.; Laue, B.; Förster, E.; Höfer, B.; Shen, Y.; Hillmer, H.; Brunner, R. Substantial increase in detection efficiency for filter array-based spectral sensors. Appl. Opt. 2020, 59, 2443-2451. [CrossRef] [PubMed]

90. Abramson, A.R.; Tien, C.; Majumdar, A. Interface and strain effects on the thermal conductivity of heterostructures: A molecular dynamics study. J. Heat Transf. 2002, 124, 963-970. [CrossRef]

91. Cheng, X.; Guo, L.J. A combined-nanoimprint-and-photolithography patterning technique. Microelectron. Eng. 2004, 71, 277-282. [CrossRef]

92. Kolli, V.R.; Woidt, C.; Hillmer, H. Residual-layer-free 3D nanoimprint using hybrid soft templates. Microelectron. Eng. 2016, 149, 159-165. [CrossRef]

93. Wang, Y. Model Calculations and Implementation of Filters and Hybrid Green VCSELs Based on Optical Thin Film Stacks. Ph.D. Thesis, University of Kassel, Kassel, Germany, 2010.

94. Faraji-Dana, M.; Arbabi, E.; Arbabi, A.; Kamali, S.M.; Kwon, H.; Faraon, A. Compact Folded Metasurface Spectrometer. Nat. Commun. 2018, 9, 4196. [CrossRef] [PubMed]

95. Nitkowski, A.; Chen, L.; Lipson, M. Cavity-Enhanced on-Chip Absorption Spectroscopy Using Microring Resonators. Opt. Express 2008, 16, 11930-11936. [CrossRef]

96. Kyotoku, B.B.C.; Chen, L.; Lipson, M. Sub-Nm Resolution Cavity Enhanced Micro-Spectrometer. Opt. Express 2010, 18, 102-107. [CrossRef]

97. Yang, Z.; Albrow-Owen, T.; Cui, H.; Alexander-Webber, J.; Gu, F.; Wang, X.; Wu, T.-C.; Zhuge, M.; Williams, C.; Wang, P.; et al. Single-Nanowire Spectrometers. Science 2019, 365, 1017-1020. [CrossRef] [PubMed]

98. Tabassum, S.; Kumar, R.; Dong, L. Nanopatterned Optical Fiber Tip for Guided Mode Resonance and Application to Gas Sensing. IEEE Sens. J. 2017, 17, 7262-7272. [CrossRef]

99. Ma, C.; Wang, A. Optical Fiber Tip Acoustic Resonator for Hydrogen Sensing. Opt. Lett. 2010, 35, 2043. [CrossRef]

100. Zhu, T.; Ke, T.; Rao, Y.; Chiang, K.S. Fabry-Perot Optical Fiber Tip Sensor for High Temperature Measurement. Opt. Commun. 2010, 283, 3683-3685. [CrossRef]

101. Ma, J.; Jin, W.; Ho, H.L.; Dai, J.Y. High-Sensitivity Fiber-Tip Pressure Sensor with Graphene Diaphragm. Opt. Lett. 2012, 37, 2493. [CrossRef]

102. Kilic, O.; Solgaard, O.; Digonnet, M.; Kino, G. External Fibre Fabry-Perot Acoustic Sensor Based on a Photonic-Crystal Mirror. Meas. Sci. Technol. 2007, 18, 3049-3054. [CrossRef]

103. Alberts, C.J.; Man, S.D.; Berenschot, J.W.; Gadgil, V.J.; Elwenspoek, M.C.; Iannuzzi, D. Fiber-Top Refractometer. Meas. Sci. Technol. 2009, 20, 034005. [CrossRef]

104. De Maria, L.; Martinelli, M.; Vegetti, G. Fiber-Optic Sensor Based on Surface Plasmon Interrogation. Sens. Actuators B Chem. 1993, 12, 221-223. [CrossRef]

105. Kazuma, E.; Tatsuma, T. Localized Surface Plasmon Resonance Sensors Based on Wavelength-Tunable Spectral Dips. Nanoscale 2014, 6, 2397-2405. [CrossRef]

106. Strobbia, P.; Languirand, E.R.; Cullum, B.M. Recent Advances in Plasmonic Nanostructures for Sensing: A Review. Opt. Eng. 2015, 54, 100902. [CrossRef]

107. Alvarez-Puebla, R.; Cui, B.; Bravo-Vasquez, J.-P.; Veres, T.; Fenniri, H. Nanoimprinted SERS-Active Substrates with Tunable Surface Plasmon Resonances. J. Phys. Chem. C 2007, 111, 6720-6723. [CrossRef]

108. Kostovski, G.; Chinnasamy, U.; Jayawardhana, S.; Stoddart, P.R.; Mitchell, A. Sub-15nm Optical Fiber Nanoimprint Lithography: A Parallel, Self-Aligned and Portable Approach. Adv. Mater. 2010, 23, 531-535. [CrossRef]

109. Bierlich, J.; Kobelke, J.; Brand, D.; Kirsch, K.; Dellith, J.; Bartelt, H. Nanoscopic Tip Sensors Fabricated by Gas Phase Etching of Optical Glass Fibers. Photonic Sens. 2012, 2, 331-339. [CrossRef]

110. Burresi, M.; van Oosten, D.; Kampfrath, T.; Schoenmaker, H.; Heideman, R.; Leinse, A.; Kuipers, L. Probing the Magnetic Field of Light at Optical Frequencies. Science 2009, 326, 550-553. [CrossRef] [PubMed]

111. Iannuzzi, D.; Deladi, S.; de Man, S.; Heeck, K.; Elwenspoek, M.C. Fiber-Top Atomic Force Microscope. Rev. Sci. Instrum. 2006, 77, 106105. [CrossRef]

112. Kostovski, G.; Stoddart, P.R.; Mitchell, A. The Optical Fiber Tip: An Inherently Light-Coupled Microscopic Platform for Microand Nanotechnologies. Adv. Mater. 2014, 26, 3798-3820. [CrossRef]

113. Harlander, J.M. Spatial Heterodyne Spectroscopy: Interferometric Performance at Any Wavelength without Scanning. Ph.D. Thesis, University of Wisconsin, Madison, WI, USA, 1991.

114. Zettner, A.; Gojani, A.B.; Schmid, T.; Gornushkin, I.B. Evaluation of a Spatial Heterodyne Spectrometer for Raman Spectroscopy of Minerals. Minerals 2020, 10, 202. [CrossRef]

115. Kaufmann, M.; Olschewski, F.; Mantel, K.; Solheim, B.; Shepherd, G.; Deiml, M.; Liu, J.; Song, R.; Chen, Q.; Wroblowski, O.; et al. A highly miniaturized satellite payload based on a spatial heterodyne spectrometer for atmospheric temperature measurements in the mesosphere and lower thermosphere. Atmos. Meas. Tech. 2018, 11, 3861-3870. [CrossRef]

116. Yi, Y.; Zhang, S.; Liu, F.; Zhang, Y.; Yi, F. Laboratory fabrication of monolithic interferometers for one and two-dimensional spatial heterodyne spectrometers. Opt. Express 2017, 25, 29121-29134. [CrossRef]

117. Austin, M.D.; Ge, H.; Wei, W.; Li, M.; Yu, Z.; Wassermann, D.; Lyon, S.A.; Chou, S.Y. Fabrication of 5 nm linewidth and $14 \mathrm{n} m$ pitch features by nanoimprint lithography. Appl. Phys. Lett. 2004, 84, 5299-5301. [CrossRef] 
118. Hua, F.; Sun, Y.; Gaur, A.; Meitl, M.A.; Bilhaut, L.; Rotkina, L.; Wang, J.; Geil, P.; Shim, M.; Rogers, J.A.; et al. Polymer imprint lithography with molecular-scale resolution. Nano Lett. 2004, 4, 2467-2471. [CrossRef]

119. Yasuda, M.; Hirai, Y. Molecular Dynamics Simulations of Local Polymer Flow. In Program and Abstracts, Proceedings of the 9th International Nanoimprint \& Nanoprint Technology Conference, Copenhagen, Denmark, 13-15 October 2010; NIL Technology: Lyngby, Denmark; Obducat: Lund, Sweden, 2010; p. 32.

120. Taga, A.; Yasuda, M.; Kawata, H.; Hirai, Y. Impact of molecular size on resist filling process in nanoimprint lithography: Molecular dynamics study. J. Vac. Sci. Technol. B 2010, 28, C6M68. [CrossRef]

121. Wallrabe, U.; Salle, V. LIGA Technology for R\&D and Industrial Applications. In MEMS: A Practical Guide to Design, Analysis, and Applications; Korvink, J.G., Paul, O., Eds.; Springer: Berlin/Heidelberg, Germany, 2006; pp. 853-899. ISBN 978-3-540-21117-4.

122. Müller, C.; Mohr, L. Microspectrometer Fabricated by the Liga Process. Microsyst. Technol. 1993, 18, 273-279. [CrossRef]

123. Gibbon, M. Technologies for Photonic Integration. Ph.D. Thesis, University of Bath, Bath, UK, 1995. Available online: https: / / ethos.bl.uk/OrderDetails.do?uin=uk.bl.ethos.760668 (accessed on 13 August 2021).

124. Julijan, $\mathrm{C} . \mathrm{SiO}_{\mathrm{x}}-\mathrm{SiC}_{\mathrm{z}}$ MEMS-DBR-Based Tunable Optical Devices. Ph.D. Thesis, University of Darmstadt, Darmstadt, Germany, 2020. [CrossRef] 